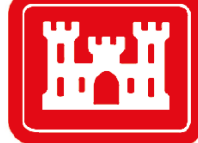

US Army Corps of Engineers ${ }_{\circledast}$

Engineer Research and

Development Center

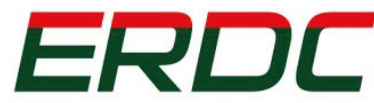

INNOVATIVE SOLUTIONS for a safer, better world

Coastal Inlets Research Program

Wave Characteristics and Sediment Resuspension by Recreational Vessels in Coastal Plain Saltmarshes

Richard Styles and Michael A. Hartman

May 2018

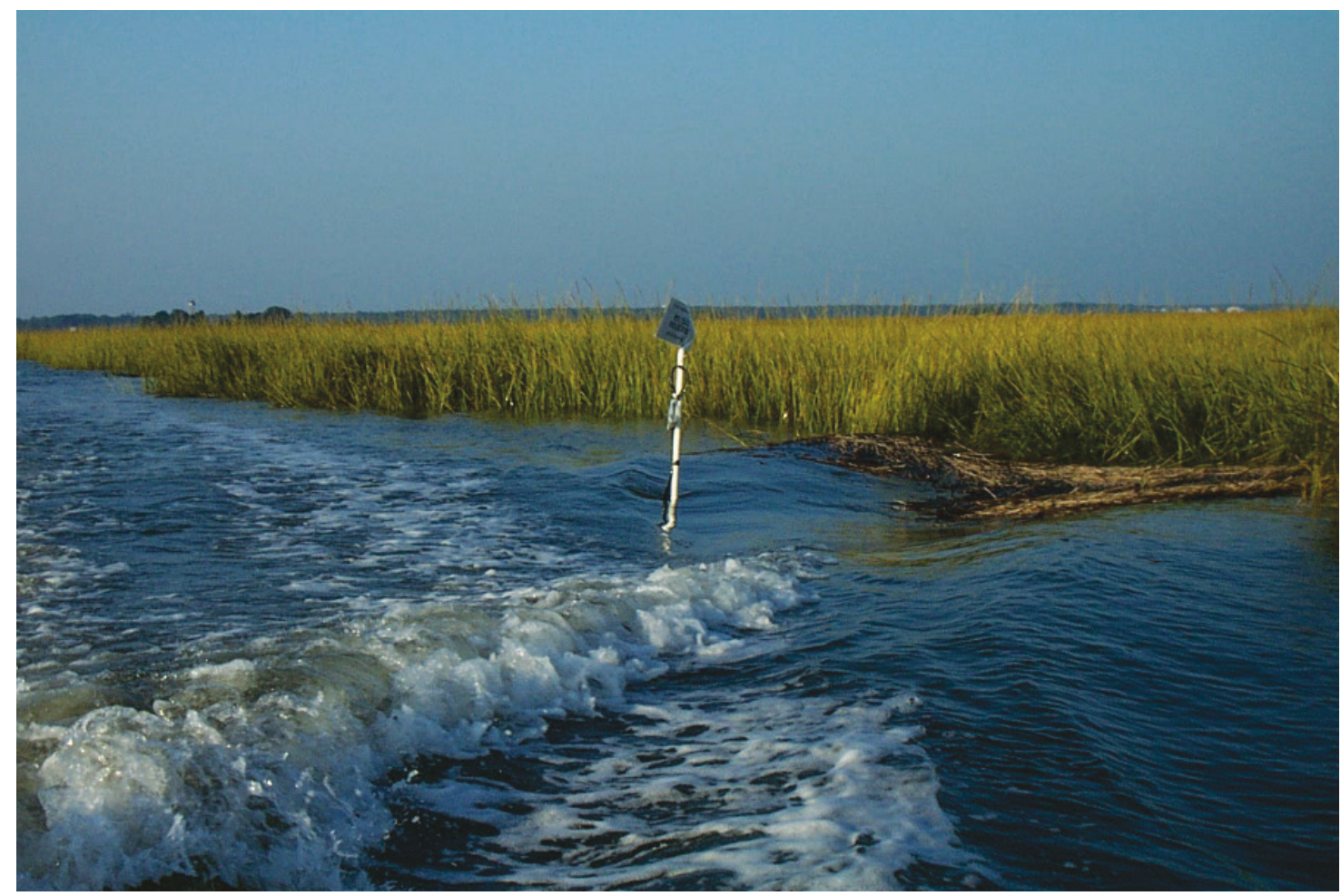


The U.S. Army Engineer Research and Development Center (ERDC) solves the nation's toughest engineering and environmental challenges. ERDC develops innovative solutions in civil and military engineering, geospatial sciences, water resources, and environmental sciences for the Army, the Department of Defense, civilian agencies, and our nation's public good. Find out more at www.erdc.usace.army.mil.

To search for other technical reports published by ERDC, visit the ERDC online library at http://acwc.sdp.sirsi.net/client/default. 


\section{Wave Characteristics and Sediment Resuspension by Recreational Vessels in Coastal Plain Saltmarshes}

Richard Styles and Michael A. Hartman

Coastal and Hydraulics Laboratory

U.S. Army Engineer Research and Development Center

3909 Halls Ferry Road

Vicksburg, MS 39180-6199

Final report

Approved for public release; distribution is unlimited.

Prepared for U.S. Army Corps of Engineers

Washington, DC 20314-1000

Under Project L85290, "Inlet Engineering Toolbox" 


\section{Abstract}

The characteristics of vessel-generated waves can form an integral component of navigation studies in coastal and inland waterways. In vulnerable areas, such as coastal wetlands, vessel wake can be a primary cause of shoreline erosion. While a few studies have investigated the role of commercial vessels operating in navigation channels adjacent to coastal marshes, little has been done regarding the role of recreational craft operating within the marsh interior. To gain further insight into the characteristics of vessel-generated waves, this study measured flow, turbulence, wave-generated currents, suspended sediment concentration, and particle size distribution in an inter-tidal salt marsh with significant tidal influence. The results are used to develop empirical equations of sediment concentration and settling velocity and to apply these equations to an existing vessel wake model. The utility of the model is demonstrated by calculating the energy dissipation due to vessels and comparing the result to the equivalent tidal energy. The cumulative energy dissipation due to vessels reveals how this information can be used to help manage recreational vessel traffic in tide-dominated coastal plain saltmarshes and extend the results of this study to a wider range of forcing conditions.

DISCLAIMER: The contents of this report are not to be used for advertising, publication, or promotional purposes. Citation of trade names does not constitute an official endorsement or approval of the use of such commercial products. All product names and trademarks cited are the property of their respective owners. The findings of this report are not to be construed as an official Department of the Army position unless so designated by other authorized documents. 


\section{Contents}
Abstract ii
Figures and Tables..

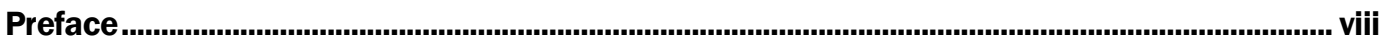

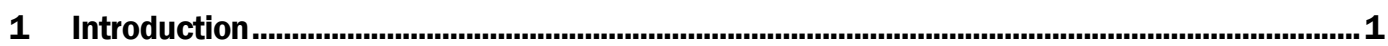

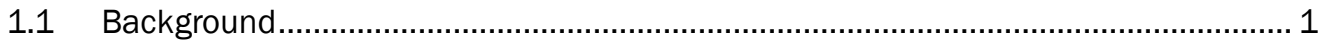

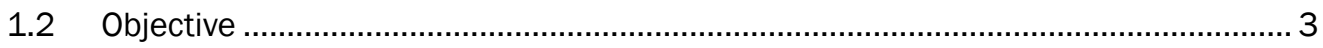

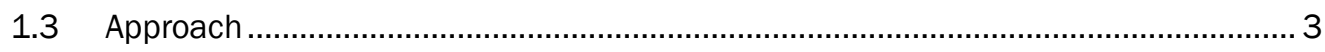

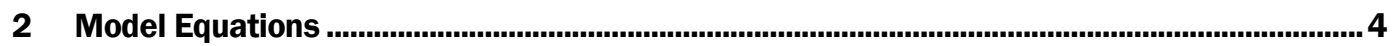

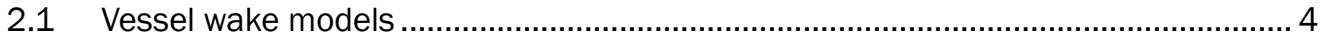

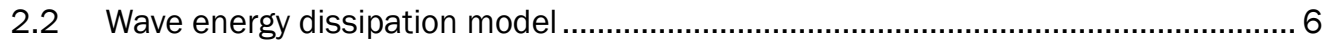

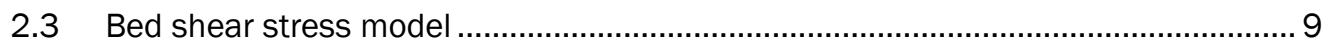

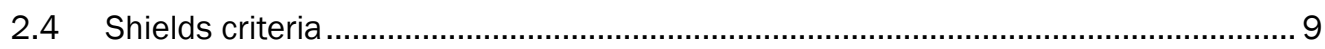

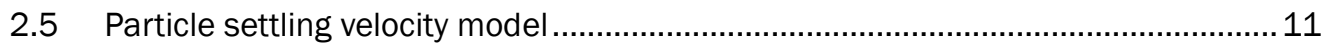

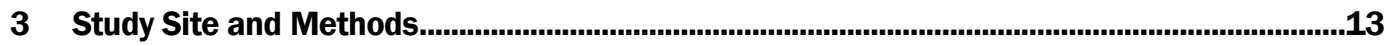

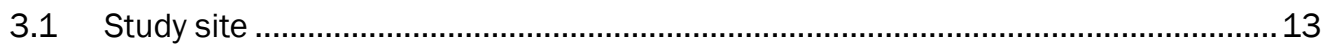

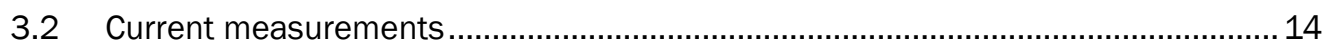

3.3 Particle size distribution measurements ……................................................... 15

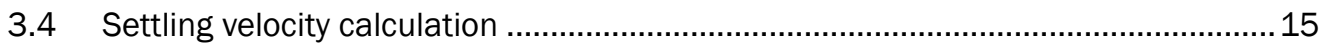

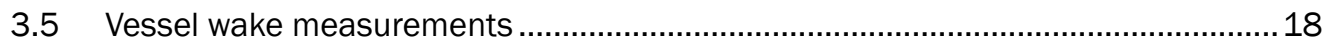

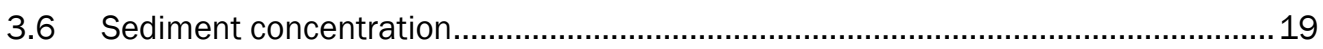

3.7 Sediment analysis ........................................................................................ 19

3.8 Reynolds stress, turbulence kinetic energy (TKE) production, and

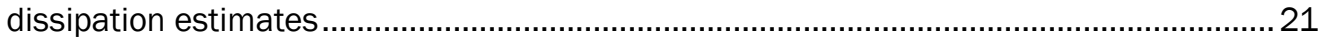

3.9 Shear parameter and Kolmogorov micro-scale ......................................................2

3.10 Wavelet analysis ........................................................................................... 23

4 Hydrodynamics and Sediment Concentration Measurements............................................24

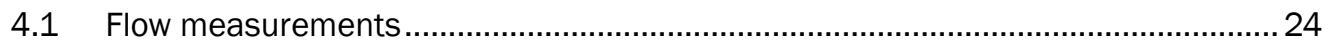

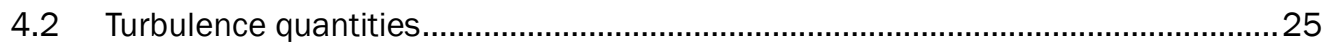

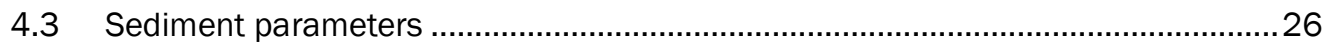

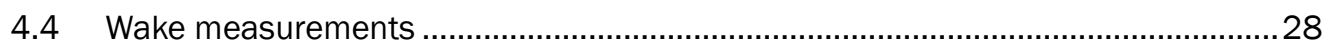

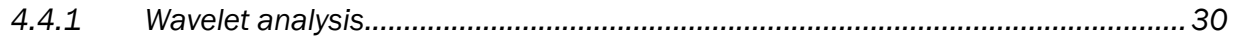

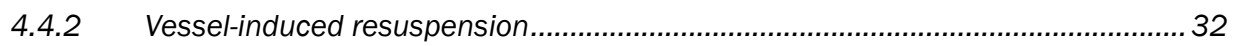

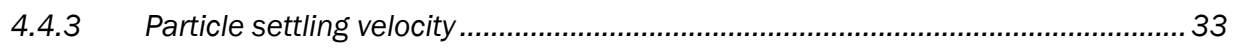

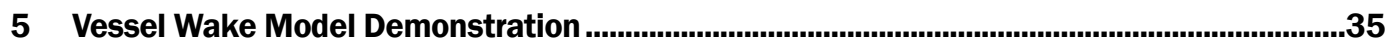

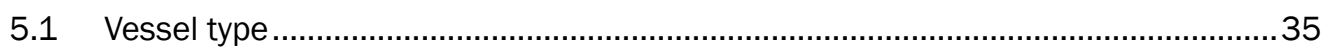

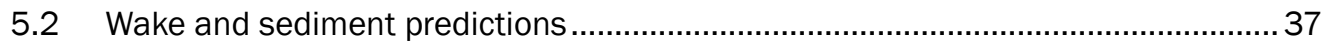

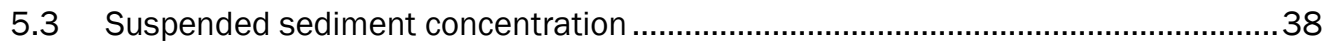

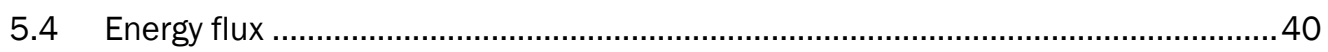




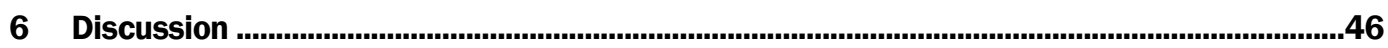

6.1 Water level in an intertidal marsh ....................................................................46

6.2 Sedimentation in oscillatory flows................................................................. 47

6.3 Settling velocity................................................................................................. 49

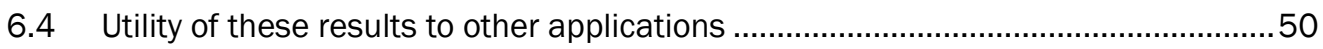

6.5 Vessel wake demonstration analysis .................................................................. 51

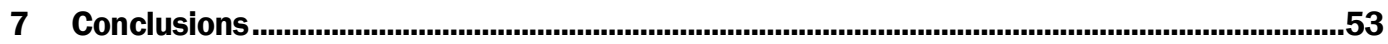

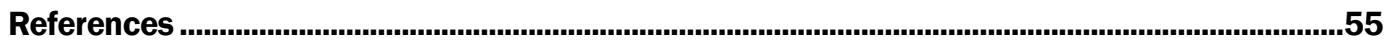

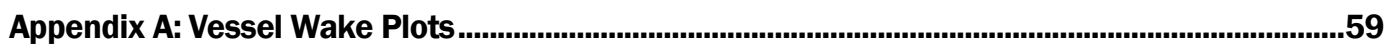

Appendix B: Vessel Wake Parameters........................................................................................69

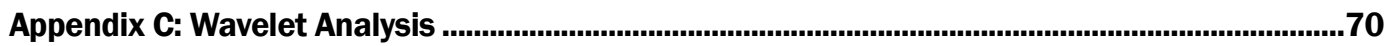

\section{Report Documentation Page}




\section{Figures and Tables}

\section{Figures}

Figure 3.1. Study site including regional coastline. The first location (Oyster) includes extensive oyster coverage, and the second (Mud) consists of a mixture of mostly silt and clay.

Figure 3.2. Instrument package deployed over the oyster bank near low tide. The instruments face the channel so that incoming waves impinge on the sampling volume before propagating over the frame. Guy-wires are used to steady the horizontal arms that hold the sensors and minimize high-frequency motions that may affect the turbulence measurements. N246 is annotated with blue tape and N230 with yellow. The yellow cylinders contain the batteries and electronics for the ADVs.

Figure 3.3. Vessel wake impinging on the saltmarsh at mid-tide when the bank is submerged. The white marker denotes the location of the instrument frame. The ADVs are approximately $0.5 \mathrm{~m}$ below the water surface.

Figure 3.4. Calibration of acoustic backscatter and concentration measurements for instrument N246. The instrument was $0.22 \mathrm{~m}$ above the bottom.

Figure 3.5. Calibration of acoustic backscatter and concentration measurements for instrument N230. The instrument was $0.40 \mathrm{~m}$ above the bottom

Figure 4.1. Flow measurements. The height above the bed (hab) denotes the distance from the bed to the ADV sampling volumes (N246 $=22 \mathrm{~cm}, \mathrm{~N} 230=40 \mathrm{~cm}$ ). Positive values denote flood currents and concentration time series is derived from N246.

Figure 4.2. Time series of turbulence parameters. The kinematic shear stress increases closer to the bed and increases with flow strength

Figure 4.3. Time series depicting sediment parameters. Particle size denotes the theoretical maximum floc size based on the Kolmogorov micro-scale and the LISST particle size associated with the highest concentration. Shear parameter is a measure of the level of turbulence needed to break up flocs.

Figure 4.4. Regression analysis of suspended sediment concentration as a function of the kinematic shear stress. The variables represent the average conditions for each burst and thus reflect the effect of the tidal currents and not vessel wake. Solid line denotes best fit. Dashed lines denote an estimate of the standard deviation of the error in predicting a future observation.

Figure 4.5. Time series of cross-shore velocity and sediment concentration for a 15-min burst that included two vessel passage events (denoted by blue arrows). The vertical axis is in the correct units for concentration and speed. The increase in velocity fluctuations clearly identify vessel wake. Concentrations increase after each vessel passage indicating wake-induced sediment resuspension.

Figure 4.6. Wavelet analysis for a burst that included two vessel passages. Time series of high-pass filtered cross-shore velocity helps to identify vessel passage events.

Figure 4.7. Wavelet analysis illustrating a single-vessel passage event. The upper panel depicts the spectral decomposition of the velocity time series. A major feature is the shift from longer to shorter periods as the wave train passes. The lower panel is the original cross-shore velocity time series.

Figure 4.8. Regression analysis of sediment resuspension due to maximum shear stress created by vessel wake. Solid line denotes best fit based on the linear regression model. Dashed lines denote an estimate of the standard deviation of the error in predicting a future observation 
Figure 4.9. Settling velocity derived from the LISST and empirical models.

Figure 5.1. Hypothetical vessel speed distribution over a tidal cycle. The tidal range is $1 \mathrm{~m}$ with a mean of $4 \mathrm{~m}$ depth at the location of the vessel. The plot represents 80 vessel passages at different phases of the tide to emulate possible conditions for a coastal plain saltmarsh similar to North Inlet

Figure 5.2. Wave height and kinematic shear stress distribution over a tidal cycle.

Figure 5.3. Suspended sediment concentration predictions. The results represent the suspended sediment concentration measured at $0.22 \mathrm{~cm}$ above the bed......

Figure 5.4. Distribution of concentration due to tidal flow computed from the ADV acoustic backscatter.

Figure 5.5. Energy dissipated over a tidal cycle assuming eight waves per wave packet. The horizontal dashed line is the tidally averaged energy dissipation. Error bars denote \pm one standard deviation.

Figure 5.6. Energy dissipation over a tidal cycle for 10 waves per wave group. The horizontal dashed line is the tidally averaged energy dissipation.

Figure A.1. First burst in which two vessel wake events occurred....................................................60

Figure A.2. Second burst with four vessel wake events........................................................................61

Figure A.3. Third burst with three vessel wake events....................................................................62

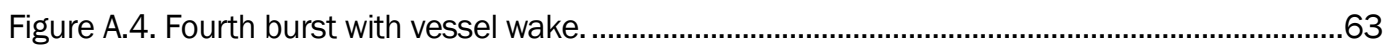

Figure A.5. Fifth burst with vessel wake.........................................................................................64

Figure A.6. Sixth burst with vessel wake and the last burst over the oysters. ...................................65

Figure A.7. Seventh burst with vessel wake. This is the first burst over the mud bank...................66

Figure A.8. Eighth burst with vessel wake.......................................................................................67

Figure A.9. Final burst with vessel wake. .....................................................................................68

Figure C.1. Wavelet analysis for the first burst with vessels.............................................................70

Figure C.2. Wavelet analysis for the second burst with waves. The second wave event indicates the largest shift in period.

Figure C.3. Wavelet analysis for third burst with waves. The mean along channel current is the highest for the first two tidal cycles. The increase in energy density in the 4 to $16 \mathrm{~s}$ band is due to the added turbulence.

Figure C.4. Wavelet analysis for the fourth burst with waves. The mean along channel current is reduced, and there is a corresponding reduction in the energy density except for the vessel wake.

Figure C.5. Wavelet analysis for the fifth burst with waves. The multiple vessel wake events leads to residual energy bands in the 8 to $16 \mathrm{~s}$ range that persist after the last wake has passed. Maximum orbital velocities are relatively low $(5 \mathrm{~cm} / \mathrm{s})$, so the energy from waves is small.

Figure C.6. Wavelet analysis for the sixth burst with waves. Maximum orbital velocities are relatively large. There are residual energy pockets at different frequencies that form in the wake of the last vessel passage. However, their energy is small compared to the vessel waves.

Figure C.7. Wavelet analysis for seventh burst with vessels. The energy density is narrow banded and confined to a small temporal window. There is very little residual energy pulse following the vessel wake event.

Figure C.8. Wavelet analysis for the eighth burst with vessels. Energy density is narrow banded with some residual energy pockets following the wake event. 
Figure C.9. Wavelet analysis for the ninth burst with vessels. This is the last burst with vessels. Mean along channel currents are the highest measured for the study, which is reflected in the wavelet analysis as lower-frequency energy density pockets and larger fluctuations in the time series.

\section{Tables}

Table 5.1. Vessel characteristics for $21 \mathrm{ft}$ Boston Whaler Outrage used as input to the Maynord (2005) wake model.

Table B.1. Measured vessel wake parameters. 


\section{Preface}

This study was sponsored by the Coastal Inlets Research Program (CIRP), under the "Inlet Engineering Toolbox" work unit. The CIRP is funded by the Operation and Maintenance (O\&M) Navigation Business Line of the Headquarters, U.S. Army Corps of Engineers (HQUSACE), and is administered by the U.S. Army Engineer Research and Development Center (ERDC), Coastal and Hydraulics Laboratory (CHL), Vicksburg, MS, under the Navigation Program of HQUSACE.

Mr. Jeffrey A. McKee is HQUSACE Navigation Business Line Manager overseeing the CIRP, and Mr. W. Jeff Lillycrop, CHL, is the ERDC Technical Director for Civil Works and Navigation, Research, Development, and Technology Transfer Portfolio. This study was conducted by the Coastal Engineering Branch (CEERD-HNC) of the Navigation Division (CEERD-HN) of CHL. At the time of publication of this report, oversight and guidance of this work was provided by Mr. Gregory Dreaper, Acting Chief, CEERD-HNC; Dr. Jackie S. Pettway, Chief, CEERD-HN; and Ms. Mary Cialone, CIRP Program Manager.

Mr. Jeffrey R. Eckstein was Acting Director of CHL.

COL Bryan S. Green was Commander of ERDC, and Dr. David W. Pittman was Director. 


\section{Introduction}

Vessel-generated waves in channels impact the shoreline and habitat structure in many waterways (Kurennoy et al. 2009; Osborne and Boak 1999). From large commercial vessels operating in managed navigation channels to recreational craft cruising in reservoirs, lakes, and other confined water bodies, the energy dissipated due to wave breaking can affect shoreline stability and reduce water quality (Asplund 2000). The potential for shoreline erosion scales with wake height and vessel activity; increases in vessel passages per unit time equate to a greater number of waves reaching the shoreline. Therefore, understanding vessel wake characteristics such as frequency of occurrence, wave amplitude, and wave dispersion are primary factors when developing tools to assess the environmental impacts of vessel traffic.

In regions with primarily fine-grained sediments, the resuspended material settles slowly so that the water column maintains higher particle concentrations for longer time periods. Locally, light attenuation through scattering and absorption can reduce water quality (Bilotta and Brazier 2008). If the material is carried by the ambient current, it may settle in other regions, posing ecological risks to benthic habitats (Asplund 2000; Houser 2011). Higher concentrations are likely to produce thicker marine deposits increasing the chance of burial and posing additional risk to benthic communities.

The Civil Works program of the U.S. Army Corps of Engineers (USACE) is responsible for managing many navigation, flood risk management, and environmental projects that are impacted by vessel wake. The purpose of this report is to present a simple vessel wake tool that predicts bottom stress and associated sediment resuspension due to vessel waves in coastal and riverine settings. The methodologies, calibrations, and demonstrations presented in this report can be applied to projects with a need to understand the role of wake-induced erosion and water quality in tide-dominated coastal plain saltmarshes and similar water bodies.

\subsection{Background}

There is vast literature on the impacts of vessel wake in the environment including the work of Houser (2010); Kurennoy et al. (2009); Maynord 
(1996); PIANC (2003); and Sorensen (1997) among many others. The literature can generally be divided in terms of the environmental conditions and navigation use practices. Environmental divisions include the type, size, and depth of waterbodies such as rivers, lakes, reservoirs, estuaries, bays, coasts, and channels. The different settings have vastly different physical characteristics that influence the degree to which vessel wake can impact the system including distance between the sailing line and shore, bank and bed type (gravel, mud, sand, armored), tides, ambient wind, wave and current conditions, and bathymetry. The likelihood and degree of bank erosion is highly dependent on bank type (Maynord et al. 2008), and the distribution of benthic habitats including oysters, submerged aquatic vegetation, etc., with respect to regions of major vessel activity is important in determining the degree to which these areas are vulnerable to vessel waves.

Navigation usage comprises recreational and commercial activities. Commercial craft include large seafaring vessels such as cargo ships and tankers as well as smaller craft such as work boats, motor vessels, barges, pilot boats, and ferries. In addition to divergent wakes, commercial vessels generate transverse stern waves (drawdown), which are several times larger than bow waves (Maynord 1996). Commercial vessels also operate in confined areas with a somewhat predictable traffic pattern. As such, the cumulative effect of commercial vessels is more important than a single isolated vessel. Recreational craft include skiffs, cabin cruisers, jet skis, pontoon boats, and a wide variety of other small motorized craft.

Recreational craft are generally lighter and operate at higher average speeds than their commercial counterparts. As such, recreational vessels can travel closer to the shoreline and within shallow water areas that are more pristine and vulnerable to the effects of wake.

While a large body of literature exists on the effects of ship wake, fewer studies have focused on saltmarshes in particular (Cox et al. 2003; Houser 2010, 2011). Of these, the majority concentrate on commercial vessels operating near fringing saltmarshes where the primary wake source is large commercial vessels including container ships and tankers. These vessels generate the largest wakes, especially the drawdown, which can produce large (0.5 - 1 meter $[\mathrm{m}])$ fluctuations in water level that dominate the wave signal and associated wave energy flux (Maynord 2007). The degree to which recreational craft contribute to the total wave energy flux has not been studied in saltmarshes. However, Maynord et al. (2008) 
investigated the role of recreational vessels operating along the Kenai River in Alaska. The considerable sports fishing industry in this area leads to very high concentrations of boat traffic, especially during holiday weekends. The cumulative effect of vessels was estimated to contribute up to $16 \%$ above the total river energy, which would significantly contribute to shoreline erosion.

Recreational vessels travel at high speeds and can operate within a few meters of the shoreline whereas commercial vessels are confined to navigation channels. Recreational craft can penetrate the marsh interior where vessel wake originating from the navigation channel may not reach. Even though recreational craft may not generate waves as large as commercial vessels, they directly affect more vulnerable regions of the wetland interior that otherwise do not experience any impact from commercial traffic operating in navigation channels.

\subsection{Objective}

The focus of this technical report is to examine the effect of vessel wake on sediment dynamics in a coastal plain saltmarsh. Measurements of waves, currents, and suspended sediment concentration are used to investigate sediment transport formulas that relate the orbital velocities caused by vessel wake to the local suspended sediment concentration. A simple wake model is coupled to a bed stress model to predict wave height and energy dissipation. In this way, it is possible to compare energy dissipation due to vessel wake to the ambient tidal forcing, which is used to estimate the relative impact of vessel traffic on shoreline erosion.

\subsection{Approach}

The modeling approach uses simple yet established theories of vesselgenerated waves that can be easily applied to other areas with similar environmental and vessel traffic conditions. This report is organized as follows. Chapter 1 introduces the study. Chapter 2 presents the vessel wake, wave, and sediment mobility equations. Chapter 3 describes the study site, methods, and sediment concentration analysis. Chapter 4 presents the sediment concentration and hydrodynamic measurements. Chapter 5 presents an application of the vessel wake tool in an intertidal salt marsh environment. Chapter 6 presents discussion focusing on model performance and limitations for applications in real environments. Chapter 7 presents the conclusions. 


\section{Model Equations}

Given that the process of wake generation is highly nonlinear, mathematical models have been developed using empirical approaches. The equations governing vessel wake generation and propagation are site specific, and extrapolation to other environments and vessel types requires calibration and verification to assess model accuracy. As such, a number of wake models have been developed using a variety of data sources for different types of ships. Models for small recreational craft are generally based on different underlying assumptions than models designed for commercial vessels (Sorensen 1997). This leads to different equations and empirical coefficients that are specific to vessel type. This does, however, allow the generalization of vessel wake models for use in different settings as long as the underlying assumptions used to create the model are not violated (i.e., models developed for recreational craft should only be used to model wake generated by recreational craft.)

\subsection{Vessel wake models}

A wide variety of mathematical models have been developed to describe wake generation from moving vessels (Sorensen 1997). All approaches rely on empirically derived relationships to express wave height as a function of speed and in some instances other geometric characteristics of the vessels such as draft, beam, vessel length, and displaced vessel weight or volume. Some models are designed to predict the maximum wave height associated with the vessel while others also predict the decay in wave height away from the vessel. For applications to predict shoreline erosion, the predictive model must account for the decay in wave height with distance from the vessel.

An important parameter in the study of vessel wake is the vessel Froude number,

$$
F r=\frac{V_{s}}{\sqrt{g h}}
$$

where:

$$
\begin{aligned}
V_{s} & =\text { vessel speed } \\
g & =\text { acceleration due to gravity } \\
h & =\text { water depth. }
\end{aligned}
$$


For most commercial vessels operating in shallow water, $F r$ is less than 1 , which is termed subcritical flow. For high-speed recreational craft, $F r$ can exceed unity, which is termed supercritical flow. The wake characteristics are different depending upon whether $F r$ is less than or greater than 1 . The variation in wake patterns due to different vessel speeds are beyond the scope of this report, and a description of vessel wake transition is provided in Soomere (2007).

Based on a review of existing vessel-generated wave height data and further analysis and calibration of empirical coefficients, Maynord (2005) developed the following vessel wake equation for recreational craft:

$$
\frac{H_{m}}{W^{1 / 3}}=C_{m} F_{w}^{-0.58}\left(\frac{x}{W^{1 / 3}}\right)^{-0.42}
$$

where:

$$
\begin{aligned}
\mathrm{H}_{\mathrm{m}}= & \text { is wave height predicted from the Maynord (2005) model } \\
\mathrm{W}= & \text { volume displacement of the vessel = total vessel weight/unit } \\
& \text { weight of water } \\
x= & \text { distance from vessel centerline } \\
C= & \text { vessel shape coefficient that is equal to } 1.0 \text { for v-hull and } 0.82 \\
& \text { for flat bottom. }
\end{aligned}
$$

The displacement Froude number is defined as

$$
F_{W}=\frac{V_{s}}{\sqrt{g W^{1 / 3}}}
$$

Maynord's model is for vessels that typically operate over a large range of speeds and are generally smaller and lighter than commercial vessels. For a given volume displacement and hull shape, the equation is a function only of vessel speed. Maynord (2005) identifies three modes of vessel planing, each with distinct wake height characteristics that help define the operational conditions for the model. Displacement mode vessels (slow speed), in which the dominant force on the hull is the hydrostatic lift, produce wave heights that increase with increasing speed. Semi-planing vessels (medium speed), in which the lift force is shared between the hydrostatic and hydrodynamic lift, causes the vessel to partially rise out of the water. Semi-planing vessels produce the highest waves, and the 
relationship between speed and wave height undergoes a transition as the vessel speed increases. At lower speeds within the semi-planing mode, wave height increases with increasing vessel speed. At higher speeds, wave height decreases with increasing speed. The transition is related to the vessel dynamics as the lift force shifts from being primarily hydrostatic to being primarily hydrodynamic. The latter of which is caused by the increase in dynamic pressure, which lifts the boat higher out of the water decreasing vessel displacement and reducing the maximum wake height. Fully planing vessels (high speed), in which the dominant force on the hull is the hydrodynamic lift, produce waves that decrease in height with increasing speed. The Maynord (2005) model is only applicable to semiplaning and fully planing vessels as the equation predicts a decrease in wave height with increasing vessel speed.

The minimum vessel speed used to calibrate the Maynord (2005) model is 3 meters per second $(\mathrm{m} / \mathrm{s})$ (6 knots). As such, this model is not recommended for recreational vessels with speeds $<3 \mathrm{~m} / \mathrm{s}$, due to a lack of calibration data for the empirical equations. In addition to speed, the other parameter used in the model is the relative vessel weight, which can be expressed as the mass of the vessel divided by the density of water, or the weight of the vessel divided by the unit weight of water.

\subsection{Wave energy dissipation model}

As vessel waves approach the shore, they begin to shoal, growing in height until exceeding the critical steepness and then break. Breaking waves release the stored potential and kinetic energy of the wave, which can resuspend sediment and contribute to bank erosion.

The total energy per unit planform area $(E)$ for a monochromatic linear wave is written as

$$
E=\frac{1}{8} \rho g H^{2}
$$

where:

$$
\begin{aligned}
& \rho=\text { fluid density } \\
& H=\text { wave height. }
\end{aligned}
$$


The wave energy flux represents the horizontal propagation of wave energy and is written as

$$
E_{f}=E C_{g}
$$

The last term is the wave group speed $\left(C_{g}\right)$, and it is related to the phase speed $(C)$ by

$$
C_{g}=\frac{1}{2} C\left(1+\frac{2 k h}{\sinh 2 k h}\right)
$$

where:

$$
\begin{aligned}
& C=L / T \\
& L=\text { wavelength } \\
& T=\text { wave period } \\
& k=\text { wave number }=2 \pi / L .
\end{aligned}
$$

The energy flux measures the energy dissipated when the wave breaks. In applications, the energy due to vessel waves is routinely gauged in terms of the ambient energy associated with flow processes for a given situation (Maynord 2008). For rivers, this is the stream flow energy, and for coastal settings it can be tidal or wind driven flow or the wind-generated wave energy.

The phase speed for a steadily moving vessel can be written as

$$
C=V_{s} \cos \theta
$$

where $\theta$ is the angle between the sailing line and the direction of wave propagation (Sorensen 1997). The angle $\theta$ is theoretically determined to be equal to 35.27 degrees for deep water waves (Thompson 1887). Under these conditions

$$
C=0.816 V_{s}
$$

The angle $\theta$ varies between 35.27 degrees for deep water waves to $\mathrm{o}$ as $F_{r}$ approaches 1. Weggel and Sorensen (1986) developed the following empirical expression using data from existing sources: 


$$
\theta=35.27\left(1-e^{12\left(F_{r}-1\right)}\right) \quad F_{r}<1
$$

If $F_{r}>1$, the wave angle formed at the bow becomes a straight divergent wave with an angle to the sailing line given by

$$
\theta=90^{\circ}-\arcsin \left(\frac{1}{F_{r}}\right) \quad F_{r}>1
$$

Equation (9) or (10) can be substituted into Equation (7) to define C. A modified form of the dispersion relationship can then be used to determine the wavenumber:

$$
C^{2}=\frac{g}{k} \tanh (k h)
$$

The wavenumber can be used to determine $L$, which is then used to compute the wave period as $T=L / C$. Once $T$ is known, then the dispersion relationship can be used to define the wave transformation away from the vessel as it travels into shallower water towards the shoreline.

Given the wave height, wavenumber, and water depth, linear wave theory is used to compute the maximum bottom wave orbital velocity $\left(U_{b}\right)$ as

$$
U_{b}=\frac{\omega H}{2 \sinh (k h)}
$$

and the associated bottom excursion amplitude, $A_{b}=U_{b} / \omega$, where $\omega=$ $2 \pi / T$. The maximum wave orbital velocity will vary with distance from the vessel and water depth. Wave height decays with distance from the vessel due to diffusion of energy along the crest, leading to a corresponding reduction in $U_{b}$. However, water depth decreases as the wave propagates into shallower water, eventually breaking at the shoreline. For given wave characteristics, bottom orbital velocity increases with decreasing water depth. The point where decreases in wave height balance decreases in water depth in such a way as to keep $U_{b}$ constant can be evaluated by setting the right-hand side of Equation (12) equal to a constant. This curve uniquely defines $H$ in terms of $h$ for a given bottom orbital velocity. In this way, it is possible to define the unique combination of $H$ and $h$ that define the point of initiation of sediment motion. 


\subsection{Bed shear stress model}

The instantaneous bottom stress associated with a wave $(\tau w)$ is written as

$$
\tau_{w}=1 / 2 \rho f_{w} U_{w b}^{2}
$$

where:

$$
\begin{aligned}
f_{w} & =\text { wave friction factor } \\
U_{w b} & =\text { instantaneous bottom velocity for the wave. }
\end{aligned}
$$

When the wave stress exceeds the minimum for the initiation of sediment motion, sediment is mobilized, and the bed may begin to erode. In practice, the maximum bottom stress $\left(\tau_{w m}\right)$ over a wave cycle is commonly used in sediment transport models. Modeling the concentration and associated bed erosion depends on the types of sediment present in the bed, and different modeling approaches are used for consolidated versus unconsolidated sediments.

\subsection{Shields criteria}

Initiation of sediment motion for non-cohesive sediments is determined using the Shields criteria,

$$
\psi_{c r}=\frac{\left|\tau_{b c r}^{\prime}\right|}{\rho(s-1) g d}
$$

where:

$$
\begin{aligned}
\left|\tau_{b c r}^{\prime}\right|= & \text { the magnitude of the skin friction shear stress for the initiation } \\
& \quad \text { of sediment motion } \\
s= & \text { the relative sediment density }\left(=\rho_{s} / \rho\right) \\
\rho_{s}= & \text { sediment density } \\
d= & \text { the representative grain diameter. }
\end{aligned}
$$

The critical Shields parameter for the initiation of sediment motion $\left(\psi_{c r}\right)$ is obtained using empirical equations given the sediment grain size. The Shields parameter representing the wake-induced stress acting on the bed at any moment is 


$$
\psi_{w m}=\frac{\left|\tau_{w m}^{\prime}\right|}{\rho(s-1) g d}
$$

where $\left|\tau_{w m}^{\prime}\right|$ is the maximum wave shear stress based on skin roughness. Sediment is mobilized when $\psi_{w m}>\psi_{c r}$. The friction factor and associated wave stress are computed using the bottom boundary layer model of Styles and Glenn (2000) for pure waves. The friction factor is a function of the relative roughness defined as $k_{b} / A_{b}$, where $k_{b}$ is the bed roughness (Styles and Glenn 2000). For computing sediment mobilization, $k_{b}$ is a function of the sediment grain diameter $(k b=2 d)$. (The derivation is not presented here, and the reader is referred to Styles and Glenn $(2000,2002)$ for further details.)

For consolidated sediments (clays, silts, flocs) erosion models express the concentration as a function of the excess shear stress,

$$
E=M\left(\frac{\tau_{b}-\tau_{c}}{\tau_{c}}\right) \quad \tau_{b}>\tau_{c}
$$

where:

$$
\begin{aligned}
E & =\text { erosion rate } \\
M & =\text { erosion rate parameter } \\
\tau_{b} & =\text { mean bed shear stress } \\
\tau_{c} & =\text { critical shear stress for erosion of consolidated sediment. }
\end{aligned}
$$

Under waves, $\tau_{b}$ can be replaced with $\left|\tau_{w m}^{\prime}\right|$ to define the wave-induced stress. Establishing the critical value for consolidated sediments is generally more complicated than the critical value associated with the Shields criteria, as sediment erosion is affected by additional processes including particle adhesion and bed permeability (Winterwerp and Van Kesteren 2004). For loosely consolidated deposits, erosion can occur for stresses on the order of 0.1 pascal. If the sediment is compacted, which increases the bonding strength between grains and reduces porosity, the minimum stress can be an order of magnitude higher (Winterwerp and Van Kesteren 2004). For applications, direct measurements of the bed sediment type may not be available. Under these conditions, a common approach is to describe the likelihood of resuspending the most amount of sediment as this establishes an upper bound on the potential for erosion. 
In this case, $\tau_{c}$ can be set equal to a characteristic stress value for a given type of sediment associated with a specific region. In the present study, model estimates derived from measurements are used to obtain a relationship between concentration and shear stress, so the direct estimate of the erosion rate is not necessary. Instead, the concentration is expressed as a linear function of the shear stress with the fitting coefficient obtained from the regression analysis.

\subsection{Particle settling velocity model}

In fine-grained sediment environments such as saltmarshes, flocs can account for a significant fraction of the suspended load. Because of their water content, flocs are less dense and have lower settling velocities than their solid particle counterparts. A widely reported model used to estimate particle settling is the Stokes equation (Winterwerp and Van Kesteren 2004). The Stokes theory assumes that the tendency for a particle to settle under the action of gravity is balanced by fluid drag. Since drag is proportional to velocity, the gravity term in the momentum equation balances the friction term to produce the following equation:

$$
w_{f s}=\frac{(s-1) g d^{2}}{18 v}
$$

where $v=$ the kinematic viscosity of water and $w_{f s}$ denotes settling velocity for the Stokes equation. The Stokes equation is for individual particles, but it has been used to study the behavior of flocs so long as the floc density is used to define $s$ and $d$ is floc diameter.

Other investigators have developed generalized settling velocity models based only on floc size. Based on a series of laboratory experiments in an annular flume, Manning and Dyer (1999) developed the following settling velocity model for flocs:

$$
w_{f i n}=0.0033 d^{1.137}
$$

where $d$ is in microns and the subscript denotes the Manning and Dyer model. Units for $w_{f m}$ are centimenters per second (cm/s). Gibbs (1985) reviewed field data to develop another model also based only of floc diameter: 


$$
w_{f g}=1.73 d^{0.78}
$$

where $d$ is in $\mathrm{cm}$ and $w_{f g}$ is in $\mathrm{cm} / \mathrm{s}$.

Porosity increases water content so that flocs have a lower density than their individual particle constituents. As such, the relative sediment density in the Stokes equation should be expressed in terms of the floc density rather than the density of a single grain with diameter, $d$. Manning and Dyer (1999) also developed an empirical model of floc density as a function of particle size:

$$
\rho_{f}=8785.8 d^{-0.9992}+\rho
$$

where $d$ is in microns and $\rho f$ is in kilograms per cubic meter $\left(\mathrm{kg} / \mathrm{m}^{3}\right)$. This expression shows that floc density decreases with floc size as large flocs tend to contain higher water content leading to a lower effective density compared to individual particles. This formula is used to compute the relative sediment density in Equation (17). 


\section{Study Site and Methods}

\subsection{Study site}

The study was carried out in June 2007 in the North Inlet estuary located near Georgetown, SC (Figure 3.1). North Inlet is a coastal plain salt marsh consisting of low-relief mud flats dissected by a sinuous network of sub-and intertidal channels. The system receives little freshwater input, and the hydrodynamics are dominated by tidal processes. Tidal range at the entrance is $1.5 \mathrm{~m}$, with maximum current speeds in the inlet throat that vary between 0.5 and $1.0 \mathrm{~m} / \mathrm{s}$ as a function of the fortnightly tide (Kjerfve 1986; Wargo and Styles 2007). Friction causes the tidal range and associated currents to decrease as the tide penetrates farther into the estuary.

Figure 3.1. Study site including regional coastline. The first location (Oyster) includes extensive oyster coverage, and the second (Mud) consists of a mixture of mostly silt and clay.

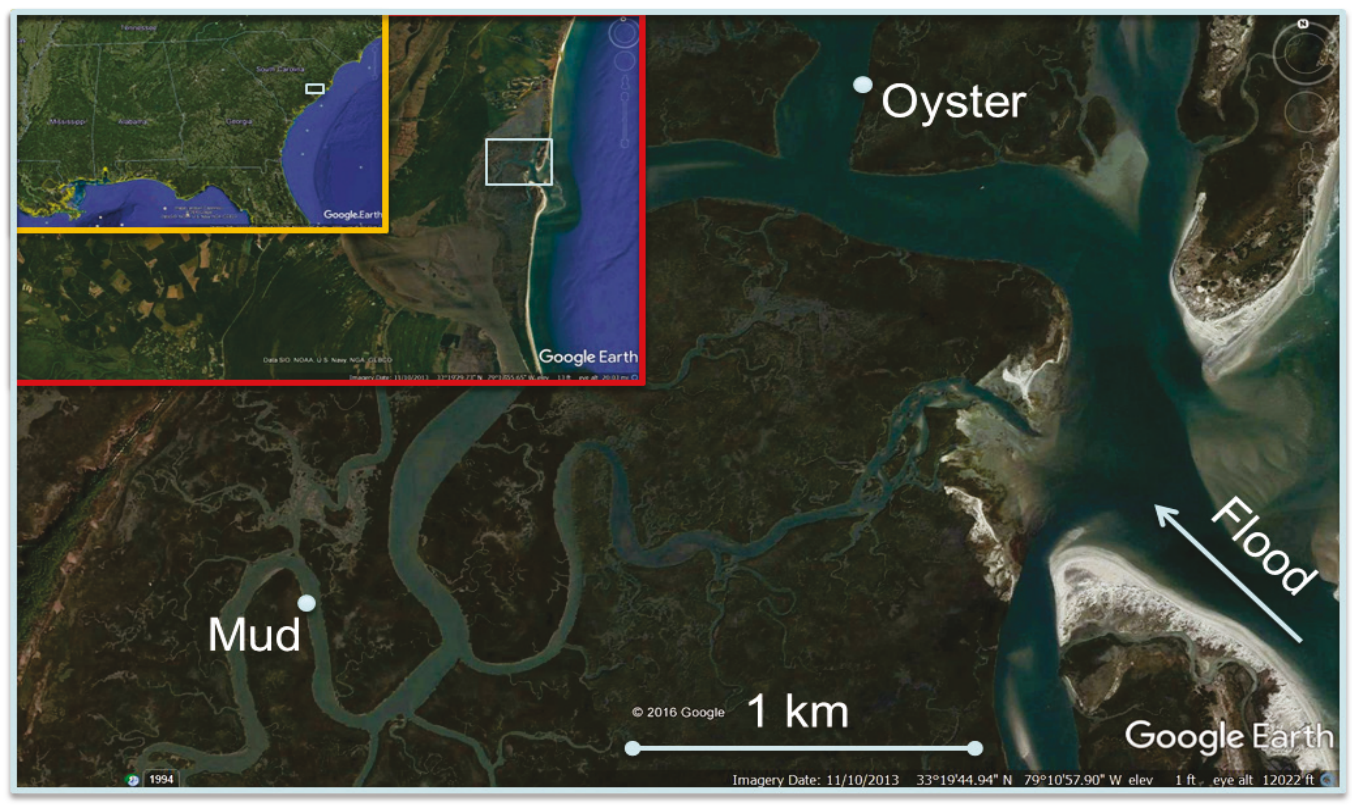

The study was carried out over a 2-day period by deploying sensors to measure flow and sediment concentration on the bank of two sub-tidal channels. The first day, the instruments were deployed in Old Man Creek over a bed of oysters. The second day, the instruments were redeployed along an oyster-free section of Town Creek farther from the inlet entrance. The second site was one, of only a very few locations within the estuary, that was oyster free. The instrument package included a pair of acoustic Doppler velocimeters (ADVs) and a laser in situ scattering transmissometer (LISST). The instruments were mounted on two separate frames and deployed near low tide (Figure 3.2). 
Figure 3.2. Instrument package deployed over the oyster bank near low tide. The instruments face the channel so that incoming waves impinge on the sampling volume before propagating over the frame. Guy-wires are used to steady the horizontal arms that hold the sensors and minimize high-frequency motions that may affect the turbulence measurements. N246 is annotated with blue tape and N230 with yellow. The yellow cylinders contain the batteries and electronics for the ADVs.

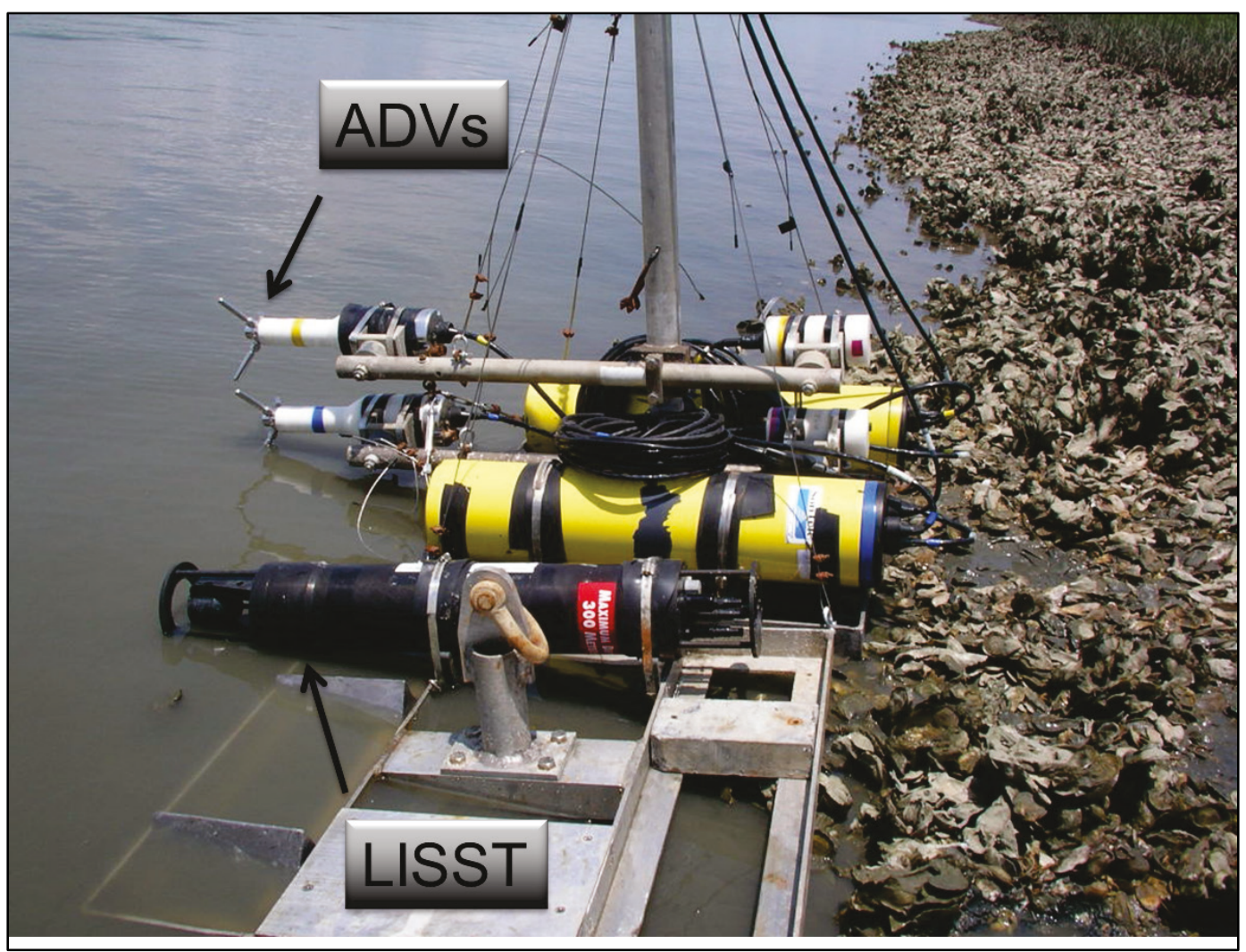

\subsection{Current measurements}

Direct measurements of flow were acquired using a pair of Sontek ADVs. The sensors were deployed on a single frame and vertically stacked, with the lower sensor (denoted N246, blue tape in Figure 3.2) positioned $22 \mathrm{~cm}$ above the bed and the upper sensor (denoted N230, yellow tape in Figure 3.2) positioned $40 \mathrm{~cm}$ above the bed (Figure 3.2). The sensors were programmed to sample at 10 hertz for a 15 -minute (min) burst every $20 \mathrm{~min}$. The first day the bursts were scheduled to begin at 10,30, and 50 min past the hour. The second day the sensors were reprogrammed to start at $\mathrm{O}, 20$, and $40 \mathrm{~min}$ past the hour. Each burst produced 9,000 (10 points $/ \mathrm{s} \times 60 \mathrm{~s} / \mathrm{min} \times 15 \mathrm{~min}$ ) data points.

The velocity vector $(u, v, w)$ was divided into burst-averaged $(U, V, W)$ and fluctuating $\left(u^{\prime}, v^{\prime}, w^{\prime}\right)$ components. The coordinate system was chosen such that $u$ was cross-channel, $v$ was along channel, and $w$ was vertical. The sign 
convention for the horizontal components was positive for flood and towards the channel bank. The vertical component was positive upwards.

\subsection{Particle size distribution measurements}

A LISST was deployed adjacent to the ADV frame. The LISST is a laser diffraction instrument that measures suspended sediment concentration and size distribution based on the forward angle scattering properties of particles (Agrawal and Pottsmith 2000). A Type B LISST was used in the study, which measured suspended sediment concentration in 32 log-spaced bins ranging from 2.5 to 500 microns. The LISST was also equipped with a pressure sensor to measure water depth. The LISST sampling volume was $20 \mathrm{~cm}$ above the bed, and the pressure sensor was $25 \mathrm{~cm}$ above the bed. The sampling rate of the LISST is designed to collect approximately 64 samples every $17 \mathrm{~s}$. It takes a few seconds for the internal software to average the samples and reset the instrument and laser to collect another burst. To ensure enough time for the instrument to collect, average, and save the samples, the LISST was programmed with a $30 \mathrm{~s}$ burst. LISST accuracy is measured by the attenuation signal as measured by the beam intensity at the collector. The manufacturer states that accurate measurements are possible when the ratio of the transmitted beam to the emitted beam is $>$ $35 \%$. LISST data for which this ratio $<35 \%$ are excluded.

\subsection{Settling velocity calculation}

Sediment dynamics due to vessel wake cannot accurately be described using wave-averaged or steady flow approaches because of the finite duration of the wave packet generated by a single vessel. As the vessel passes, the waves agitate the bed and resuspend sediment if the shear stress during part of the wave cycle is greater than the critical value for erosion. If the sediment is fine-grained, it will settle slowly, and high concentrations of suspended material may persist after the vessel wake has passed. Without waves to further resuspend sediment, the only vertical force is gravity, and particles settle. This process would appear in a time-series measurement as an initial rapid increase in concentration coincident with the passing wave, followed by a steady decay until the concentration returns to background levels.

In steady or wave-averaged flows, the tendency for particle settling is balanced by the upward turbulent flux. As such, the resuspension process is more diffusive than advective. For vessel wake, the resuspension process 
consists of a rapid uplift of particles followed by a more quiescent period of gravitational settling after the wave train has passed. For vessel wake, the resuspension process is more advective than diffusive.

A simple flux model based on conservation of mass is used to estimate particle settling velocity. Assuming that the only force acting on a suspension of particles is gravitational settling, conservation of sediment mass is written as

$$
\frac{\partial C}{\partial t}=w_{f} \frac{\partial C}{\partial z}
$$

where $C$ is concentration. In this model, horizontal advection is neglected as well as any turbulent-induced vertical mixing. Particles in the water column settle out of suspension at a rate proportional to their individual settling velocity $\left(w_{f}\right)$. Equation (21) does not explicitly consider particle size distribution, but it can be applied to different grain size classes. When used in applications, it is assumed that the water column can be treated as an isolated box, allowing the vertical gradient term to be discretized. Assuming that there is no sediment flux across the water surface, the vertical sediment flux passing through a horizontal plane at a depth $h_{c}$ allows (21) to be written as

$$
\frac{1}{C\left(h_{c}\right)} \frac{\partial C\left(h_{c}\right)}{\partial t}=\frac{w_{f}}{h_{c}}
$$

or

$$
\frac{\partial \log \left[C\left(h_{c}\right)\right]}{\partial t}=\frac{w_{f}}{h_{c}}
$$

Discretizing the time derivative and rearranging gives the following linear equation for the settling velocity:

$$
\log \left[C\left(h_{c}\right)_{t=t_{1}} / C\left(h_{c}\right)_{t=0}\right]=\frac{w_{f}}{h_{c}} \Delta t
$$

where $C\left(h_{c}\right)_{t=0}$ is the initial concentration after the waves have passed and $\Delta t$ is the time difference between when the concentration was measures at $t=0$ and the concentration at arbitrary time $t=t_{1}$. In this analysis, $C\left(h_{c}\right)_{t=0}$ 
will be the maximum concentration after a wave event. The data show that the concentration then decays through time, and a linear regression of Equation (24) produces a slope that is equal to $w_{f} / h_{c}$. The fall velocity is then estimated by multiplying the slope by the depth of the sensor. Equation (24) assumes a linear vertical concentration gradient and a constant settling velocity during the time period used to generate the best fit line. Regression analysis produces confidence limits to gauge the accuracy of these assumptions. An error estimate for the slope, $b_{s}=w_{f} / h_{c}$ is given by

$$
b_{s}\left(1-e_{s}\right)<b_{s}<b_{s}\left(1+e_{s}\right)
$$

where:

$$
e_{s}=t s_{\alpha / 2, n-1}\left[\frac{1}{n-2}\left(\frac{1-r^{2}}{r^{2}}\right)\right]^{1 / 2}
$$

and $t s$ is the Student's t-distribution for the $(1-\alpha)$ confidence interval, $n$ is the number of observations, and $r$ is the regression coefficient.

The settling velocity equation is for individual size classes; however, it is common practice to combine a range of particle size classes and define the settling velocity for that group (Manning and Dyer 1999). The LISST measurements are sorted according to size class, and the four size classes with the highest concentrations are averaged to define $C$. The geometric mean is applied because the spacing between size classes is logarithmic. The particle size representative of the four highest concentrations is determined as the weighted mean:

$$
d_{m}=\frac{\sum_{i=1}^{4} C_{i} d_{i}}{\sum_{i=1}^{4} C_{i}}
$$

where:

$$
\begin{aligned}
C_{i} & =\text { concentration in size class } i \\
d_{i} & =\text { particle diameter in size class } i .
\end{aligned}
$$




\subsection{Vessel wake measurements}

Boat wake was generated with a 21-feet (ft)-long Boston Whaler Outrage. A vessel passage event during the field study is illustrated in Figure 3.3. In all experiments, the boat sailing line was parallel to the channel axis but at different distances from the bank. The exact distance and vessel speed were not recorded as the main objective of the study was to investigate sediment transport processes and hydrodynamics due to vessel waves. A total of 38 vessel passes were conducted over the 2-day study. Because of the burst sampling protocol ( $15 \mathrm{~min}$ on $/ 5 \mathrm{~min}$ off), the individual vessel passages were synchronized with the ADVs. The maximum number of vessel passages during a single burst was five and the minimum was one. The last nine vessel passages occurred during high tide, so the water depth over the sensors was too deep to produce a measureable wake signature, leaving a total of 29 vessel wake events. The passes occurred at different tidal stages and different ambient flow velocities in order that the hydrodynamics and sediment dynamics associated with vessel waves could be investigated in terms of variable tidal forcing.

Figure 3.3. Vessel wake impinging on the saltmarsh at mid-tide when the bank is submerged. The white marker denotes the location of the instrument frame. The ADVs are approximately $0.5 \mathrm{~m}$ below the water surface.

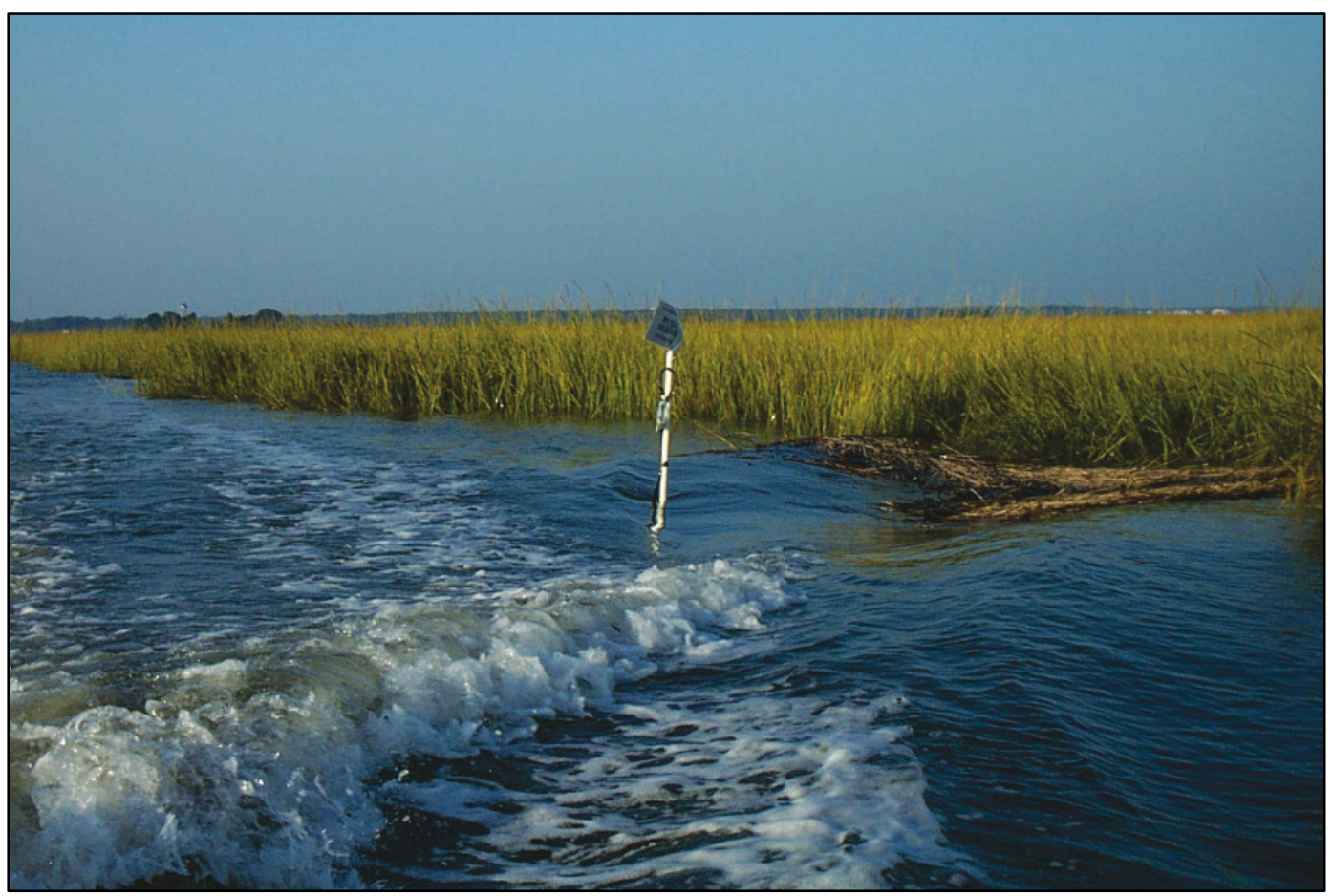




\subsection{Sediment concentration}

Water samples were collected during the course of the experiment and analyzed for total suspended sediment (TSS). Samples were acquired using 1-liter Nalgene bottles by placing the capped bottles adjacent to the ADV sampling volumes and then removing the caps and allowing them to fill underwater. Eighteen samples were collected adjacent to each probe, for a total of 36 samples. The lower ADV (N246) was sampled first, and then the upper ADV (N230) was sampled approximately 30 s later. The time to fill the bottle was approximately $10 \mathrm{~s}$. The precise time (to $1 \mathrm{~s}$ accuracy) that the cap was removed was recorded and later correlated with the ADV clock for regression analysis. To capture a range of concentrations, samples were acquired at different stages of the tide and under different vessel wake conditions.

The samples were returned to the laboratory for TSS analysis. Each sample was filtered through a 1.5-micron glass filter. The filter was wet weighted and then dried at $100{ }^{\circ} \mathrm{C}$ in a gravity-convection oven for $24 \mathrm{hr}$. TSS was calculated as the difference between the dry filter weight before and after the analysis.

\subsection{Sediment analysis}

Acoustic backscatter measurements can be converted to TSS using regression analysis techniques. The relationship between acoustic backscatter and suspended sediment concentration can be written as a regression equation:

$$
\log _{10}(C)=m A B S+b
$$

where:

$$
\begin{aligned}
C & =\text { measured concentration } \\
A B S & =\text { acoustic backscatter intensity from ADV } \\
m & =\text { calibration constant } \\
b & =\text { calibration constant }
\end{aligned}
$$

The samples were regressed against the acoustic backscatter data from each ADV to determine the fitting parameters $[m, b]$. The results of the regression indicate a positive correlation between concentration and acoustic backscatter for both instruments, with N246 producing a higher 
overall regression coefficient $(r=0.70)$ compared to N230 $(r=0.33)$ (Figures 3.4-3.5). The corresponding p-values are 0.001 and 0.18 for N246 and N230, respectively. The lower correlation coefficient combined with the higher $\mathrm{p}$-value for N230 indicates less confidence in the calibration for this instrument. Therefore, the regression equation is not used to model concentration for N230. The regression equation is applied to N246 to produce a modeled time series of the suspended sediment concentration that is synchronized with the velocity measurements.

Figure 3.4. Calibration of acoustic backscatter and concentration measurements for instrument N246. The instrument was $0.22 \mathrm{~m}$ above the bottom.

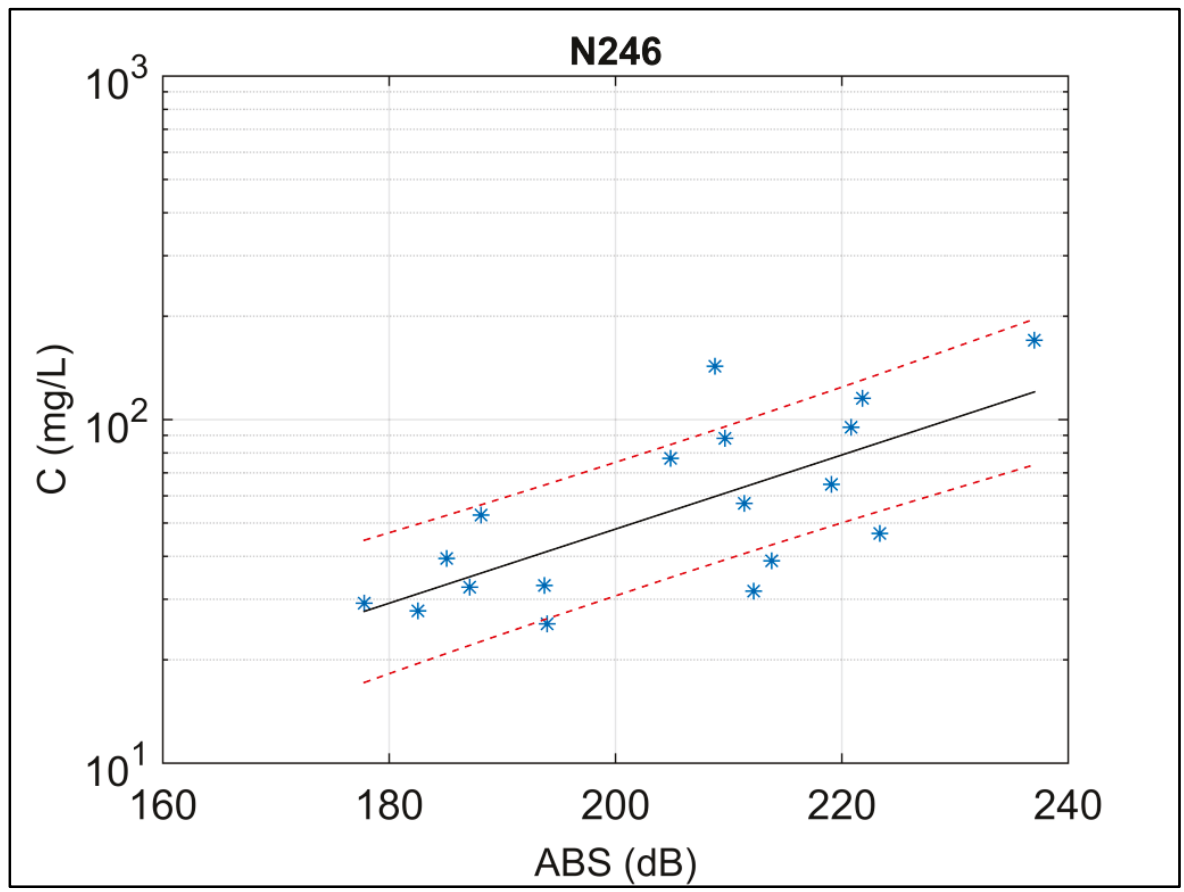


Figure 3.5. Calibration of acoustic backscatter and concentration measurements for instrument N230. The instrument was $0.40 \mathrm{~m}$ above the bottom.

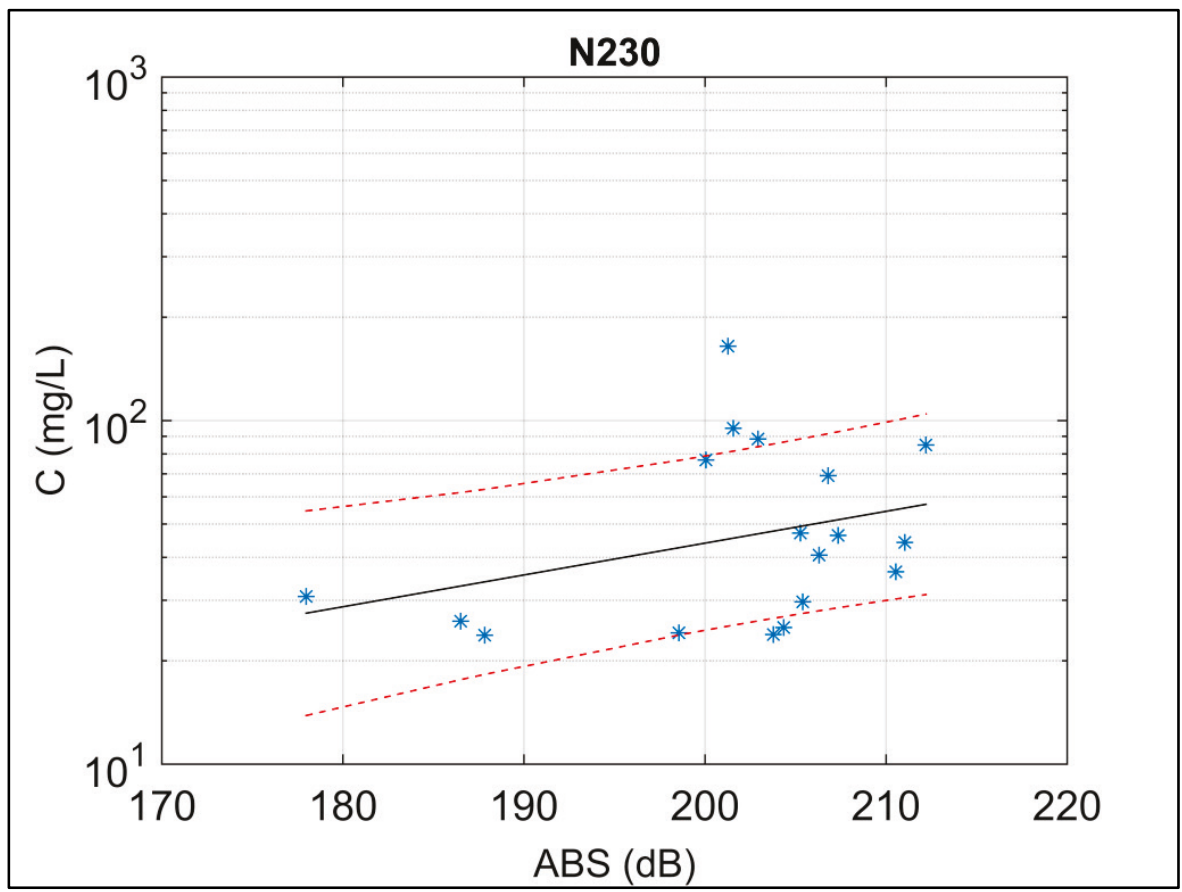

\subsection{Reynolds stress, turbulence kinetic energy (TKE) production, and dissipation estimates}

The Reynolds stress $(\tau)$ is written in terms of the kinematic shear stress $u_{*}^{2}=\tau / \rho$. The kinematic shear stress, which is the square of the shear velocity $\left(u^{*}\right)$, is computed using the direct covariance method of the fluctuating velocity components:

$$
u_{*}^{2}=-\left\langle u^{\prime} w^{\prime}\right\rangle-\left\langle v^{\prime} w^{\prime}\right\rangle
$$

where the brackets represent a temporal average over a burst. In shear-generated boundary layer flows, the TKE production $(P)$ is written as

$$
P=-\left\langle u^{\prime} w^{\prime}\right\rangle \frac{\partial U}{\partial z}-\left\langle v^{\prime} w^{\prime}\right\rangle \frac{\partial V}{\partial z}
$$

Estimates of shear production are computed using the vertically stacked ADVs by substituting the derivatives in Equation (30) with the horizontal velocity difference between the two sensors and dividing by the vertical separation distance. 
In shear-generated boundary layer flows TKE dissipation approximately balances production, allowing dissipation to be written as

$$
\varepsilon=\frac{u_{*}^{3}}{\kappa z}
$$

where:

$$
\begin{aligned}
& \varepsilon=\text { TKE dissipation } \\
& \kappa=\text { von Karman's constant }(=0.4)
\end{aligned}
$$

\subsection{Shear parameter and Kolmogorov micro-scale}

Suspended particles in fine-grained sediment environments tend to form cohesive flocs (Winterwerp and Van Kesteren 2004). Neglecting particle collisions, floc size is limited by the magnitude of the turbulence shear stresses that tend to break up flocs. Two factors used in the study of cohesive sediment dynamics are the dissipation parameter $(G)$,

$$
G=\sqrt{\frac{\varepsilon}{v}}
$$

and the Kolmogorov micro-scale $\left(\lambda_{0}\right)$,

$$
\lambda_{0}=\left(\frac{v^{3}}{\varepsilon}\right)^{1 / 4}
$$

The Kolmogorov micro-scale is a measure of the size of the smallest turbulent eddies and therefore the maximum sustainable floc size. Flocs of greater diameter are subject to turbulence stresses that can overcome the cohesive bonding properties of individual grains, thereby limiting floc growth. At scales less than $\lambda_{0}$, viscous effects dominate, and the sediment cohesive properties can more easily maintain floc stability. Typical values obtained in estuaries range from 100 to 1000 microns (Winterwerp 1998). The dissipation parameter represents the velocity rate of strain and is proportional to the local shear. Higher rates of strain produce greater shearing force causing the breakup of flocs. Laboratory experiments conducted by Manning and Dyer (1999) show reductions in average floc size from 150 to 80 microns as $G$ increases from 12 to $45 \mathrm{~s}^{-1}$. 


\subsection{Wavelet analysis}

A wavelet transform can be used to analyze time-series data that contain nonstationary variability that occurs over multiple timescales (Daubechies 1990). A wavelet analysis is akin to time-series spectral analysis such as the well-established Fourier transform method (Press et al. 1989). Unlike spectral analysis, which applies the basis functions to the full time record, the time domain is divided into discrete and usually overlapping segments. The spectral analysis is applied independently to each segment. In this way, it is possible to analyze a signal whose spectral characteristics evolve through time.

Wavelet analysis has a strong appeal for elucidating the spectral signature of time series containing turbulence fluctuations that are intermittently interrupted by vessel passage. Traveling vessels produce a narrower-banded wave packet with finite temporal duration in an otherwise wider-band turbulence signal. Furthermore, wavelets can also detect vessel wake in the presence of wind generated waves. Even if the frequency characteristics of the wind-generated waves are similar to the boat wake, the wind waves will vary on a much longer time scale compared to the duration of the boat wake. Thus, the finite time window of the wavelet analysis is ideal for extracting the spectral characteristics of the vessel wake even in the presence of wind waves and turbulence. Once the spectral signature of the vessel wake is identified, then the spectrum can be integrated to give the wave height variance, and the location of the spectral peak can be inverted to give the wave period.

Wavelet analysis is performed using the "Cross wavelet and wavelet coherence toolbox for MATLAB” (Grinsted et al. 2004). The wavelet analysis program is written in Matlab, and the user must have the basic Matlab software to run the wavelet program. The package produces the energy density as a function of time and period. The analysis is applied to each burst and used to isolate the wave period and wave energy of the individual vessel wakes. 


\section{Hydrodynamics and Sediment Concentration Measurements}

This chapter describes the processes associated with vessel wake sediment resuspension in a fine-grained sediment environment. Initially, the tidal hydrodynamics are presented to place the vessel wake measurements in the proper context considering that tidal flow also influences sediment dynamics in this environment. This is followed by a presentation of the vessel wake results.

\subsection{Flow measurements}

The burst average water surface elevation ( $\eta$ ), along channel current, and suspended sediment concentration are depicted in Figure 4.1. The first data gap in the record denotes low tide, in which the water level drops below the height of the sensors. The second gap denotes the recovery from the oyster bank and redeployment over the mud bank. These gaps persist through all of the time-series data presented and any calculated parameters that arise from the data. Water surface elevation shows that the study covered three and a half tidal cycles. The maximum surface elevation indicates a mixed tidal regime with a high-high tide followed by a low-high tide. The current magnitude peaks during the rising or falling limb of the tide. Current speed is higher at $40 \mathrm{~cm}$ above the bed indicating a sheared region typical of a tidal boundary layer. The highest currents are on the second day and are skewed towards flood tide. Minimum concentration is approximately 20 milligrams per liter $(\mathrm{mg} / \mathrm{L})$ and occurs near slack tide. The concentration increases with flow speed, with the highest concentrations during flood tide on day two of the study. 
Figure 4.1. Flow measurements. The height above the bed (hab) denotes the distance from the bed to the ADV sampling volumes $(\mathrm{N} 246=22 \mathrm{~cm}, \mathrm{~N} 230=40 \mathrm{~cm})$. Positive values denote flood currents and concentration time series is derived from N246.

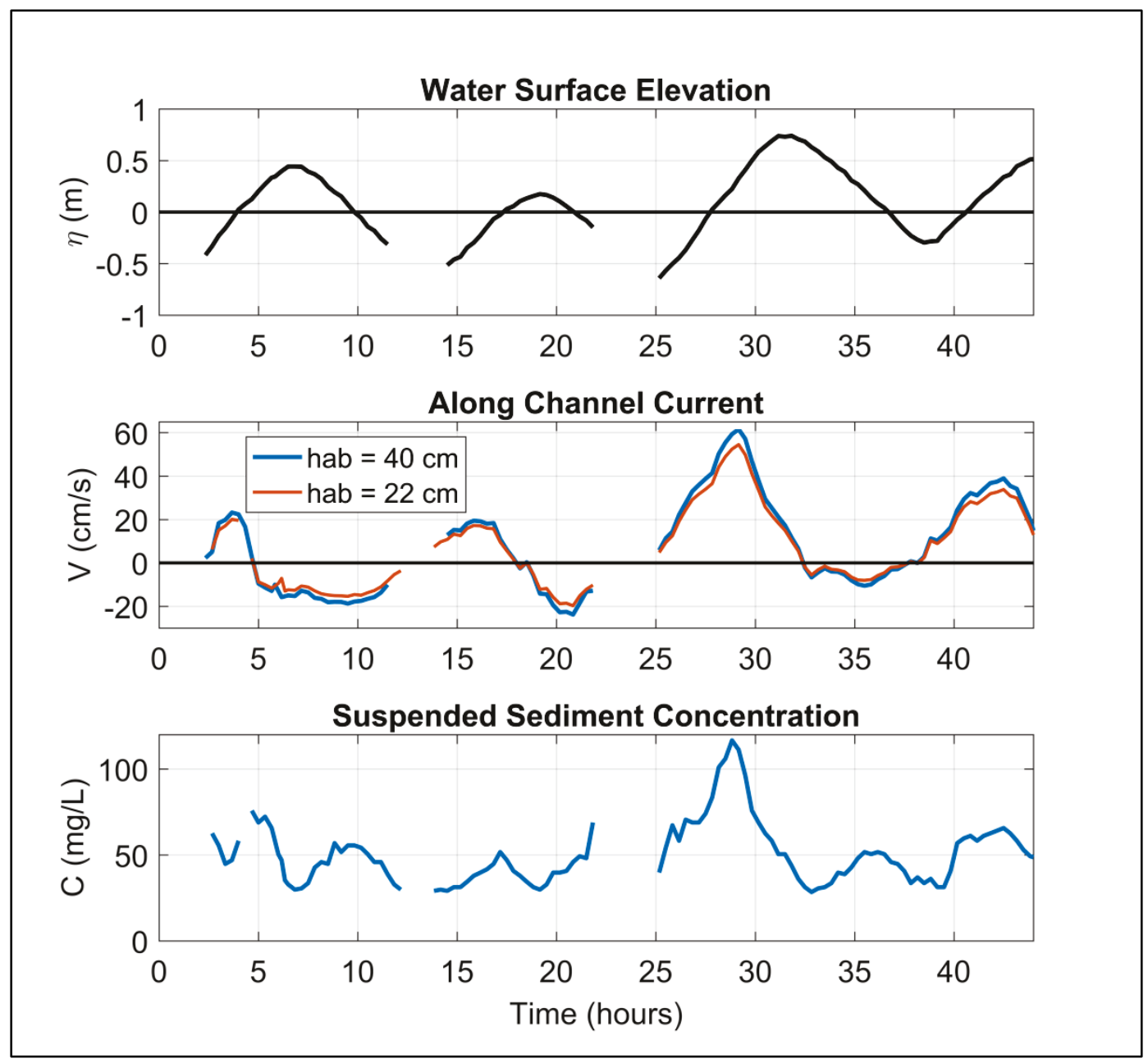

\subsection{Turbulence quantities}

The kinematic shear stress is generally lower during the first two tidal cycles (Figure 4.2). The largest kinematic shear stress occurs during the third tidal cycle, which also has the largest currents. During maximum flows the kinematic shear stress is higher at the lower sensor height. This is consistent with greater shear-generated turbulence near the bed. TKE production and dissipation are similar with the exception of maximum flows during the last two tidal cycles, in which production exceeds dissipation. 
Figure 4.2. Time series of turbulence parameters. The kinematic shear stress increases closer to the bed and increases with flow strength.

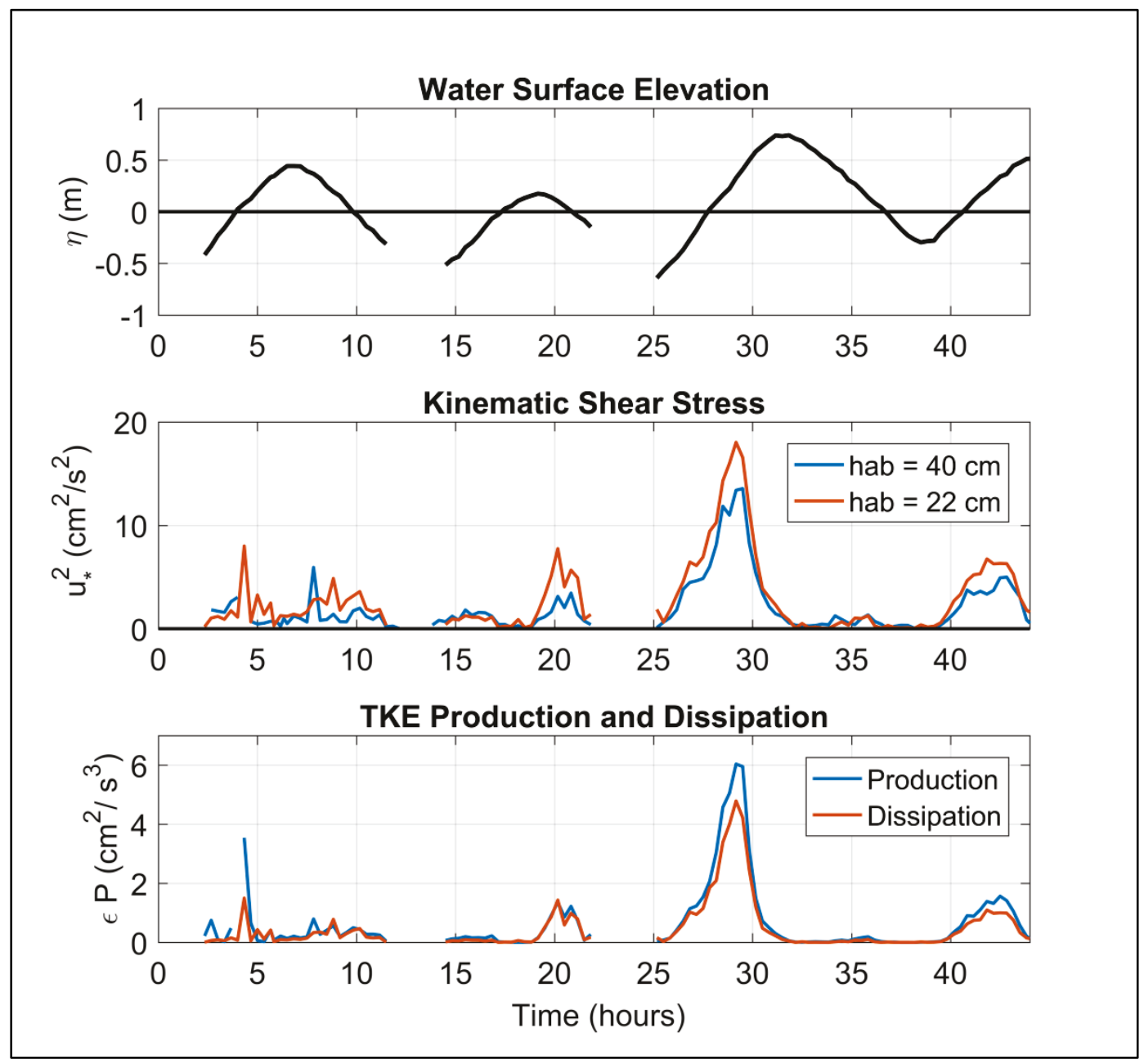

\subsection{Sediment parameters}

The Kolmogorov micro-scale tends to be larger than the particle size associated with the LISST (Figure 4.3). The Kolmogorov micro-scale denotes the largest floc size whereas the LISST is a measure of the particle size that represents the highest concentration. Furthermore, the LISST is restricted to a maximum size of 500 microns due to the sampling range of the instrument, so any larger flocs that may be present are not detected. During the first day, the LISST shows on average larger particles during the second tidal cycle. During the second day, the smallest particles occur during the rising limb of the flood tide when currents are strong and $G$ is largest. Average particle size increases near slack high tide as $G$ decreases due to a reduction in vertical shear as the along channel current diminishes. Average particle size decreases during the falling limb of the ebb tide as current 
increases. The shear parameter increases during this time indicative of greater vertical shear associated with the current and the developing tidal boundary layer.

Figure 4.3. Time series depicting sediment parameters. Particle size denotes the theoretical maximum floc size based on the Kolmogorov micro-scale and the LISST particle size associated with the highest concentration. Shear parameter is a measure of the level of turbulence needed to break up flocs.
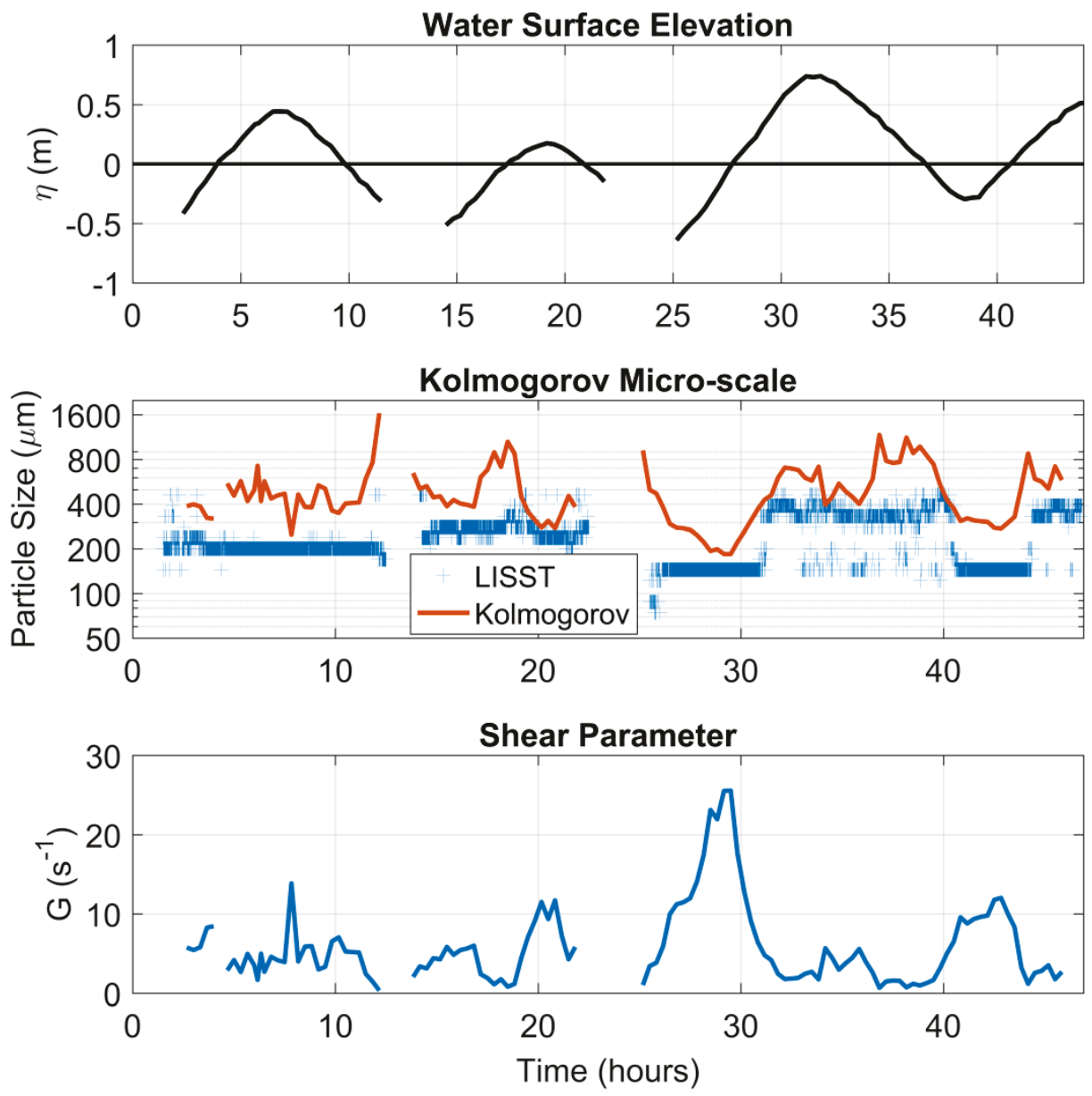

Regression analysis of the burst-averaged concentration and kinematic shear stress shows a positive correlation as measured by the $p$-value (Figure 4.4). The results imply a dynamic equilibrium in which increases in bed shear stress are able to maintain higher concentrations in the water column. Thus, the tidal flow plays a primary role in controlling the sediment dynamics of the saltmarsh and should be considered when investigating bank erosion. 
Figure 4.4. Regression analysis of suspended sediment concentration as a function of the kinematic shear stress. The variables represent the average conditions for each burst and thus reflect the effect of the tidal currents and not vessel wake. Solid line denotes best fit.

Dashed lines denote an estimate of the standard deviation of the error in predicting a future observation.

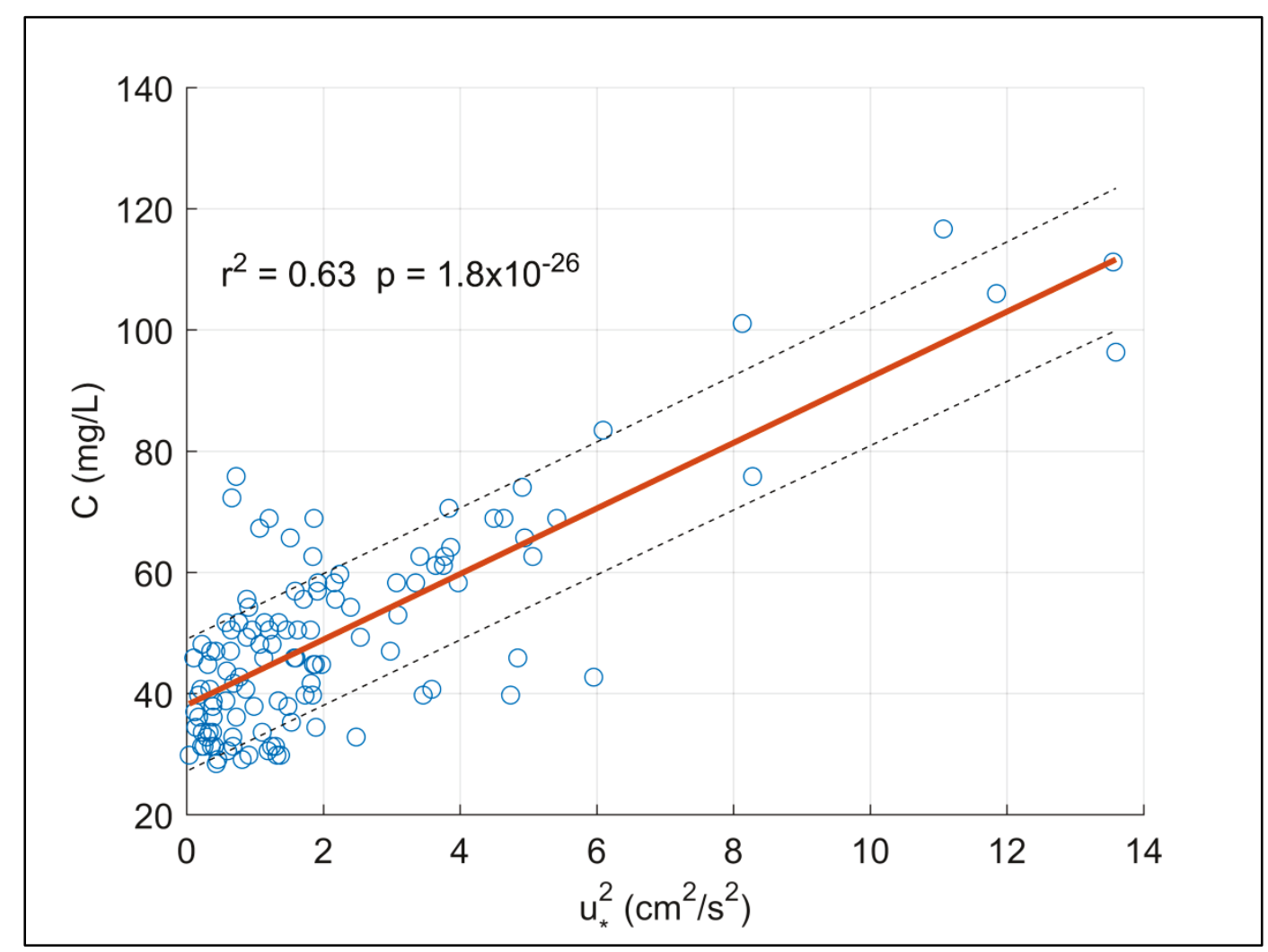

\subsection{Wake measurements}

An example illustrating two vessel wake events is depicted in Figure 4.5. Vessel-induced wake is identified in the cross-shore current time series at approximately $3 \mathrm{~min}$ and $7 \mathrm{~min}$, respectively, into the record. The first passage generates the largest velocity amplitude. Concentration before the first wake event averages approximately $28 \mathrm{mg} / \mathrm{L}$. It then increases after the first vessel passage in a series of large fluctuations until approximately $5 \mathrm{~min}$, after which time it begins to decrease until the second wake event. A second increase at approximately 8 min occurs just after the second wake event. The concentration then decreases for the remainder of the burst. The increase in concentration is not smooth and contains large amplitude fluctuations superimposed and an overall initial increase followed by a relatively steady decrease. The negative slope of the concentration versus time following the second wake event is a measure of the settling rate of the suspended material. In this case, the concentration has not returned to background levels by the end of the burst. The delay in 
maximum concentration to maximum wave velocity is likely due to wave breaking on the shoreline backing the instruments. This would produce a time delay between the breaking wave and the sensor reading, due to advection of the turbid water from the shore to the measurement point. Figures depicting the velocity and concentration for all bursts with vessel wake are presented in Appendix A.

Figure 4.5. Time series of cross-shore velocity and sediment concentration for a 15-min burst that included two vessel passage events (denoted by blue arrows). The vertical axis is in the correct units for concentration and speed. The increase in velocity fluctuations clearly identify vessel wake. Concentrations increase after each vessel passage indicating wake-induced sediment resuspension.

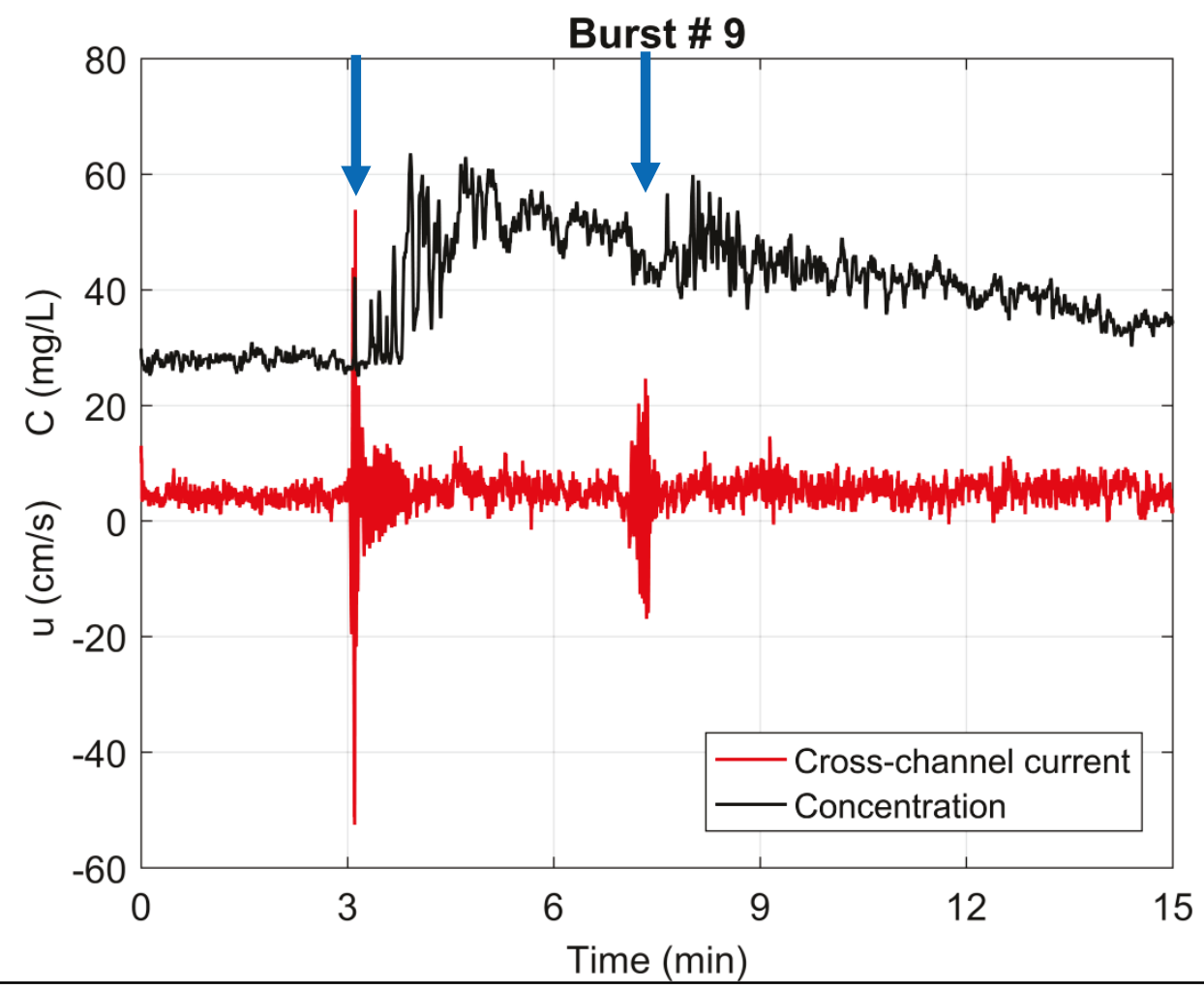

For reference, Appendix B contains a table listing some of the computed wake height parameters including burst number, average water depth, wave period, bottom orbital velocity, and wave height. This information contributes to the body of existing wake height parameters and is made available to test or validate wake models in future studies. This is a crucial factor as the results presented in this study likely represent the first dataset for vessels operating within the interior regions of coastal plain saltmarshes. 


\subsubsection{Wavelet analysis}

The wavelet analysis provides a way to visualize the change in the spectral characteristics of the velocity time series, which helps delineate the wave period and amplitude of individual wake events. As a reminder, wavelet analysis decomposes a time signal into its frequency components and then, using a moving window, produces a time-series representation of a spectral signal. The $u$-component of velocity is chosen for analysis because waves generated by a vessel traveling along the axis of the channel produce larger velocity fluctuations in the cross-channel coordinate compared to the along-channel coordinate. The energy density $\left(E_{u}\right)$, which represents the cross-shore kinetic energy in each period (frequency) band, shows isolated peaks corresponding with the two vessel passages (Figure 4.6). The time series has been high-pass filtered using a $20 \mathrm{~s}$ window to dampen low-frequency fluctuations. This makes it easier to identify vessel-induced velocity fluctuations and removes large eddies and infra-gravity motions, which should be decoupled from the wake-induced motion to make it easier to identify. Filtering also removes low-frequency motion that is outside the cone of influence due to truncation of the time series. For instance, a signal with a frequency of $4 \mathrm{~min}$ cannot be resolved during the first or last 2 min of the burst record because the wavelet uses a symmetric time-centered weighting function to compute the spectra. This is not an issue for vessel wake, with typical periods of less than 10 s so long as the vessel passage does not occur during the first or last $5 \mathrm{~s}$ of the record, which is never the case in this study. Appendix $\mathrm{C}$ includes figures of the wavelet results for all bursts with vessel wake.

Focusing on the second wake event ( 7 min into the record) reveals the fine-scale structure of the wake-induced flow and helps to illustrate the advantage of wavelets for analyzing signals with energetic spectral signatures that vary with time (Figure 4.7). The boundaries of the wake event are defined by the $20 \mathrm{~cm}^{2} / \mathrm{s}^{3}$ energy density contour, indicating that the event lasts from 7.1 to $7.4 \mathrm{~min}$-or it takes approximately $18 \mathrm{~s}$ for the wave train to pass over the sensor. At $7.1 \mathrm{~min}$ into the record, the wave period associated with the maximum energy density is just under $4 \mathrm{~s}$, but by the end of the wake passage $(7.4 \mathrm{~min})$, it is reduced to approximately $2 \mathrm{~s}$. Also during this time, the energy density increases, reaching a maximum between 7.3 and $7.4 \mathrm{~min}$. The asymmetry in the long axis of the energy density contours indicates that the amplitude decreases more rapidly on the trailing side of the peak $(7.35-7.4 \mathrm{~min})$ than the rising side of the peak $(7.1-7.35 \mathrm{~min})$. 
The original time series aids in interpreting the wavelet analysis as it clearly illustrates the decrease in the spacing between zero-crossings and the increase in amplitude between 7.1 and $7.35 \mathrm{~min}$. However, the wavelet analysis makes it easier to assign a wave period to the representative vessel wake and more easily illustrates the shift in peak period as the wave train passes. It also better resolves the period associated with the highest amplitude waves, which is important in sediment transport studies.

Figure 4.6. Wavelet analysis for a burst that included two vessel passages. Time series of high-pass filtered cross-shore velocity helps to identify vessel passage events.

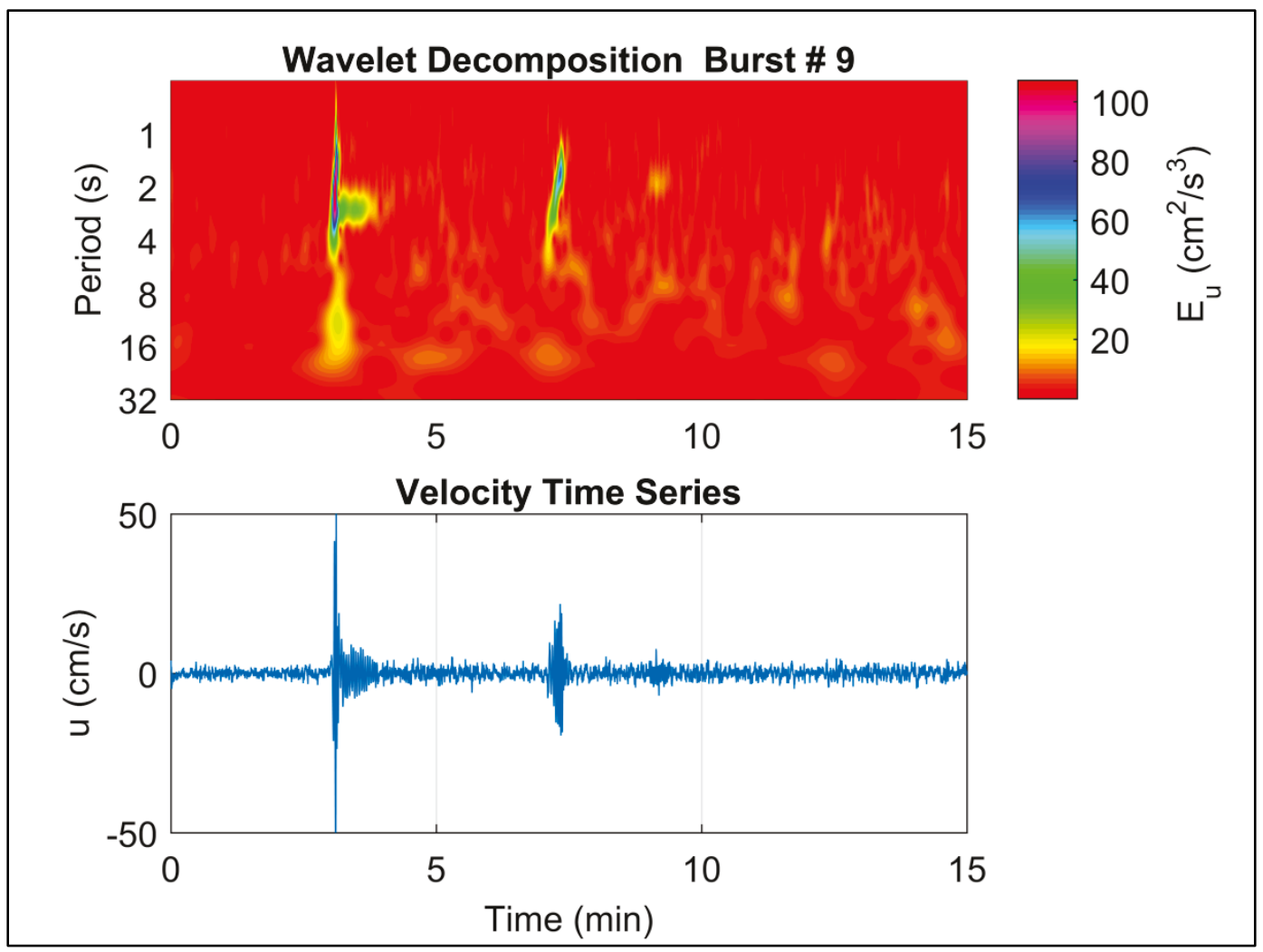


Figure 4.7. Wavelet analysis illustrating a single-vessel passage event. The upper panel depicts the spectral decomposition of the velocity time series. A major feature is the shift from longer to shorter periods as the wave train passes. The lower panel is the original cross-shore velocity time series.

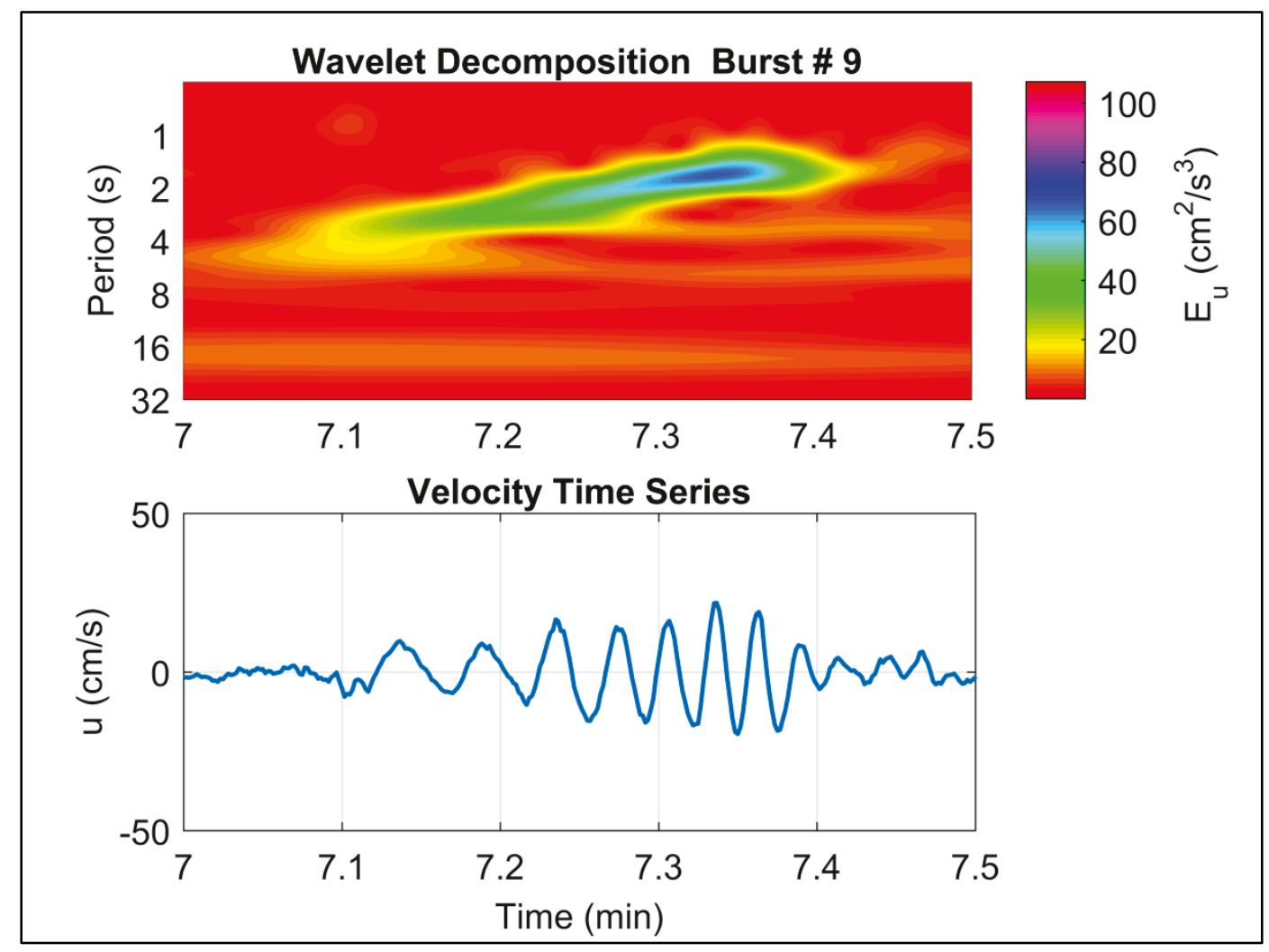

\subsubsection{Vessel-induced resuspension}

For each burst with vessel wake, the concentration is low-pass filtered, and then the maximum concentration following a wave event is extracted to define the representative wake-induced sediment resuspension. The maximum concentration establishes an upper bound on the erosion potential of vessel wake.

A regression analysis of the concentration versus the maximum shear stress under the wave shows a positive relationship $\left(\mathrm{p}=5.8 \times 10^{-6}\right)$. While positively correlated, the level of scatter in the data leads to a moderate correlation coefficient of $r^{2}=0.57$ (Figure 4.8). For modeling purposes, the error associated with the slope of the best fit line provides a measure of the predicted suspended sediment concentration distribution for a given maximum wave-generated shear stress appropriate for this setting. This information is used to establish error statistics for a vessel wake model demonstration for recreational vessels operating in North Inlet. 
Figure 4.8. Regression analysis of sediment resuspension due to maximum shear stress created by vessel wake. Solid line denotes best fit based on the linear regression model. Dashed lines denote an estimate of the standard deviation of the error in predicting a future observation.

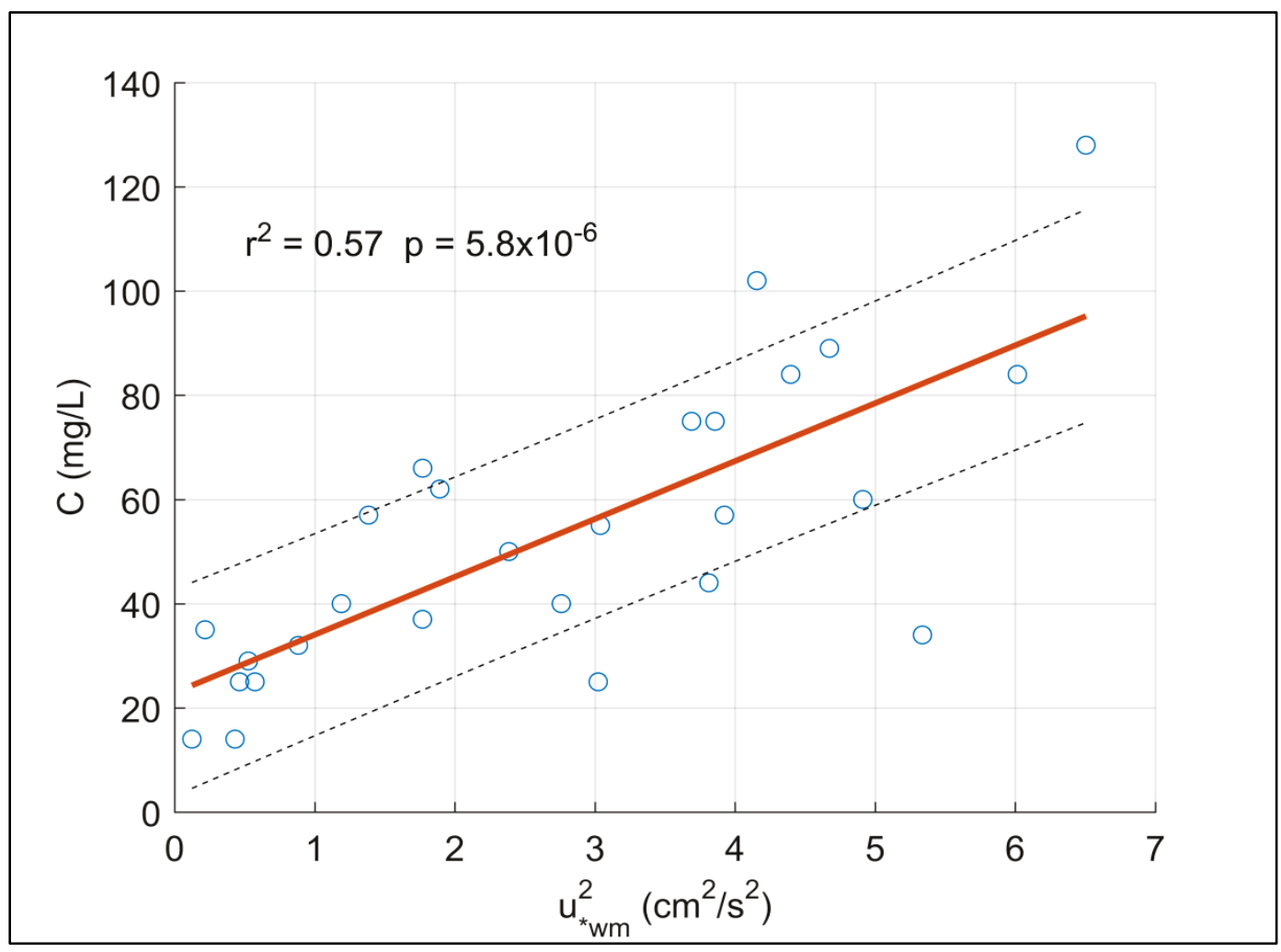

\subsubsection{Particle settling velocity}

Particle size distribution is calculated from the LISST. However, near-bed concentrations during some of the wake events are so high that the laser beam is fully attenuated and no measurement is possible. Under these conditions, the laser transmission reduces to zero and the instrument is unable to measure concentration or particle size (examples can be seen in figures provided in Appendix A). Size distribution is determined by regression analysis as described in Section 3.4. Predicted settling velocities from the three empirical models (discussed in section 2.5) bracket the majority of values estimated from the LISST (Figure 4.9). However, the differences in model predictions and in the computed settling velocities show large variability and emphasize the difficulty of predicting fall velocity from floc size. Estimates from the ADV backscatter produced settling velocities ranging between 0.2 and 2.0 millimeters $/ \mathrm{s}(\mathrm{mm} / \mathrm{s})$, which is within the range of the LISST measurements. However, concentrations derived from backscatter do not include an estimate of size, so they are not included in the plot. Even though there is variability, the results tend to be on the same order of magnitude as the models. 
Figure 4.9. Settling velocity derived from the LISST and empirical models.

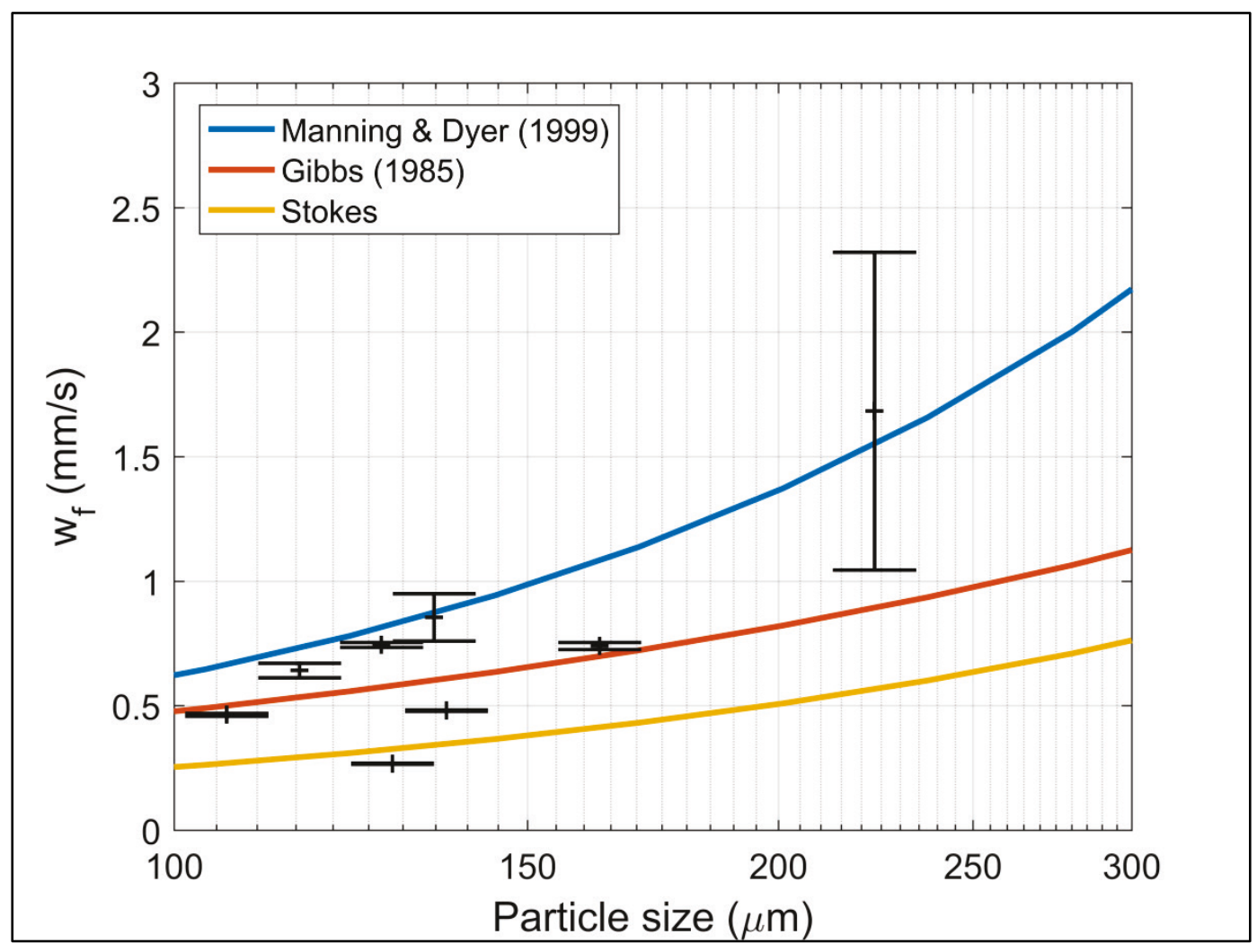




\section{Vessel Wake Model Demonstration}

The results are used to demonstrate an application of the vessel wake theory for recreational vessels operating in a coastal plain saltmarsh similar to North Inlet. The goal is to determine the relative contribution of vessel wake to shoreline erosion in comparison to the ambient tidal forcing. In many applications, vessel wake studies arise from a need to understand the likelihood of accelerated shoreline erosion and water quality degradation due to changes in navigation patterns-namely, increases in recreational or commercial vessel activity. Will increases in vessel activity accelerate shoreline erosion or promote poorer water quality, and if so, to what degree will these changes have in comparison to pre-activity conditions? A general approach (Maynord 2008) is to determine the number of vessel passages per time in a given setting and compare the cumulative energy flux to the ambient forcing in the system, in this case the tidal flow. If the energy flux due to vessels exceeds the tidal contribution, then it is possible that vessel traffic may pose additional environmental risks and further investigation is warranted. If the tidal energy greatly exceeds the cumulative effect of vessels, then the wave energy contribution from vessels is less likely to adversely affect the system. Using a distribution of hypothetical vessel traffic patterns for North Inlet, this analysis will determine the number of vessels over a tidal cycle that produce similar energy as the tidal flow as well as produce estimates of suspended sediment concentrations, bottom stress, and other parameters that may aid decision makers in assessing the environmental impacts of navigation as a component in an overall saltmarsh restoration plan or related efforts.

\subsection{Vessel type}

The demonstration focuses on a saltmarsh with similar characteristics as North Inlet. The vessel specifications are for a Boston Whaler Outrage, and the parameters needed to define coefficients in the Maynord (2005) wake height model are listed in Table 5.1. To emulate multiple vessel passages, a random number generator is used to define a range of vessel speeds. The mean and standard deviation are set by the user, and the random number generator produces a Gaussian probability distribution function based on those settings. The average speed is set to $10 \mathrm{~m} / \mathrm{s}$, which produces a wave height near the bank that is equal to the average of the calculated wave height from the field experiment. The standard deviation is set to $5 \mathrm{~m} / \mathrm{s}$ to 
give a broad speed and wave height distribution. The total number of vessel passages is treated as an independent variable as one of the tasks is to determine the cumulative energy flux and compare the result to the tide.

Table 5.1. Vessel characteristics for $21 \mathrm{ft}$ Boston Whaler Outrage used as input to the Maynord (2005) wake model.

\begin{tabular}{|l|c|c|c|c|c|c|c|}
\hline $\begin{array}{l}\text { Vessel } \\
\text { Model }\end{array}$ & Mass & Draft & Length & Beam & $\begin{array}{c}\text { Depth } \\
\text { @Vessel }\end{array}$ & $\begin{array}{c}\text { Distance } \\
\text { to Bank }\end{array}$ & $\boldsymbol{W} / 3$ \\
\hline & $(\mathrm{kg})$ & $(\mathrm{m})$ & $(\mathrm{m})$ & $(\mathrm{m})$ & $(\mathrm{m})$ & $(\mathrm{m})$ & $(\mathrm{m})$ \\
\hline $\begin{array}{l}\text { Boston } \\
\text { Whaler } \\
\text { Outrage }\end{array}$ & 1818 & 0.23 & 6.5 & 2.2 & 4.0 & 100 & 1.22 \\
\hline
\end{tabular}

A time series of water level is generated for an M2 tide with a sinusoidal variation. The tidal range is set to $1 \mathrm{~m}$, which corresponds to average M2 tidal conditions in the interior of North Inlet. The simulation time is set to a single tidal cycle in which an arbitrary number of vessel passages occur. The mean depth near the bank is $1 \mathrm{~m}$, and the mean depth in the middle of the channel is $4 \mathrm{~m}$, which correspond to conditions at the field data collection site.

An example of the vessel speed distribution as a function of depth for a total of 80 vessel passages during a single tidal cycle is depicted in Figure 5.1. Vessel speeds $<3 \mathrm{~m} / \mathrm{s}$ are discarded from the analysis, which is the lower limit used to calibrate the Maynord (2005) model. This plot represents a possible scenario depicting vessel activity during a tidal cycle typical of a shallow saltmarsh sub-tidal creek. The number of vessel passages is variable and can be set to reflect traffic patterns in a particular saltmarsh setting. Alternatively, the data could reflect the results of a field program in which vessel activity is monitored over a tidal cycle, or as the case here, the number of vessels and speeds are chosen stochastically to represent a reasonable range of conditions. 
Figure 5.1. Hypothetical vessel speed distribution over a tidal cycle. The tidal range is $1 \mathrm{~m}$ with a mean of $4 \mathrm{~m}$ depth at the location of the vessel. The plot represents 80 vessel passages at different phases of the tide to emulate possible conditions for a coastal plain saltmarsh similar to North Inlet.

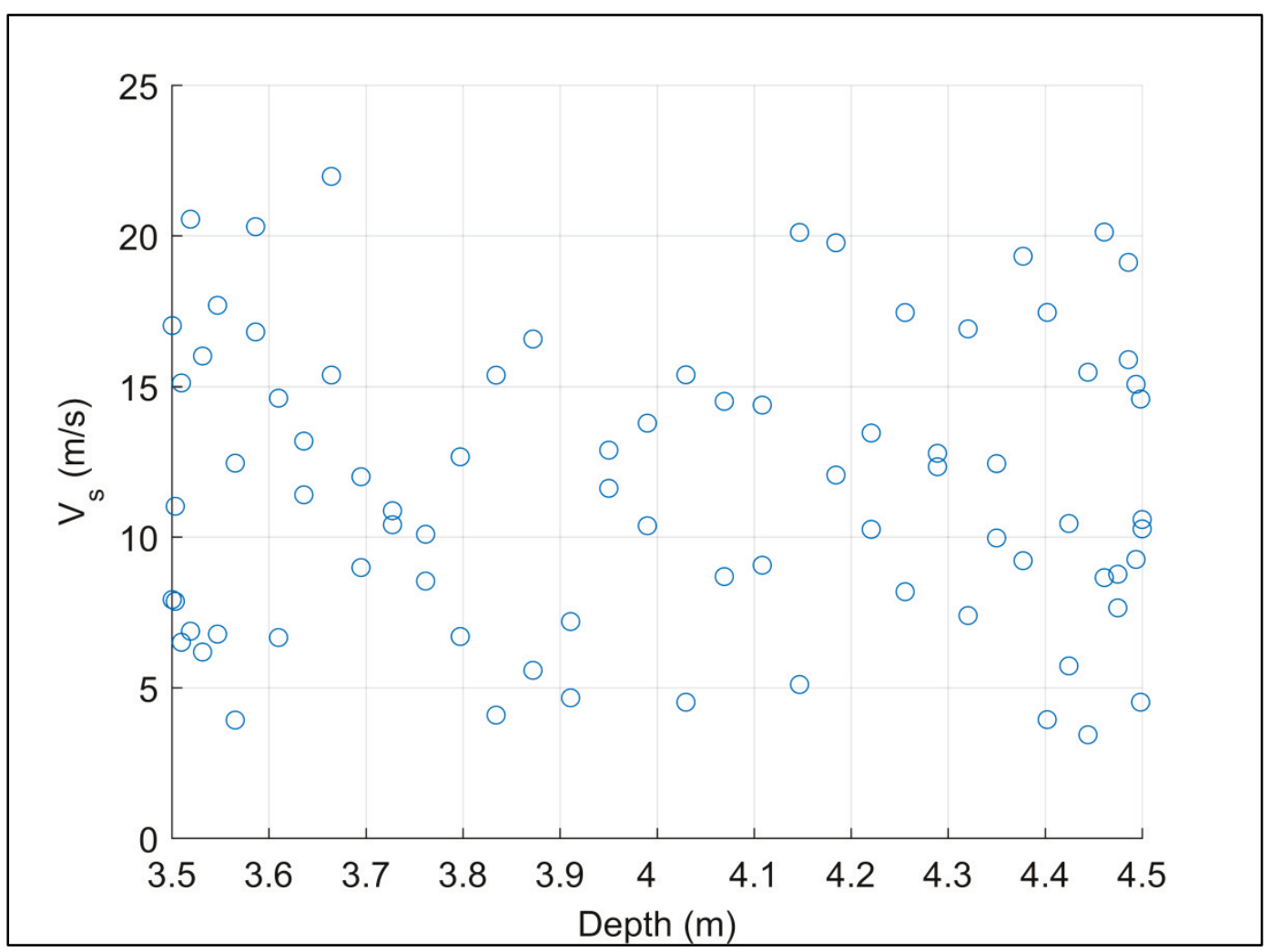

\subsection{Wake and sediment predictions}

Using the above values for vessel speed and the specifications for the Boston Whaler listed in Table 5.1, the Maynord (2005) model wave height predictions at the bank are depicted in Figure 5.2. The distribution indicates a peak in the 0.09 to $0.11 \mathrm{~m}$ band. The range of calculated wave heights from the field test is listed in Table B.1 in Appendix B is 0.03 to $0.24 \mathrm{~m}$, which brackets the predicted values.

The mean water depth and tidal range near the bank are set to $1 \mathrm{~m}$ and $0.5 \mathrm{~m}$, respectively. This ensures the minimal water depth $(0.5 \mathrm{~m})$ does not violate the linear wave assumption used to compute the wavelength, and associated bottom orbital velocity and shear stress, yet is shallow enough to represent conditions near the shoreline. The range of calculated kinematic shear stress is depicted in Figure 5.2. The first two bins account for $80 \%$ of the total vessel passage events, with median kinematic shear velocities of 2.5 and $7.6 \mathrm{~cm}^{2} / \mathrm{s}^{2}$, respectively. The computed range of shear velocities from the measurements is 0.1 to $6.5 \mathrm{~cm}^{2} / \mathrm{s}^{2}$, which correspond to these first two bins that account for the majority of vessel passage events. 
Figure 5.2. Wave height and kinematic shear stress distribution over a tidal cycle.
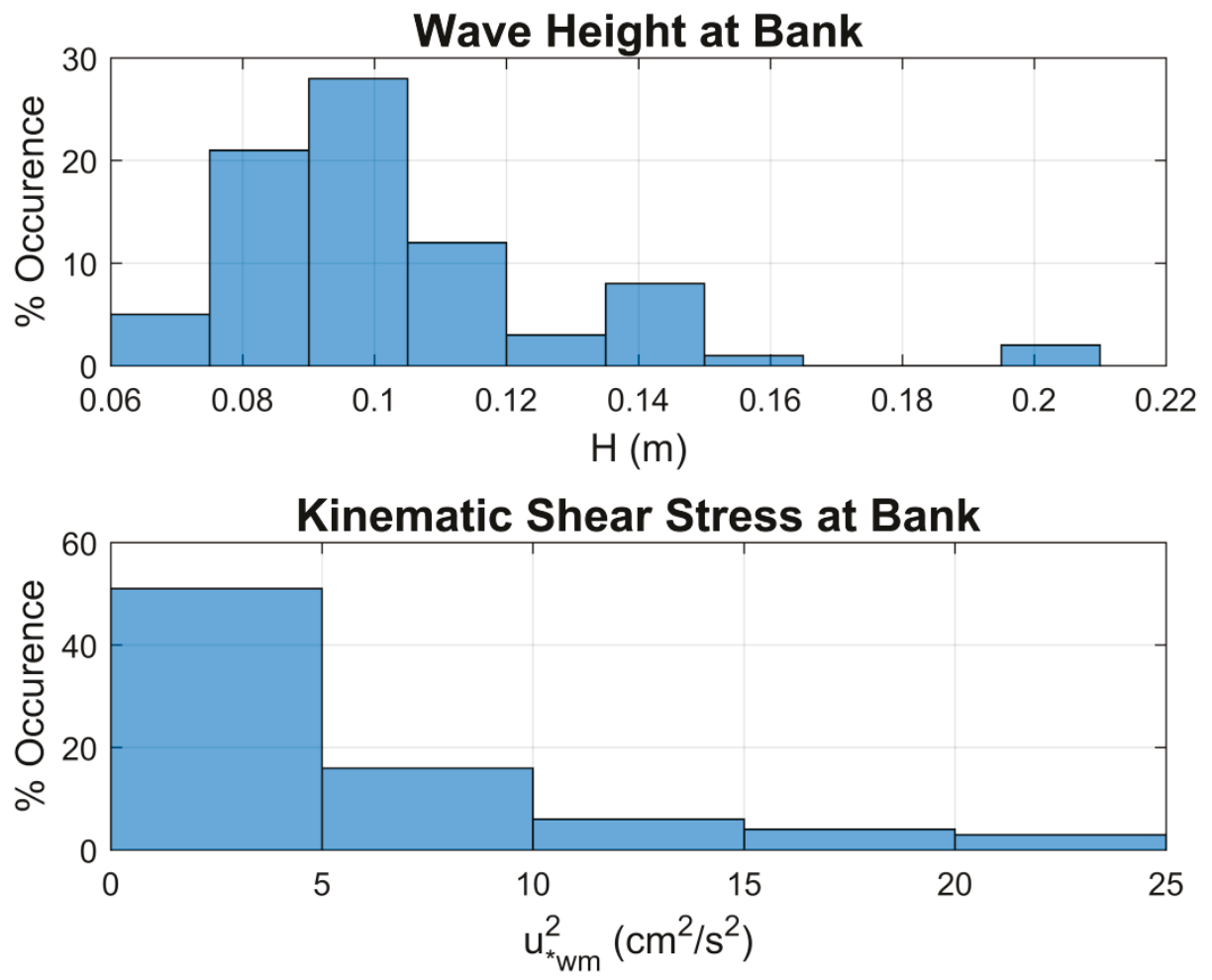

\subsection{Suspended sediment concentration}

Suspended sediment concentration is predicted using a regression model derived from the best-fit curve of concentration versus maximum kinematic shear stress depicted in Figure 4.8. The modeled concentration is applicable to North Inlet as the regression coefficients are generated from data obtained locally and therefore are specific to this field setting. Bed sediment properties, grain size, density, and sediment composition can vary widely leading to large variability in sediment concentration predictions. When possible, sediment models should be validated or calibrated locally to minimize uncertainty.

Because the model is calibrated using acoustic backscatter measurements derived from the lower ADV (N246), the results represent the maximum wave-generated concentration at $22 \mathrm{~cm}$ above the bed. The total suspended sediment load would need to be computed using a onedimensional model to represent the vertical distribution of sediment concentration. This is a practical consideration, but for illustrative purposes, the analysis focuses only on the concentration at the specified height. It is noted that sediment concentration is higher near the bed so 
that the model is representative of near-bed conditions. The distribution indicates that over $60 \%$ of the near-bed concentration is less than 100 $\mathrm{mg} / \mathrm{L}$ (Figure 5.3). This is well within the range of tidally induced sediment resuspension as measured during the field program (Figure 4.4). Maximum concentration due to tidal currents is on the order of $100 \mathrm{mg} / \mathrm{L}$, but the average is $50 \mathrm{mg} / \mathrm{L}$ (Figure 5.4). Maximum concentration from vessels is $450 \mathrm{mg} / \mathrm{L}$, but it occurs less than $3.0 \%$ of the time. With the exception of a few instances in which concentrations are greater than the maximum associated with the tide, resuspension due to vessels is the same order of magnitude to what is generated by the tidal current.

Figure 5.3. Suspended sediment concentration predictions. The results represent the suspended sediment concentration measured at $0.22 \mathrm{~cm}$ above the bed.

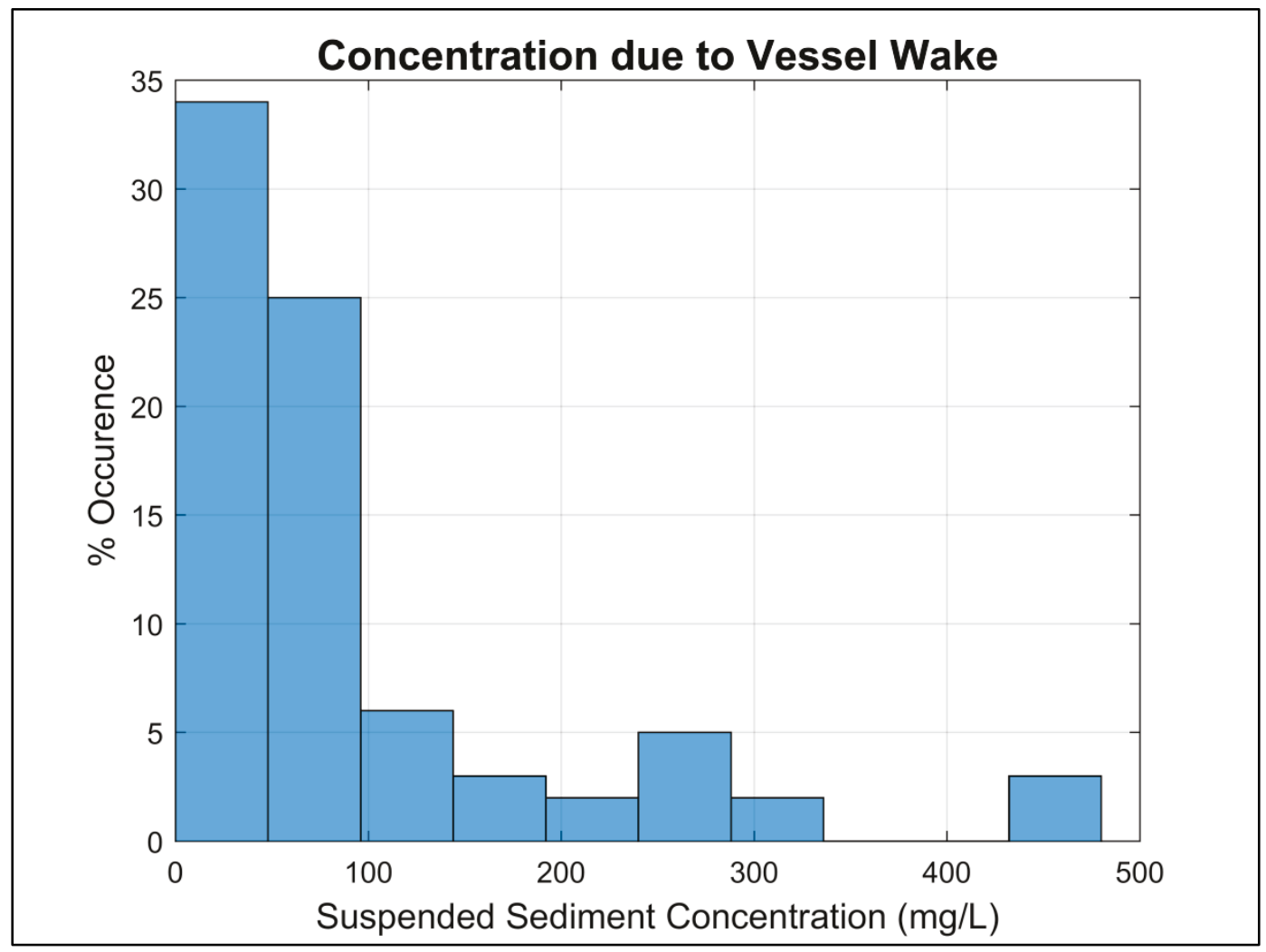


Figure 5.4. Distribution of concentration due to tidal flow computed from the ADV acoustic backscatter.

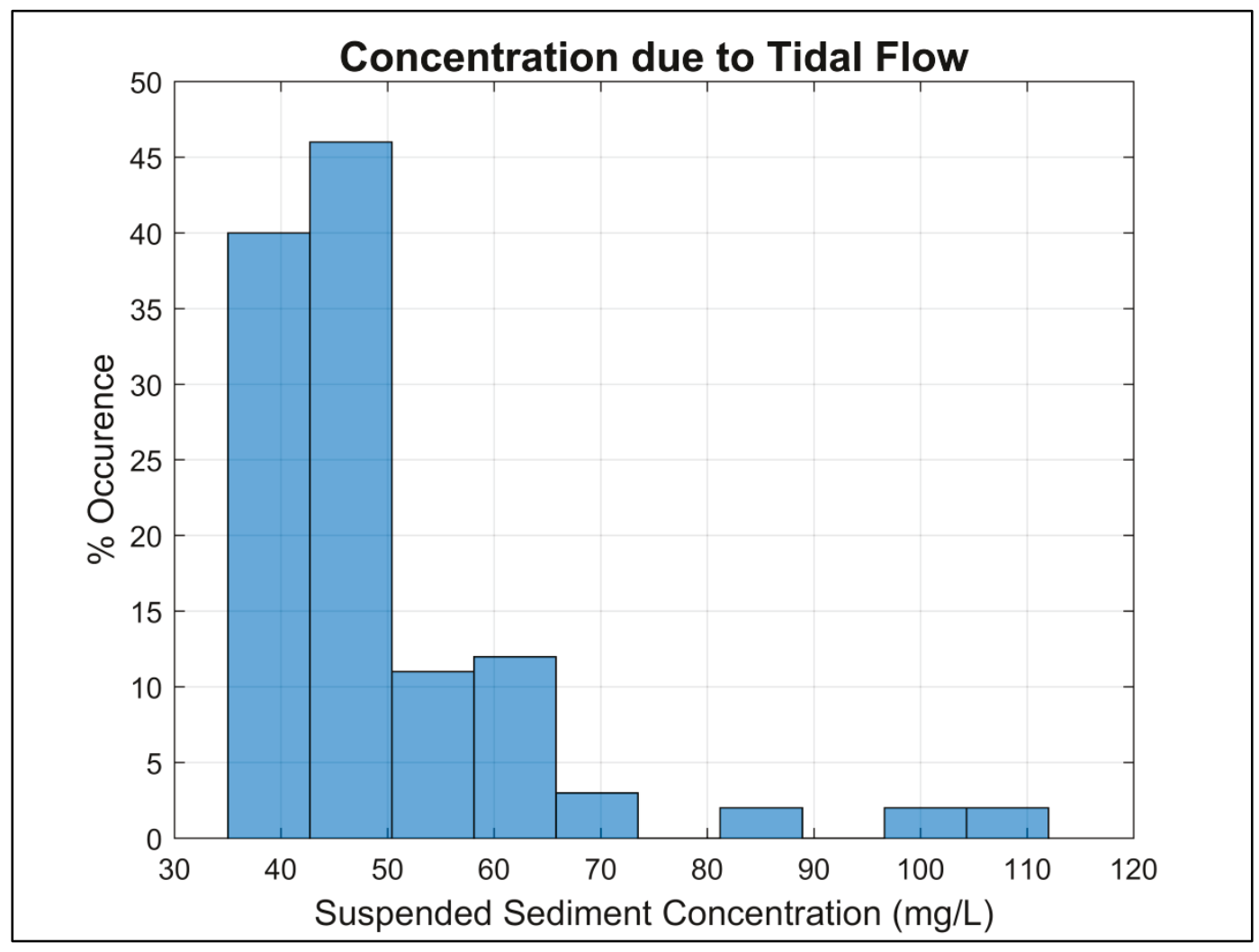

\subsection{Energy flux}

The total energy per unit area for a tide with amplitude $A$ can be written as

$$
E_{t}=\frac{1}{2} \rho g A^{2}
$$

which is the same expression as the energy in any linear wave. However, only a fraction of the total tidal energy is dissipated due to bottom friction. The energy loss per unit time due to bottom friction is equal to the bottom stress multiplied by the tidal current:

$$
E_{t d}=\rho c_{d} U_{0}^{3} \sin ^{3} \sigma t
$$

where:

$$
\begin{aligned}
U_{\mathrm{o}} & =\text { tidal velocity amplitude } \\
c_{d} & =\text { non-dimensional drag coefficient } \\
\sigma & =\text { tidal radian frequency }
\end{aligned}
$$


A quadratic drag law is used to define the bottom stress, and the sine function represents the oscillatory tide (Miller 1966). Integrating the absolute value of Equation (35) over a tidal cycle gives the average energy dissipation due to bottom stress:

$$
P_{t}=(4 / 3 \pi) \rho c_{d} U_{0}^{3}
$$

This is the energy dissipated over a tidal cycle per unit planform area $\left(\mathrm{W} / \mathrm{m}^{2}\right)$. Of course, the total tidal energy distribution across the saltmarsh and integrated over a tidal cycle greatly exceeds the vessel wake energy. However, the local energy loss due to bottom friction per unit area per unit time is very small compared to the total tidal energy.

To compute the dissipation per unit length of channel, which is comparable to the energy loss due to the vessel wake, Equation (36) should be multiplied by the average channel width $\left(b_{c}\right)$,

$$
P_{t}=(4 / 3 \pi) b_{c} \rho c_{d} U_{0}^{3}
$$

However, this would overestimate the energy dissipation responsible for bank erosion as it includes energy dissipated in the subtidal portion of the channel away from the bank. Previous work in rivers (Hill et al. 2002; Maynord et al. 2008) suggests only a small fraction of the cross-channel energy flux $(1 \%-5 \%)$ contributes to bank erosion. Noting that the energy dissipation due to vessel wake is for one bank only, the portion of the channel width contribution to bank erosion should be equal to $b_{c} / 2 \times$ (0.01-0.05).

To compare the tidal dissipation to the vessel wake, Equation (37) is multiplied by the tidal period $(=12.42$ hours $\times 3600 \mathrm{~s} /$ hour $)$ to give the total energy dissipated per unit width over one tidal cycle. Based on data from this report and previous studies (Torres and Styles 2007; Voulgaris and Meyers 2004), representative values for the terms in Equation (37) for North Inlet are $U_{0}=0.5 \mathrm{~m} / \mathrm{s}, c_{d}=0.003, b_{c}=100 \mathrm{~m}$. Adopting these values produces a tidal energy loss of 13.1 kilojoules $/ \mathrm{m}$ per tidal cycle.

For vessel wake, the energy flux for a single wave is determined using Equation (5). The total flux is the sum of all waves that occur during a given timeframe multiplied by their individual period, 


$$
E_{w}=\sum_{1}^{N} E_{f i} T_{i}
$$

where $N$ is the number of waves. In this case, it is the total number of waves generated during the tidal cycle. Vessel-generated waves travel in discrete wave packets, so it is necessary to add up the energy due to each individual wave. A direct calculation is impractical as the model predicts the energy of the highest wave whereas the wave packet contains a number of waves of different amplitudes. Furthermore, the number of waves in a wave packet increases with distance from the sailing line, but the amplitude decays (Maynord 2005) making it difficult to accurately represent the energy flux. A procedure is developed to account for the combined amplitudes and numbers of waves in a wave packet using a single representative wave.

The number of waves in the wave train is difficult to predict, so an estimate is determined based on observations of the field data. The average number of waves per vessel-wake event is eight, with a standard deviation of 3.5. The model predicts the maximum wave height, which is not representative of the entire wave packet as all other waves have smaller amplitudes. Simply multiplying the predicted wave height by the number of waves overestimates the energy flux. To compensate for the overall lower wave energy, the number of waves could be artificially reduced and then multiplied by the computed energy flux for a single wave to estimate the total. However, the maximum value leads to an upper bound on the potential energy flux due to vessel wake and is the approach used in this demonstration.

Estimating the number of waves using the above argument is debatable as it lacks strong experimental support. The stochastic nature of the exercise allows the number of waves to be treated as a variable. This increases the uncertainty in the predictions but provides a consistent approach that can be refined in the future as new information becomes available and a better model of the number of waves in a wave packet can be developed. Additionally, this simplified approach is designed as a screening or planning activity to investigate the potential effects of vessel wake on the environment. As such, some degree of error is inherent in the model approach presented in this report. If the error analysis indicates unacceptable levels of uncertainty for a given application, as gauged by model sensitivity to relatively small changes in the magnitude of empirical 
coefficients and other constraints, then more rigorous methodologies can be employed that reduce the number of unknowns and provide a more robust statistical approximation. The individual situation will dictate the level of acceptable risk, which is determined by the needs of the project.

As an example calculation, the energy flux is computed following the procedures described above but with the number of vessels operating in the saltmarsh increased in increments of 10 from 10 to 100 vessels per tidal cycle. For each vessel group, the model is run 100 times to produce a distribution of energy flux. Equation (38) is used to sum the total number of waves $(N=10,20,30, \ldots, 100)$, and the result is multiplied by the number of waves per group. The average energy flux assuming eight waves per group increases nearly linearly as a function of the number of vessels (Figure 5.5). The results indicate that once the number of vessels surpasses $\sim 55$ per tidal cycle, the total energy flux due to vessels exceeds the energy flux associated with the tide. Over a $12.42 \mathrm{hr}$ tidal cycle, this translates to almost five vessels per hour. This is a very high number compared to the first author's observations of one or two vessels per day in North Inlet during previous field studies. On holiday weekends, traffic density could possibly exceed five vessels per hour, but it would likely be confined to daylight hours and would not be sustained indefinitely whereas the marsh always experiences two tides per day. A monitoring effort could quantify vessel activity in North Inlet or other areas and provide a definitive estimate of vessel passage statistics. A similar analysis as presented here could be compared to the results of a monitoring effort to determine the likelihood of recreational vessel impacts in comparison to the ambient tidal flow. 
Figure 5.5. Energy dissipated over a tidal cycle assuming eight waves per wave packet. The horizontal dashed line is the tidally averaged energy dissipation. Error bars denote \pm one standard deviation.

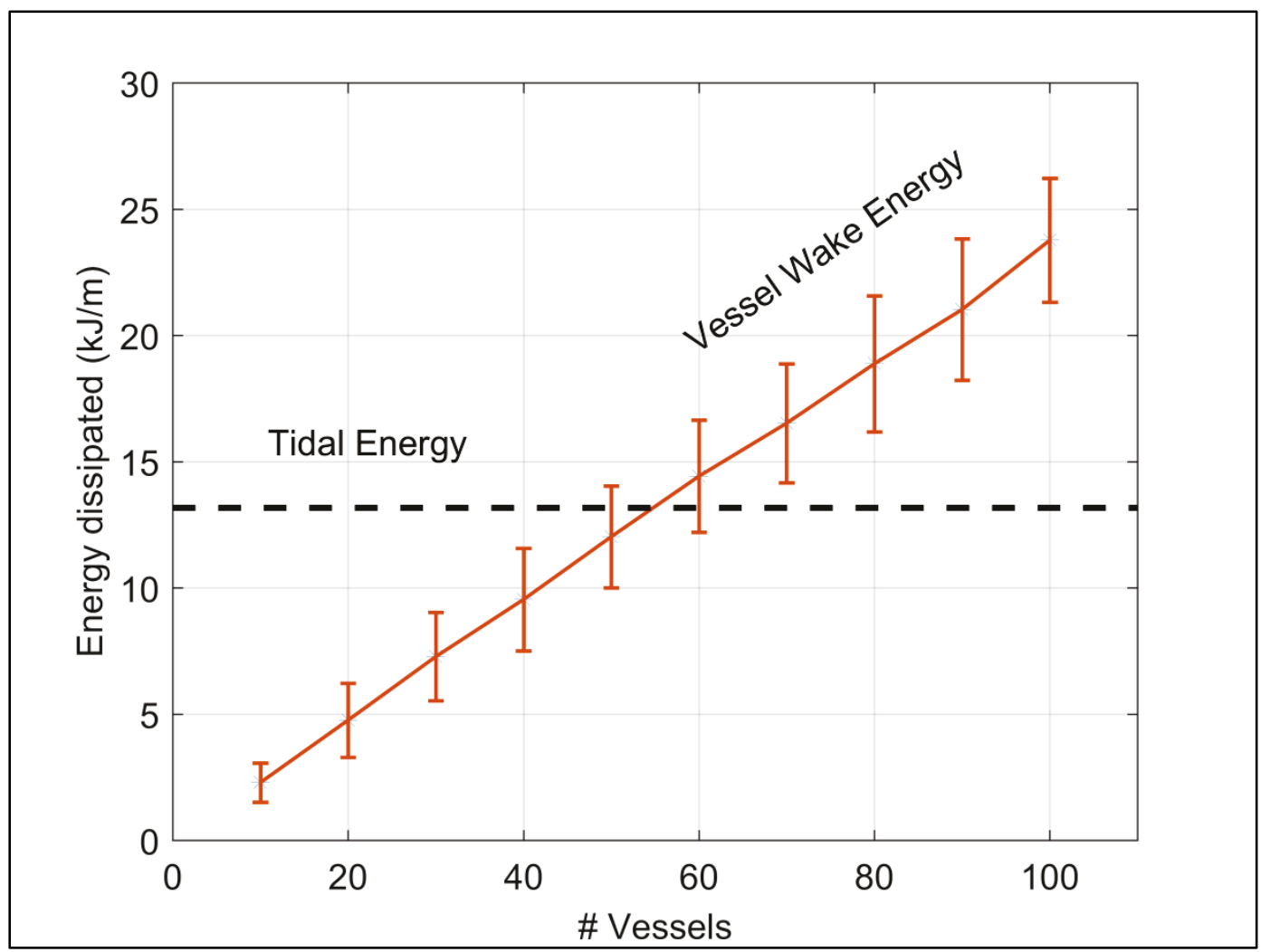

The greatest unknown is the number of waves in a wave packet. Increasing the number of waves increases the wave energy flux linearly. If a greater number of waves per packet were used in the analysis instead of eight, then a lower number of vessels would produce an equivalent energy flux as the tide. The results for 10 waves per packet shows that the wave energy flux intersects the tidal energy flux at approximately 45 vessels per tidal cycle, or 3.6 per hour (Figure 5.6). This is still a relatively high number not likely sustainable under current recreational usage of North Inlet.

However, it does provide a quantitative estimate to inform environmental managers responsible for assessing the potential effects of increases in recreational vessel activity on the saltmarsh. 
Figure 5.6. Energy dissipation over a tidal cycle for 10 waves per wave group. The horizontal dashed line is the tidally averaged energy dissipation.

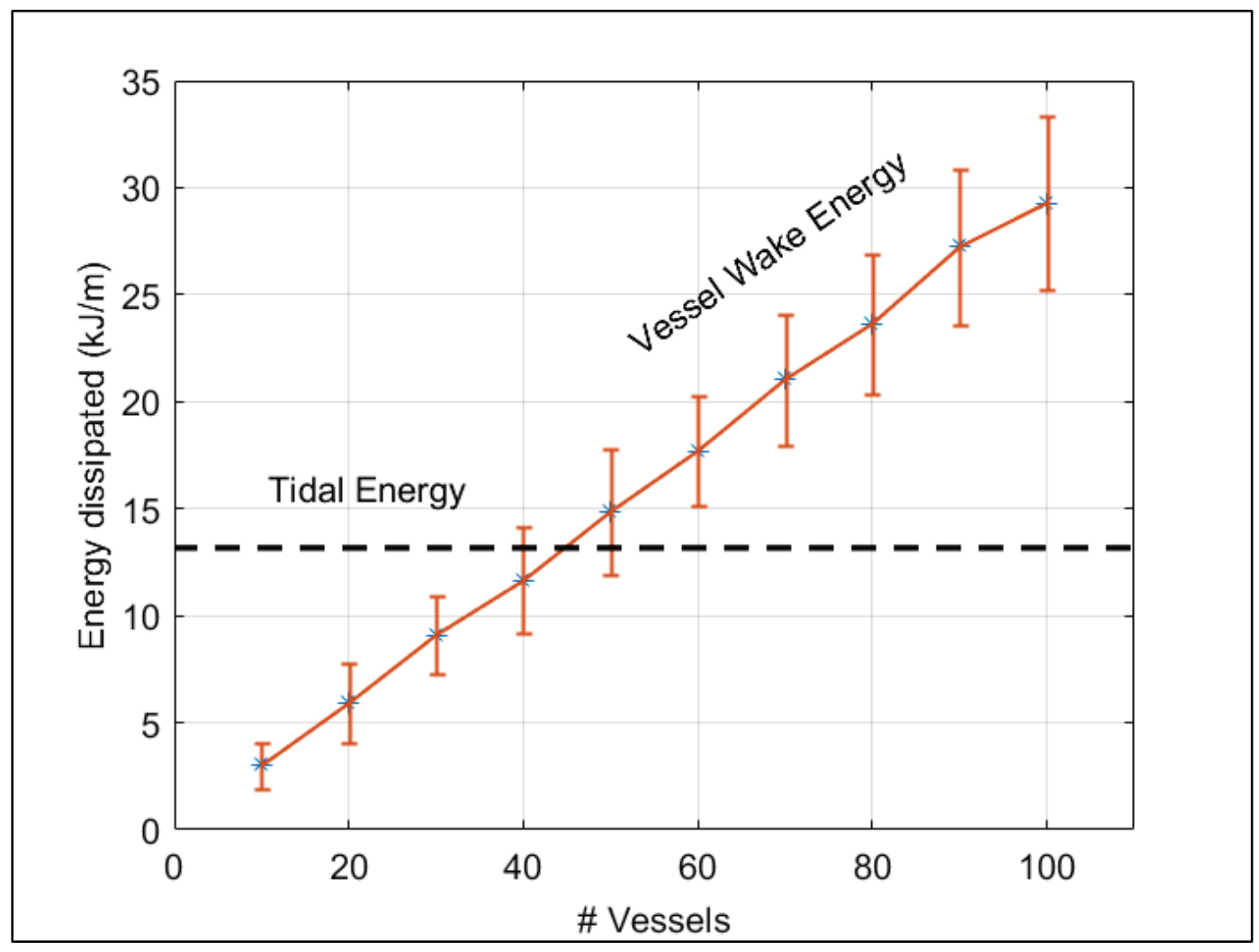




\section{Discussion}

General comments regarding some of the salient features discovered in the analysis are presented to better understand processes that could provide important information regarding vessel-wake effects in tidal saltmarshes and other regions where simple vessel-wake models can be applied. These issues are also important in understanding the degree to which vessel wake can adversely impact a natural environmental system, which could lead to overestimation of the relative importance of vessel impacts by not considering other factors such as tidal phase and ambient forcing.

\subsection{Water level in an intertidal marsh}

Water level has a first-order influence on the degree of sediment resuspension and bank erosion. The data show that when the water level is below the height of the marsh platform, the vessel wake impinges on the bank and breaks. This mechanism releases the wave energy along the sloping bank, producing high concentrations of resuspended sediment in the nearshore. The higher the initial waves the greater the concentration of resuspended material. The greater the bank exposure the more likely that vessel wake, via run up and breaking, can rework the bed, leading to a greater chance for erosion. Conversely, if the water depth exceeds the marsh platform height, then the waves propagate over the bank and into the marsh interior. Even though the wave orbital velocities may still generate stresses on the submerged bank, the degree of energy loss is greatly reduced compared to breaking waves. The majority of the wave energy propagates over the marsh platform, where it is attenuated by bottom friction and increased drag caused by the marsh vegetation (Anderson et al. 2011). Additionally, deeper water over the bank reduces bottom orbital velocities and associated bed shear stress so that for a given wave characteristic (height, period), resuspension is reduced as water depth increases.

The vessel passages during bursts 15 and 16 (Figures A.4-A.5) indicate orbital velocities measured by the ADVs, yet there is minimal or no correlated increase in near-bed concentrations. The maximum orbital velocity during burst 13 is $26.05 \mathrm{~cm} / \mathrm{s}$, and there is a clear increase in concentration after the vessel passage (Figure A.3). In comparison, the maximum orbital velocity during burst 15 is $29.07 \mathrm{~cm} / \mathrm{s}$, which would suggest suspended sediment concentrations on the order of what is 
measured during burst 13. The water depth during burst 13 is less than 1.5 $\mathrm{m}$, which is the approximate height of the marsh surface. The water depth for bursts 15 and 16 exceeds the marsh platform height indicating that the waves, despite producing measureable orbital velocities, propagate over the bank and into the marsh interior. These waves produce orbital velocities on the order of the previous burst that indicated a correlation between waves and sediment resuspension, but because of the increased water depth they do not break on the bank. As such, recreational vessels traveling through the inter-tidal saltmarsh near high tide likely produce lower suspended sediment concentrations at the shoreline and have less of an effect on bank erosion.

\subsection{Sedimentation in oscillatory flows}

Another consideration is the relative contribution of vessel-induced resuspension in comparison to the background concentration. During maximum flood or ebb, energetic tidal currents resuspend the greatest amount of material. The regression analysis shows a linear correlation between concentration and the tide-generated shear stress, which indicates a dynamic equilibrium between the upward turbulent flux and gravitational settling. The highest concentrations (120 mg/L) occur on day 2 when the flood currents are the greatest and correspond to a kinematic shear stress of $14 \mathrm{~cm}^{2} / \mathrm{s}^{2}$. The highest concentration during a wake event is $130 \mathrm{mg} / \mathrm{L}$, which is similar to maximum concentrations for the tide. However, the maximum stress is only $6.5 \mathrm{~cm}^{2} / \mathrm{s}^{2}$. The lower stress for the wave that produces similar maximum concentrations is likely due to wave breaking, which produces greater mixing as all the wave energy is rapidly dissipated at the bank compared to the lower energy loss due to bottom friction associated with a passing wave (Komen et al. 1996).

The fact that maximum concentrations under vessel wake and tidal currents are similar, despite the differences in the resuspension mechanism, alludes to a possible hydrodynamic or geological constraint that modulates sediment dynamics in oscillatory flow settings such as tide dominated saltmarshes. Cohesive beds develop as layers, with varying resistance properties as a function of depth (Gibson et al. 1967). Newly deposited material has the highest water content and less inter-particle bonding. Fresh deposits are essentially flocs that settle out when the shear stress falls below the critical value to maintain sediments in suspension. These sediments have greater porosity and lower compaction. As such, they will erode more easily. Over time, new material is deposited on top of old. The 
increased weight reduces the water content of the deeper layers and increases the internal bonding strength of individual particles. The deeper the layer, the greater the bonding strength through compaction, leading to a higher erosional shear stress. Once the surface layer is removed, these deeper layers will require a higher shear stress before they will start to erode. Studies have shown that the critical shear stress for the initiation of sediment motion for cohesive sediment can vary two orders of magnitude depending upon the level of compaction (Manning and Dyer 1999).

In low-energy environments, the degree of compaction can be much higher for surface sediments because the longer wait time between resuspension events allows the surface layer to consolidate through microreworking of the interstitial layers and gravity (Been and Sills 1981). Over time, the critical shear stress for erosion may increase. In a tidal environment, surface material is eroded each tidal cycle and then re-deposited at slack tide. The surface consists of a relatively easily erodible low-density porous layer that is reworked on a periodic basis. This produces a layered structure in which an easily erodible surficial layer overlies a more compacted layer with a higher critical erosion threshold. As such, typical shear stresses produced by the tide and the vessel wake easily erode the surficial layer but not the deeper layers.

The bursts with multiple vessel passages provide evidence that this may be an artifact of tide-dominated saltmarsh systems. The maximum shear stress for the vessel wake is lower than the maximum stress for the tide, despite the fact that both mechanisms produce approximately the same maximum concentration. In cases with multiple vessel wake passages, the first wave group generally produces the largest net increase in concentration compared to background levels. The remaining wave groups show some increase in concentration, but part of this is comprised of residual concentrations from the first wave group as the particles have not completely settled out of suspension. This implies that the remaining wave groups are vertically mixing some of the same sediment resuspended by the first wave group. The initial vessel-wave event resuspends the easily erodible surface layer, and the remaining wave groups primarily rework this layer. Despite this variability in concentration and stress, the maximum resuspension during lower water levels when the banks are exposed and therefore the highest concentrations are measured remains similar to maximum concentrations during maximum tidal currents. Therefore, the wave-induced stress is sufficient to erode the surface layer 
but cannot penetrate the deeper layers. Variability in suspended sediment concentrations has been observed in North Inlet (Traynum and Styles 2007) that correlates with the tidal forcing. This material is resuspended each tidal cycle during maximum flood and ebb, which indicates the presence of a relatively easily erodible surface layer that responds to the periodic change in bottom shear stress.

An additional constraint on the amount of material resuspended could be associated with bed type. At the first location, the shoreline is covered by oysters, which anchor the sediment and reduce flow within the interstitial layers between individual oyster columns (Whitman and Reidenbach 2012). The sheltering effect may also act to reduce sediment resuspension by impeding the penetration of the overlying turbulent flow between individual organisms and by anchoring sediments at their base. Consolidation and oysters embody a bed armoring mechanism in that they restrict the maximum sediment available for resuspension.

This raises an issue regarding vessel traffic patterns in areas vulnerable to shoreline erosion such as estuaries and wetlands and other regions with large concentrations of fine-grained material. The demonstration study shows that the maximum shear stress is between 30 and $35 \mathrm{~cm}^{2} / \mathrm{s}^{2}$. This is more than twice the maximum due to tidal currents. While these higher stresses occur during a small fraction of the tidal cycle, they could potentially erode not only the surficial layer but the deeper, more compacted layers, thus exposing older sediment and destabilizing the bed. The higher stresses also increase the amount of suspended material to be transported and deposited in other areas, which could impact benthic communities. Careful consideration of vessel traffic patterns may identify thresholds that could significantly increase the resuspension and erosion potential of vessel wake.

\subsection{Settling velocity}

The methodology used to obtain settling velocity is based on the decay in concentration over time, and the relative low error as measured by the confidence limits indicates that the results are reasonable in a statistical sense. However, the differences in settling velocity derived from three published models suggest there is a need to improve fall velocity predictions, which requires more data to develop statistically significant correlations. Average settling velocities of $0.5 \mathrm{~mm} / \mathrm{s}$ means that resuspended material distributed over a $1 \mathrm{~m}$ water column can take over 
30 min to settle out. This is enough time for tidal currents to redistribute sediment to other regions in the saltmarsh before settling. Because the source is located near the bank, the majority of the along-channel transport will remain in the shallow areas. In regions with extensive oyster cover, vessel-induced resuspension of slowly settling fine-grained particles could be a fundamental transport mechanism that causes reductions in light penetration and burial of fragile benthic communities.

\subsection{Utility of these results to other applications}

Given the generalized nature of the energy processes of wave propagation, the results presented here should translate to wakes generated by other types of vessels. While the fundamental sediment dynamic processes are similar, there are likely some scale effects due to the dominant wake type generated by larger versus smaller vessels. Larger vessels generate larger waves and release greater amounts of energy at the shoreline. Greater energy leads to higher bed shear stress and greater potential to resuspend material and to contribute to bank erosion. However, bed layering in regions with oscillatory flow implies a transition between an easily eroded surface layer and a more resistant deeper layer. Under these conditions, shear stress may have to increase substantially before eroding the compacted bed sediment that lies below the surficial layer. Higher concentrations affect water quality to a greater extent by reducing light penetration through increased scattering and absorption (Gallegos et al. 1990). However, the theoretical foundations that relate stress to concentration are universal.

The stress generation process does depend on the characteristics of the boat wake reaching shallow depths and ultimately wave breaking on the shoreline as well as bed type and layering. For example, the largest disturbances generated by commercial vessels such as container ships are the transverse stern waves (Maynord 2007). The transverse wave possesses steep slopes and large wave heights resulting in greater drawdown as the wave impinges on the shoreline. Once the wave breaks, the potential energy is transferred to kinetic energy producing a large run up. The dispersive waves are the dominant mode for smaller watercraft (recreational boats) and are the type discussed in this study. These waves generally form a wave packet with the largest waves in the center of the packet bracketed by smaller waves leading and trailing the maximum wave. As the wave propagate towards shore, the longer waves arrive first, consistent with the wavelet analysis showing the shift in peak period to 
shorter waves as the wave train passes. Linear wave theory predicts that longer waves travel faster in intermediate and deep water, which is also consistent with the measurements. These results apply equally to all types of vessel wake whether generated by recreational or commercial craft. The connection that allows for a more universal framework is tied to the vessel-wake model, which predicts the wave height and wave decay. Once the wave height and period are known, linear wave theory is used to compute the shear stresses and associated wave energy dissipation and sediment resuspension independent of the vessel type. The other factors such as bed composition, layering, and sources of ambient energy (tides, winds) are independent of the vessel type. Thus, the procedures and methodologies presented in this study can be applied to other USACE projects in which the effects of vessel wake need to be gauged in terms of ambient forcing conditions.

\subsection{Vessel wake demonstration analysis}

The methodology to predict the vessel equivalent energy dissipation contains several assumptions that are not well constrained: (1) accurate estimates of the number of waves per wave group and the corresponding wave height distribution and (2) the fraction of the tidal energy dissipation responsible for bank erosion. The first could be investigated using data similar to that presented in this report. Measurements of the number of waves per group and their distribution could be compared to vessel characteristics such as wave height near the vessel, vessel speed, and other geometric characteristics. A literature search did not find any studies reporting on the relationship between the number of waves per wave packet and vessel parameters and attempts to develop an empirical model with the present data did not produce substantial results. Furthermore, the numbers of waves per packet were available for this study, so there was no need to generate a model.

To lowest order, the individual waves in a wave group follow a Rayleigh distribution, and this has been applied to vessel wake (Didenkulova and Rodin 2013). The Rayleigh distribution requires the maximum wave and a single scale parameter. The maximum wave is predicted from the vessel wake model and the scale parameter can be determined by fitting the Rayleigh distribution to wave packet data. While this gives the distribution of waves, it cannot be applied in a generic sense unless the number of waves per packet is known. If a methodology to predict the number of waves can be applied, then the Rayleigh distribution can be used to 
establish the distribution. It is noted that using the maximum wave height to represent all waves in a wave packet, as is done here, overestimates the wave energy. However, this also provides a conservatively high estimate of the wave energy, which may be useful in applications if the result is lower than the tidal energy. In this way, the user is more assured that the impacts of vessel waves are relatively small compared to tides.

The tidal energy calculation provides an estimate of the average dissipation per unit planform area. To obtain the dissipation per unit length of channel, which is the quantity that is compared to the wave energy, the expression must be multiplied by the channel width. Studies in rivers have shown that this overestimates the energy responsible for shoreline erosion and that a more reasonable estimate is obtained by multiplying the average channel width by 0.01 to 0.05 . Maynord et al. (2008) and Hill et al. (2002) argue that only $1 \%$ to $5 \%$ of the tidal energy is dissipated in the near bank region, as losses in the middle of the channel do not contribute directly to shoreline erosion. A similar approach is adopted in this study considering that tidal creeks are geometrically similar to rivers in that they possess a high width-to-depth ratio and deepen towards the middle. Tidal creeks also meander with shallower deposits on the inside of bends similar to rivers. Studies have indicated that creek networks scale similarly as terrestrial watersheds (Novakowski et al. 2004), so they share fundamental geomorphological characteristics and may behave similarly. Even so, tidal currents reverse direction on a periodic basis whereas river flow is unidirectional. However, during maximum flood or ebb, the flow characteristics are quasi-steady, and the dissipation due to bed friction is routinely assumed to be in equilibrium with the pressure gradient. As such, the fraction of cross-channel tidal energy responsible for bank erosion is probably similar to rivers. For purposes of applying a screening level or planning tool, this assumption is more justifiable. If the estimated tidal energy dissipation error using this approach is deemed unacceptably high, then more quantitative methods such as direct field measurements can further constrain the uncertainty. 


\section{Conclusions}

This technical report details a field data collection study of vessel-generated waves in an inter-tidal saltmarsh. The purpose of the study was to investigate sediment processes associated with vessel wake in a fine-grained sediment environment with substantial tidal influence. The results help to better constrain the effects of vessel wake on shoreline erosion and water quality in saltmarshes and other shallow water bodies, which are primary research focus areas important to the USACE Civil Works mission. Measurements of flow, turbulence, wave-generated currents, suspended sediment concentration, and particle size distribution were analyzed to obtain a deeper understanding of sediment dynamics in the context of hydrodynamic forcing from vessel wake. The results were used to develop empirical equations of sediment concentration and settling velocity that were applied to an existing vessel wake model. Application of the study findings was demonstrated through calculations of the energy dissipation due to vessels and comparing the result to the equivalent tide. A procedure for determining the cumulative energy dissipation as a function of the number of vessel passages was presented, and the results used to determine the vessel wake energy equivalent that could be used to help manage recreational vessel traffic in tide-dominated coastal plain saltmarshes. Due to the generality of the underlying theoretical assumptions, the methodologies presented in this report are transferable to other systems.

Major results of this report are the following:

1. Maximum concentrations due to vessel wake and tidal currents were similar despite differences in shear stress and resuspension mechanism. The lower maximum shear stress for the wave led to similar maximum concentrations as the higher shear stress due to the tides. It was theorized that wave breaking suspended more sediment for a given maximum wave shear stress leading to similar total concentrations as the tide. Because the maximum concentration was similar for both mechanisms, it is possible that the bed consists of an easily erodible surficial layer overlying a more compact layer. The compact layer may be an artifact of the oscillatory tide in which the surface layer is reworked each tidal cycle, but the deeper layer requires a higher shear stress for the initiation of sediment motion that is not exceeded during typical tidal flow conditions. Other possible limits on 
sediment availability may be due to the presence of oysters, which anchor the bed sediment. While the explanations put forth in this report fit the data, further investigation of the actual bed erosion rate would unequivocally establish the likelihood and mechanism of bed armoring in fine-grained saltmarsh regions.

2. The amount of material resuspended due to vessel wake was regulated by tidal height in inter-tidal saltmarshes. When water level was below the height of the marsh platform, wave breaking on the exposed bank produced high concentrations of suspended sediment. Once the water level exceeded the marsh platform, the vessel waves propagated over the bank and into the marsh interior carrying the majority of the energy flux. Vessels traveling at high tide produced much lower concentrations of resuspended material despite bottom orbital velocities similar to those measured during low tide. The potential effect on shoreline erosion is likely greatly reduced for vessels traveling at high tide.

3. Settling velocities derived from the concentration measurements indicate that particles can take over 30 min to settle out. During maximum flood and ebb, tidal currents could transport material to other sections of the marsh where it would be redeposited. While the estimates varied somewhat, they generally fell within the range of three previously published settling velocity models. Vessel waves produced a finite turbulence impulse that rapidly mobilized bed material but then could not sustain sediment suspensions. Particles and flocs settled under the action of gravity making it possible to estimate settling velocity from time-series concentration measurements. Vessel wake concentration data could help to refine or develop more accurate settling velocity models using techniques described in this study.

4. A simple method that compared vessel energy dissipation to the tidal equivalent was presented. The approach was easy to implement and could be applied to recreational vessels operating in tide-dominated areas. Less well-constrained parameters were treated as random variables and thus provided a measure of uncertainty in the model's predictions. This was cited as a useful tool to quantify risk and could help inform decision makers to determine if and when more robust and time-consuming measures may be warranted. 


\section{References}

Agrawal, Y. C., and H. C. Pottsmith. 2000. "Instruments for Particle Size and Settling Velocity Observations in Sediment Transport.” Marine Geology 168: 89-114. https://doi.org/10.1016/S0025-3227(00)00044-X.

Anderson, M. E., J. M. Smith, and S. K. McKay. 2011. Wave Dissipation by Vegetation. ERDC/CHL CHETN-I-82. Vicksburg, MS: U.S. Army Engineer Research and Development Center. http://hdl.handle.net/11681/1896.

Asplund, T., and Univ. of Wisconsin. 2000. The Effects of Motorized Watercrafts on Aquatic Ecosystems. PUBL-SS948-0o. Madison, WI: Department of Natural Resources. http://greentier.wisconsin.gov/lakes/publications/documents/lakes.pdf.

Been, K., and G. Sills. 1981. "Self-Weight Consolidation of Soft Soils: An Experimental and Theoretical Study." Geotechnique 31(4): 519-535. https://doi.org/10.1680/geot.1981.31.4.519.

Bilotta, G., and R. Brazier. 2008. "Understanding the Influence of Suspended Solids on Water Quality and Aquatic Biota.” Water Research 42(12): 2849-2861. https://doi.org/10.1016/i.watres.2008.03.018.

Cox, R., R. A. Wadsworth, and A. G. Thomson. 2003. "Long-Term Changes in Salt Marsh Extent Affected by Channel Deepening in a Modified Estuary." Continental Shelf Research 23.17: 1833-1846. https://doi.org/10.1016/j.csr.2003.08.002.

Daubechies, I., 1990. "The Wavelet Transform, Time-Frequency Localization and Signal Analysis.” IEEE Transactions on Information Theory 36(5): 961-1005. https://doi.org/10.1109/18.57199.

Didenkulova, I., and A. Rodin. 2013. "A Typical Wave Wake from High-Speed Vessels: Its Group Structure and Run-Up.” Nonlinear Processes in Geophysics 20: 179-188. https://www.nonlin-processes-geophys.net/20/179/2013/npg-20-179-2013.pdf.

Gallegos, C. L., D. L. Correll, and J. W. Pierce. 1990. "Modeling Spectral Diffuse Attenuation, Absorption, and Scattering Coefficients in a Turbid Estuary." Limnology and Oceanography 35(7): 1486-1502. http://onlinelibrary.wiley.com/doi/10.4319//0.1990.35.7.1486/full.

Gibbs, R. J. 1985. "Estuarine Flocs: Their Size, Settling Velocity and Density.” Journal of Geophysical Research: Oceans 90(C2): 3249-3251. http://onlinelibrary.wiley.com/doi/10.1029/JC090iC02p03249/full.

Gibson, R., G. England, and M. Hussey. 1967. "The Theory of One-Dimensional Consolidation of Saturated Clays: 1. Finite Non-Linear Consildation of Thin Homogeneous Layers.” Geotechnique 17(3): 261-273. https://doi.org/10.1680/geot.1967.17.3.261.

Grinsted, A., J. C. Moore, and S. Jevrejeva. 2004. "Application of the Cross Wavelet Transform and Wavelet Coherence to Geophysical Time Series." Nonlinear Processes in Geophysics 11(5-6): 561-566. https://www.nonlin-processesgeophys.net/11/561/2004/. 
Hill, D. F., M. M. Beachler, and P. A. Johnson. 2002. Hydrodynamic Impacts of Commercial Jet-Boating on the Chilkat River, Alaska. Pennsylvania State University, Department of Civil and Environmental Engineering. https://www.researchgate.net/profile/David_Hill24/publication/255619863_Hydrodynamic_I mpacts_of_Commercial_JetBoating_on_the_Chilkat_River_Alaska/links/5416eb880cf2fa878ad435be.pdf.

Houser, C. 2010. "Relative Importance of Vessel-Generated and Wind Waves to Salt Marsh Erosion in a Restricted Fetch Environment." Journal of Coastal Research 26(2): 230-240. https://doi.org/10.2112/08-1084.1.

Houser, C. 2011. "Sediment Resuspension by Vessel-Generated Waves along the Savannah River, Georgia." Journal of Waterway, Port, Coastal, and Ocean Engineering 137.5 (2011): 246-257. https://doi.org/10.1061/(ASCE)WW.19435460.0000088 .

Kjerfve, B. 1986. "Circulation and Salt Flux in a Well Mixed Estuary." Physics of Shallow Estuaries and Bays, edited by J. E. Van de Kreeke, 22-29. New York: SpringerVerlag. http://onlinelibrary.wiley.com/doi/10.1029/LN016p0022/summary.

Komen, G. J., L. Cavaleri, M. Donelan, K. Hasselmann, S. Hasselmann, and P. Janssen. 1996. Dynamics and Modelling of Ocean Waves. Cambridge, MA: Cambridge University Press. http://www.cambridge.org/us/academic/subjects/earth-andenvironmental-science/oceanography-and-marine-science/dynamics-and-modelling-oceanwaves?format=PB\&isbn=9780521577816\#D8ZfphEVhcJ2z4IX.97.

Kurennoy, D., T. Soomere, and K. Parnell. 2009. "Variability in the Properties of Wakes Generated by High-Speed Ferries." Journal of Coastal Research 1(Special Issue 56): 519-523. http://www.jstor.org/stable/25737631.

Manning, A. J., and K. R. Dyer. 1999. "A Laboratory Examination of Floc Characteristics with Regard to Turbulent Shearing." Marine Geology 160(1-2): 147-170. https://doi.org/10.1016/S0025-3227(99)00013-4.

Maynord, S. 1996. Return Velocity and Drawdown in Navigable Waterways. Technical Report HL-96-7. Vicksburg, MS: U.S. Army Engineer Waterways Experiment Station. http://hdl.handle.net/11681/13178.

Maynord, S. T. 2005. "Wave Height from Planing and Semi-Planing Small Boats." River Research and Applications 21(1): 1-17. http://onlinelibrary.wiley.com/doi/10.1002/rra.803/full.

Maynord, S. T. 2007. Ship Forces on the Shoreline of the Savannah Harbor Project. ERDC/CHL TR-07-7, 141. Vicksburg, MS: U.S. Army Corps of Engineers. http://hdl.handle.net/11681/7703.

Maynord, S. T., D. S. Biedenham, C. J. Fischenich, and J. E. Zufelt. 2008. Boat-WaveInduced Bank Erosion on the Kenai River, Alaska. ERDC/CHL TR-o8-05, 129. Vicksburg, MS: U.S. Army Engineer Research and Development Center. http://hdl.handle.net/11681/8579.

Miller, G. R. 1966. “The Flux of Tidal Energy out of the Deep Oceans.” Journal of Geophysical Research 71(10): 2485-2489. http://onlinelibrary.wiley.com/doi/10.1029/JZ071i010p02485/full. 
Novakowski, K. I., R. Torres, L. R. Gardner, and G. Voulgaris. 2004. "Geomorphic Analysis of Tidal Creek Networks." Water Resources Research 40(5): 1-13. http://onlinelibrary.wiley.com/doi/10.1029/2003WR002722/full.

Osborne, P. D., and E. H. Boak. 1999. "Sediment Suspension and Morphological Response under Vessel-Generated Wave Groups: Torpedo Bay Auckland, New Zealand." Journal of Coastal Research 15(2): 388-398. http://www.jstor.org/stable/4298951.

PIANC. 2003. Guidelines for Managing Wake Wash from High-Speed Vessels. Report of Working Group 41 of the Maritme Navigation Commission. International Navigation Association, Brussels. http://www.pianc.org/2872231420.php.

Press, W. H., B. P. Flannery, S. A. Teukolsky, and W. T. Vetterling. 1989. Numerical Recipes: The Art of Scientific Computing. Cambridge: Cambridge University Press. http://jasoncantarella.com/downloads/nr_excerpt.pdf.

Soomere, T. 2007. "Nonlinear Components of Ship Wake Waves." Applied Mechanics Reviews 60(3): 120-138. http://appliedmechanicsreviews. asmedigitalcollection. asme.org/article. aspx?articleid=139872 9.

Sorensen, R. M. 1997. Prediction of Vessel-Generated Waves with Reference to Vessels Common to the Upper Mississippi River System. ENV Report 4. Rock Island: U.S. Army Corps of Engineers. http://acwc.sdp.sirsi.net/client/en_US/default/search/detailnonmodal/ent:\$002f\$002fSD_IL S\$002f0\$002fSD_ILS:108541/ada?qu=Prediction+of+vesselgenerated+waves+with+reference+to+vessels+common+to+the+upper+Mississippi+River+Syste $\underline{m \& p e}=r \% 3 A 0$.

Styles, R., and S. M. Glenn. 200o. "Modeling Stratified Wave and Current Bottom Boundary Layers on the Continental Shelf." Journal of Geophysical ResearchOceans 105(C10): 24119-24139. http://onlinelibrary.wiley.com/doi/10.1029/2000JC900115/full.

Styles, R., and S. M. Glenn. 2002. "Modeling Bottom Roughness in the Presence of WaveGenerated Ripples.” Journal of Geophysical Research-Oceans 107(C8): 24-21, 24-15. http://onlinelibrary.wiley.com/doi/10.1029/2001JC000864/full.

Thompson, W. L. K. 1887. "On the Waves Produced by a Single Impulse in Water of Any Depth, or in a Dispersive Medium.” In Proceedings of the Royal Society of London, London, 409-433. http://rspl.royalsocietypublishing.org/content/42/251257/80.full.pdf.

Torres, R., and R. Styles. 2007. "Effects of Topographic Structure on Salt Marsh Currents." Journal of Geophysical Research: Earth Surface 112(F2). http://onlinelibrary.wiley.com/doi/10.1029/2006JF000508/full.

Traynum, S., and R. Styles. 2007. "Flow, Stress, and Sediment Resuspension in a Shallow Tidal Channel." Estuaries and Coasts 30(1): 94-101. https://link.springer.com/article/10.1007/BF02782970. 
Voulgaris, G., and S. T. Meyers. 2004. "Temporal Variability of Hydrodynamics, Sediment Concentration and Sediment Settling Velocity in a Tidal Creek." Continental Shelf Research 24(15): 1659-1683. https://doi.org/10.1016/i.csr.2004.05.006.

Wargo, C. A., and R. Styles. 2007. "Along Channel Flow and Sediment Dynamics at North Inlet, South Carolina.” Estuarine, Coastal and Shelf Science 71(3-4): 669-682. https://doi.org/10.1016/i.ecss.2006.09.016.

Weggel, J. R., and R. M. Sorensen. 1986. "Ship Wave Prediction for Port and Channel Design.” Ports 86. http://cedb.asce.org/CEDBsearch/record.jsp?dockey=0048454.

Whitman, E. R., and M. A. Reidenbach. 2012. "Benthic Flow Environments Affect Recruitment of Crassostrea virginica Larvae to an Intertidal Oyster Reef." Marine Ecology Progress Series 463: 177-191. https://doi.org/10.3354/meps09882.

Winterwerp, J. C. 1998. "A Simple Model for Turbulence Induced Flocculation of Cohesive Sediment." Journal of Hydraulic Research 36(3): 309-326. https://doi.org/10.1080/00221689809498621.

Winterwerp, J. C., and W. G. Van Kesteren. 2004. Introduction to the Physics of Cohesive Sediment Dynamics in the Marine Environment. Amsterdam, Netherlands: Elsevier. https://www.elsevier.com/books/introduction-to-the-physics-of-cohesive-sedimentdynamics-in-the-marine-environment/winterwerp/978-0-444-51553-7. 


\section{Appendix A: Vessel Wake Plots}

Time series of velocity and concentration for each burst with vessel wake is presented in the next nine figures. The upper plot identifies the burst with respect to the local tidal phase. Tidal height is obtained from a water level gauge maintained by the Baruch Marine Field Station located at North Inlet. The time series includes the along channel and cross-channel components of velocity as well as the suspended sediment concentration from the ADV backscatter and the LISST. The LISST concentration is computed by summing the values of the 32 bins to obtain the total suspended load. 
The first event includes two vessel wake passages (Figure A.1).

Concentrations increase in the wake of the large velocity amplitudes caused by vessel generated waves. Saturation of the LISST due to high concentrations leads to complete attenuation of the laser beam and a drop in concentration to zero during the most energetic period of sediment mixing. Concentrations prior to the vessel passage are around $40 \mathrm{mg} / \mathrm{L}$. By the end of the burst, concentrations have almost reduced to background levels.

Figure A.1. First burst in which two vessel wake events occurred.

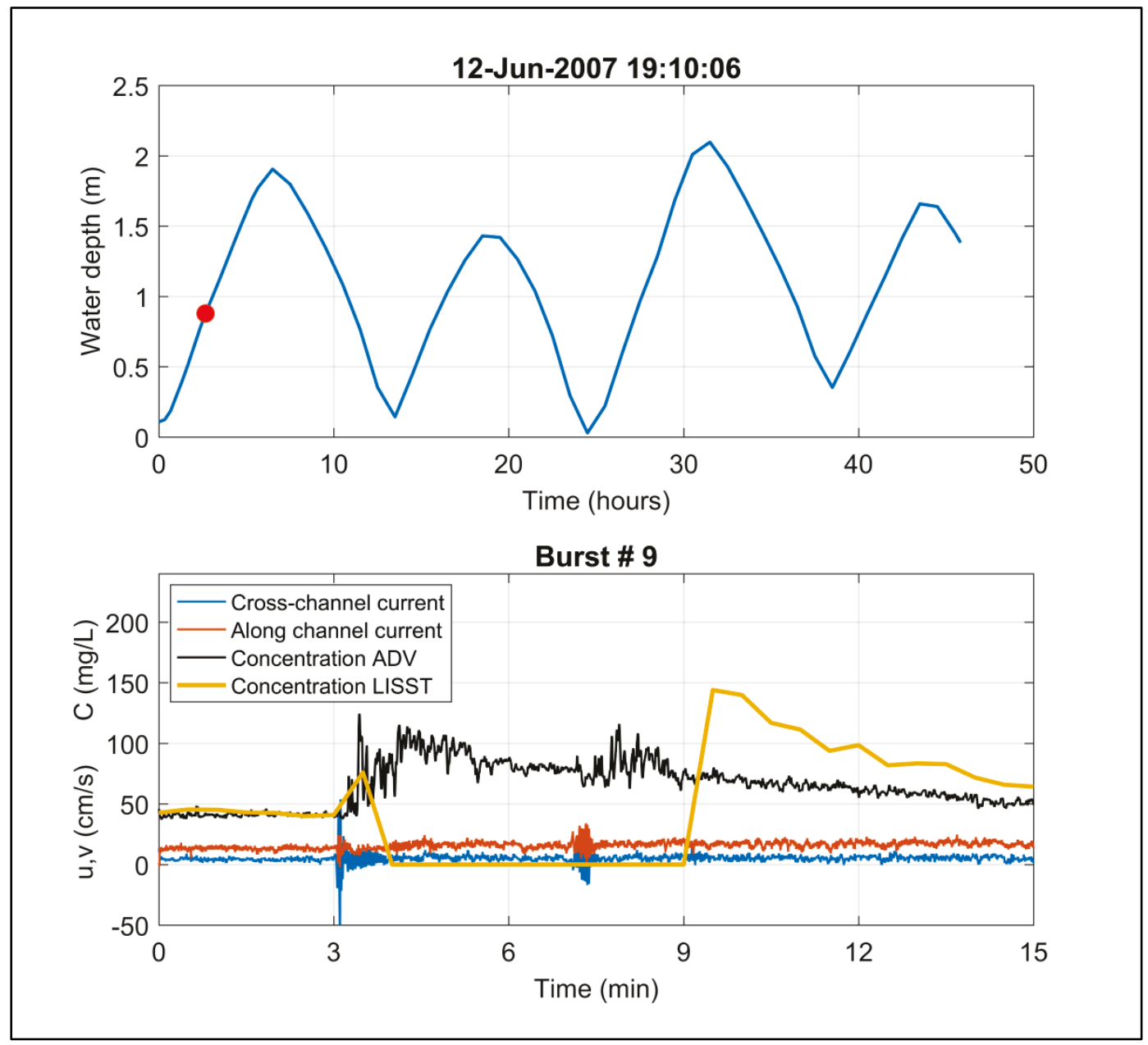


The second burst is depicted in Figure A.2. In this case, four individual wake events occurred between 5 and $9 \mathrm{~min}$. Concentration increases after the first vessel passage and remains elevated until after the final vessel passage. There are a few localized increases in concentration following the latter bursts. Concentrations then decay for the remainder of the burst.

Figure A.2. Second burst with four vessel wake events.

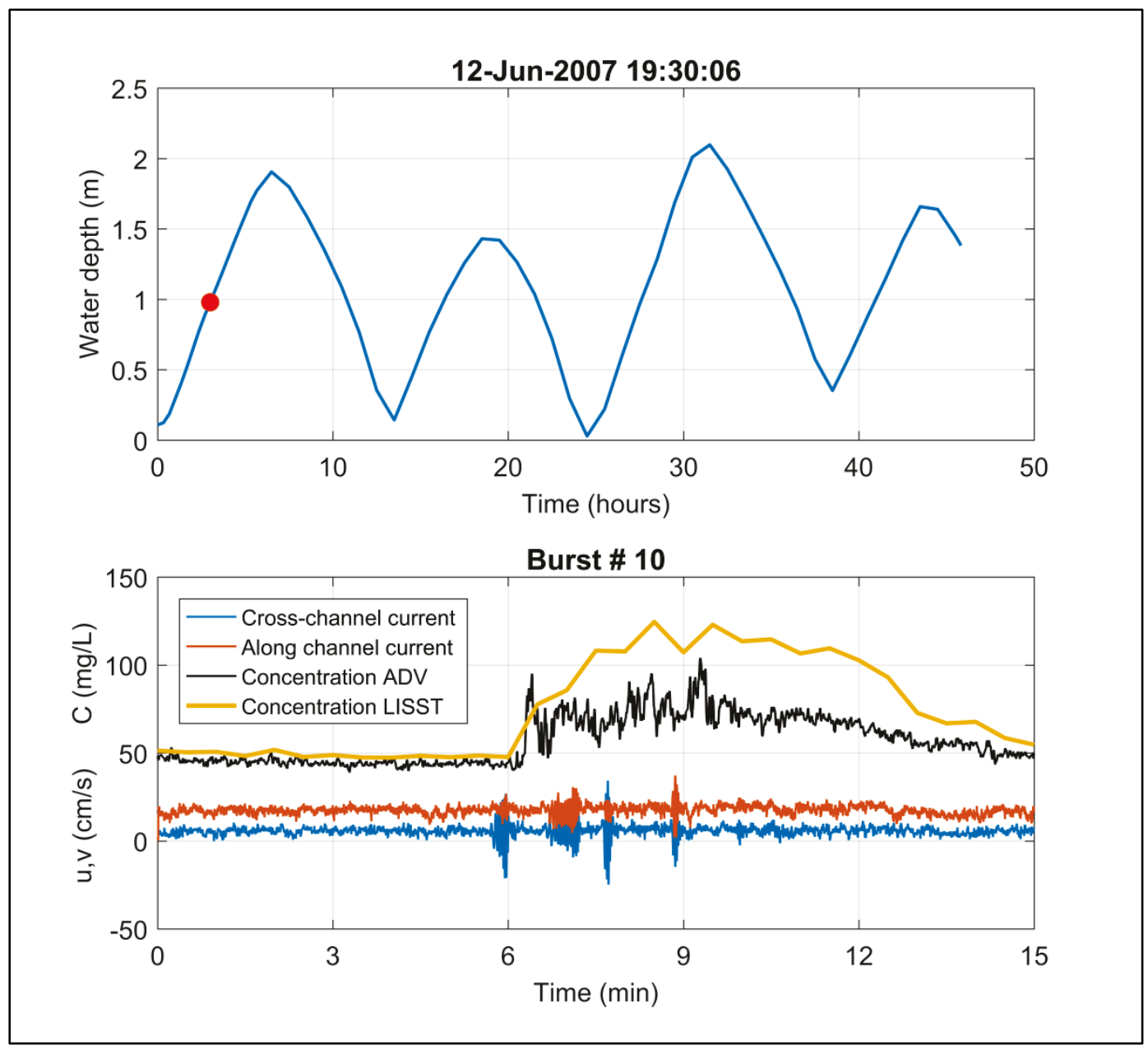


The third burst is depicted in Figure A.3. This burst had three individual vessel wake events at approximately 6,10 and $12 \mathrm{~min}$. Concentrations increase after the first burst and then begin to decay. Small increases in concentration occur after the second and third bursts but do not produce as high of concentrations compared to the first event.

Figure A.3. Third burst with three vessel wake events.

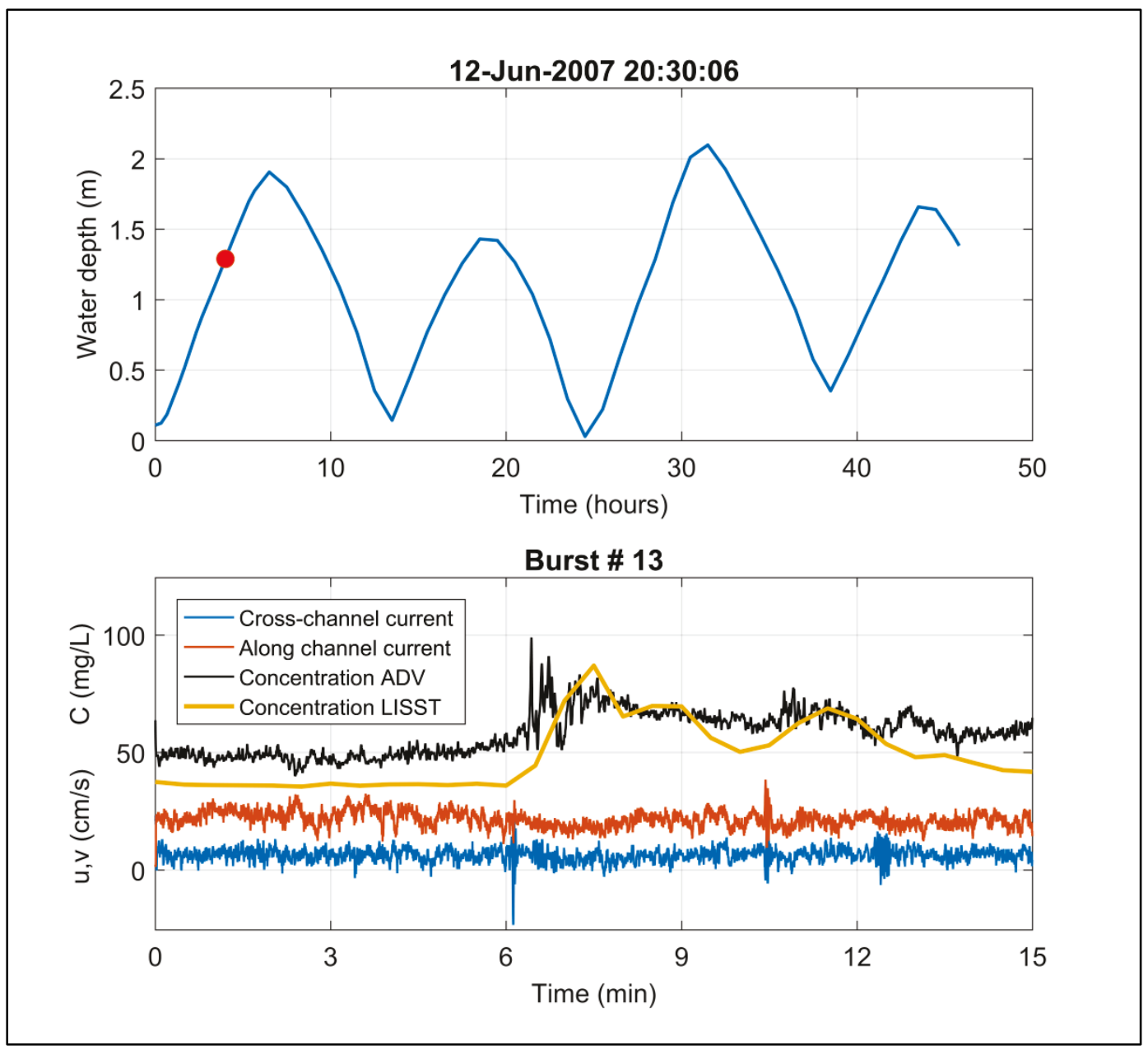


The fourth burst is depicted in Figure A.4. This burst had four vessel passages. The LISST shows lower average concentrations compared to the $\mathrm{ADV}$. While the LISST may be responding to the second and third passage, it is difficult to correlate increases in concentration to vessel wake for the $\mathrm{ADV}$. Part of this may be due to the fact that the average background concentration is $80 \mathrm{mg} / \mathrm{L}$. The ambient forcing is producing high concentrations so that the added effect of the waves is negligible. This is consistent with a sediment threshold in which the surface layer is in suspension but the deeper layers remain immobile. The water depth is over the marsh bank so that the vessel waves propagate into the marsh interior and do not break on the bank. This may reduce suspensions due to waves as well.

Figure A.4. Fourth burst with vessel wake.

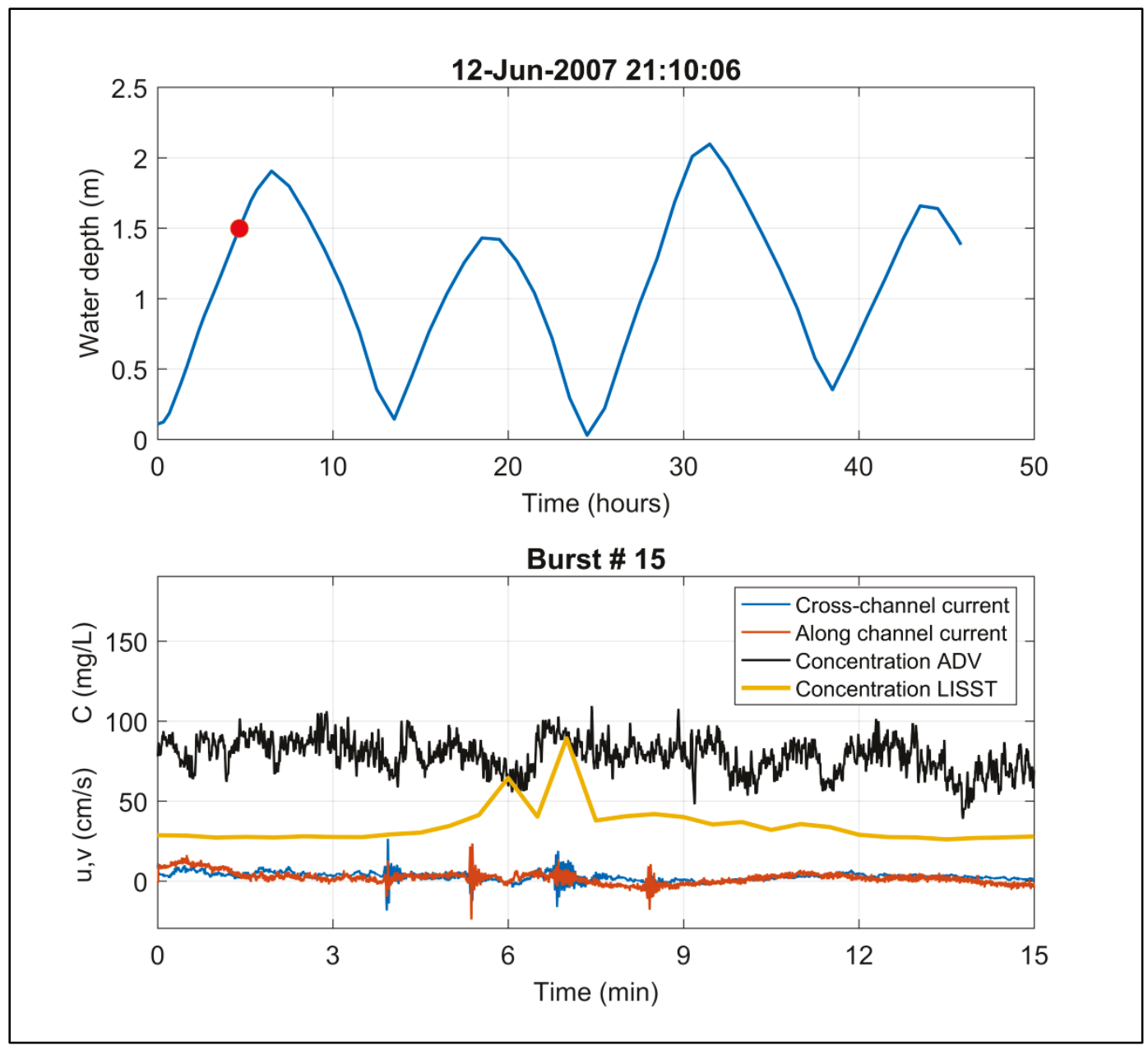


The fifth burst with vessel wake is depicted in Figure A.5. The concentration for the LISST is lower than the ADV. Fluctuations in the concentration before the first vessel passage make it difficult to determine if there is a noticeable response due to vessel wake. Average background concentrations are approximately $75 \mathrm{mg} / \mathrm{L}$, which is still relatively high. Like the previous burst, the water depth exceeds the marsh platform elevation so that the waves propagate into the marsh interior.

Figure A.5. Fifth burst with vessel wake.

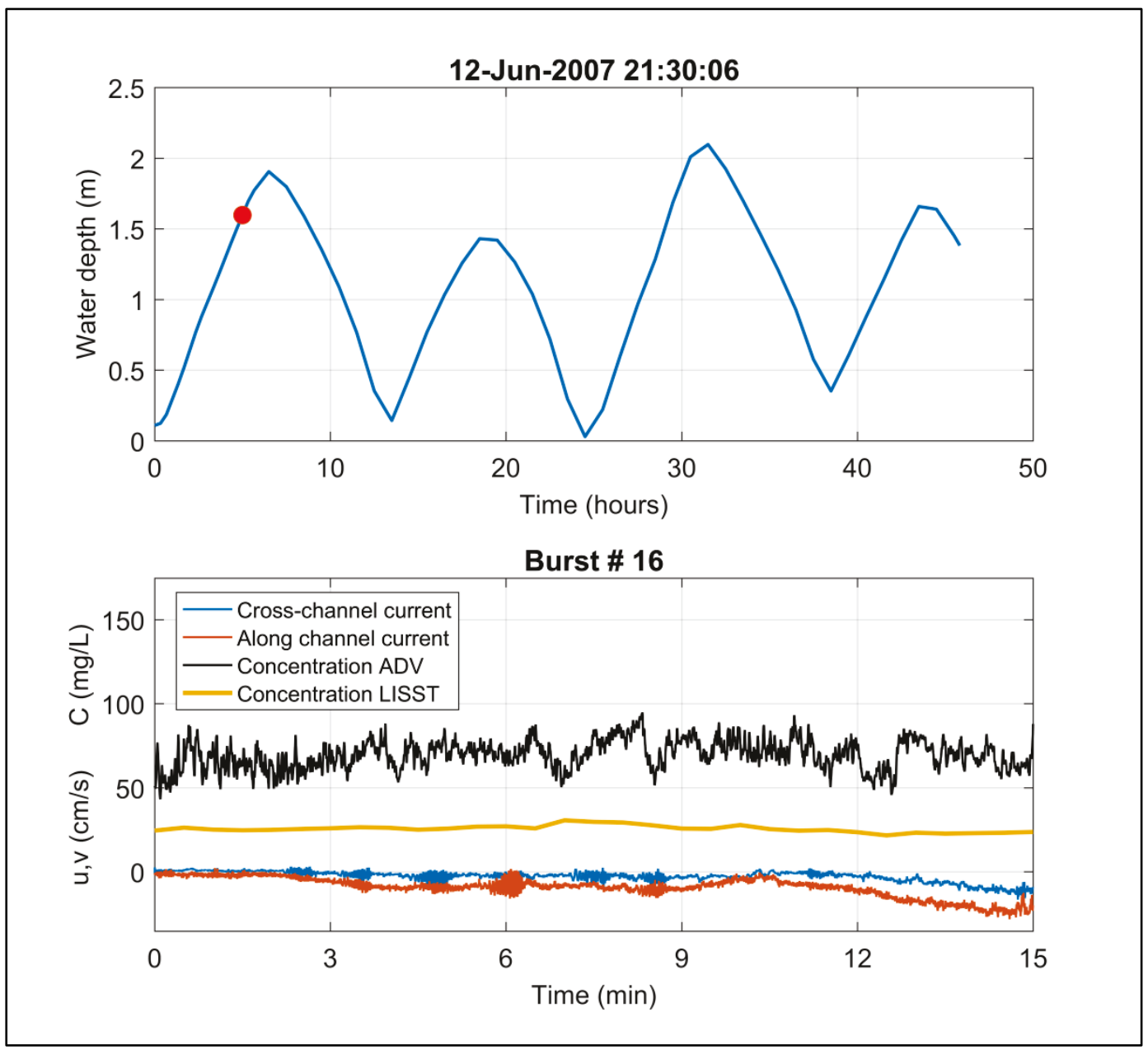


The sixth burst with waves is depicted in Figure A.6. The four vessel passages occur on the falling limb of the tide, and there is a clear correlation between vessel wake and concentration. Note the background concentration is $50 \mathrm{mg} / \mathrm{L}$, which is lower than the previous two bursts that showed no, or little, correlation between concentration and vessel wake. The LISST becomes saturated as indicated by the drop in concentration after the second vessel passage.

Figure A.6. Sixth burst with vessel wake and the last burst over the oysters.

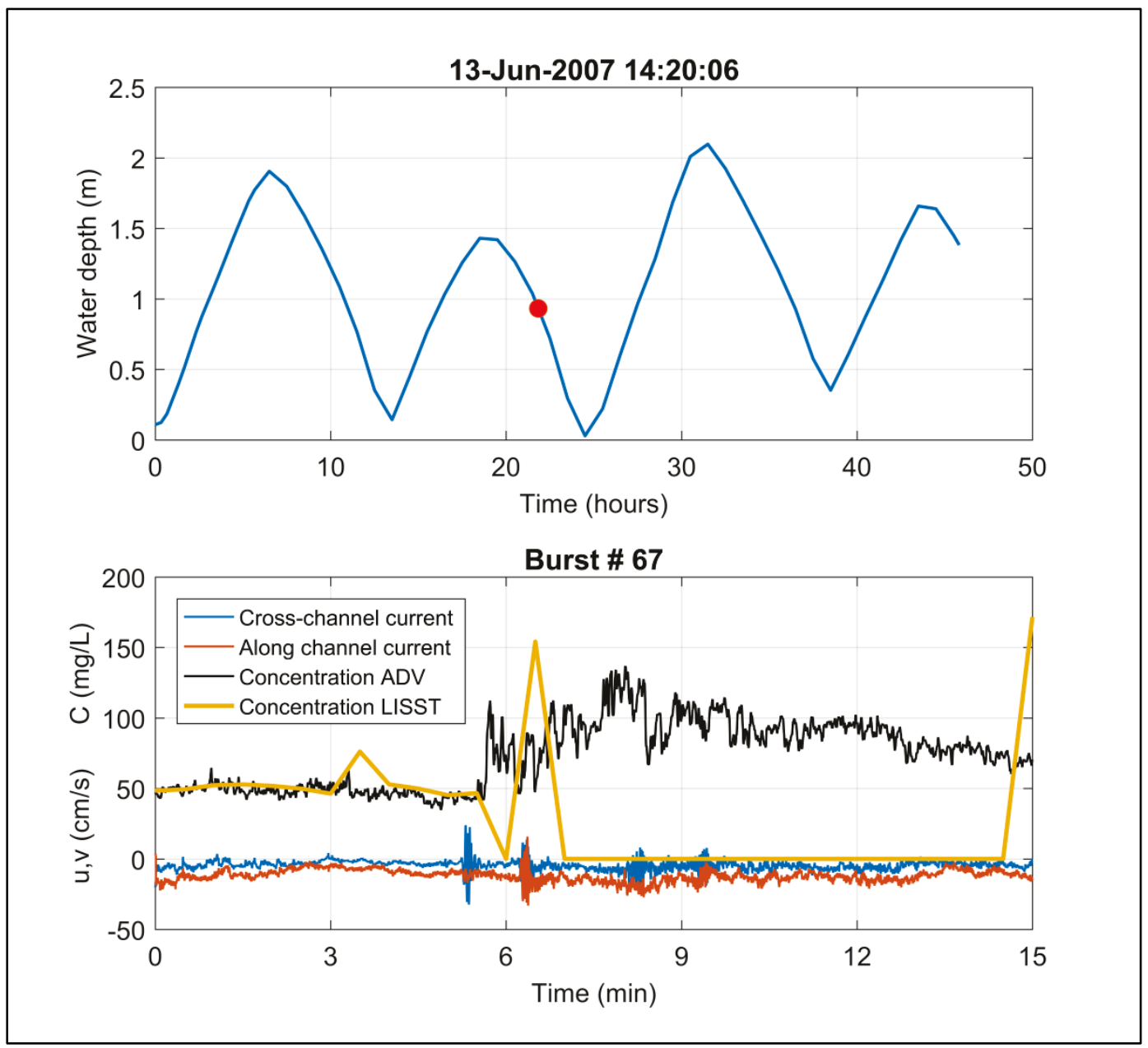


The seventh burst with waves is depicted in Figure A.7. The vessel passage occurs near low tide, and this represents the first case over the mud bank. Maximum concentrations are highest for this burst. The LISST also becomes saturated after the vessel passage.

Figure A.7. Seventh burst with vessel wake. This is the first burst over the mud bank.

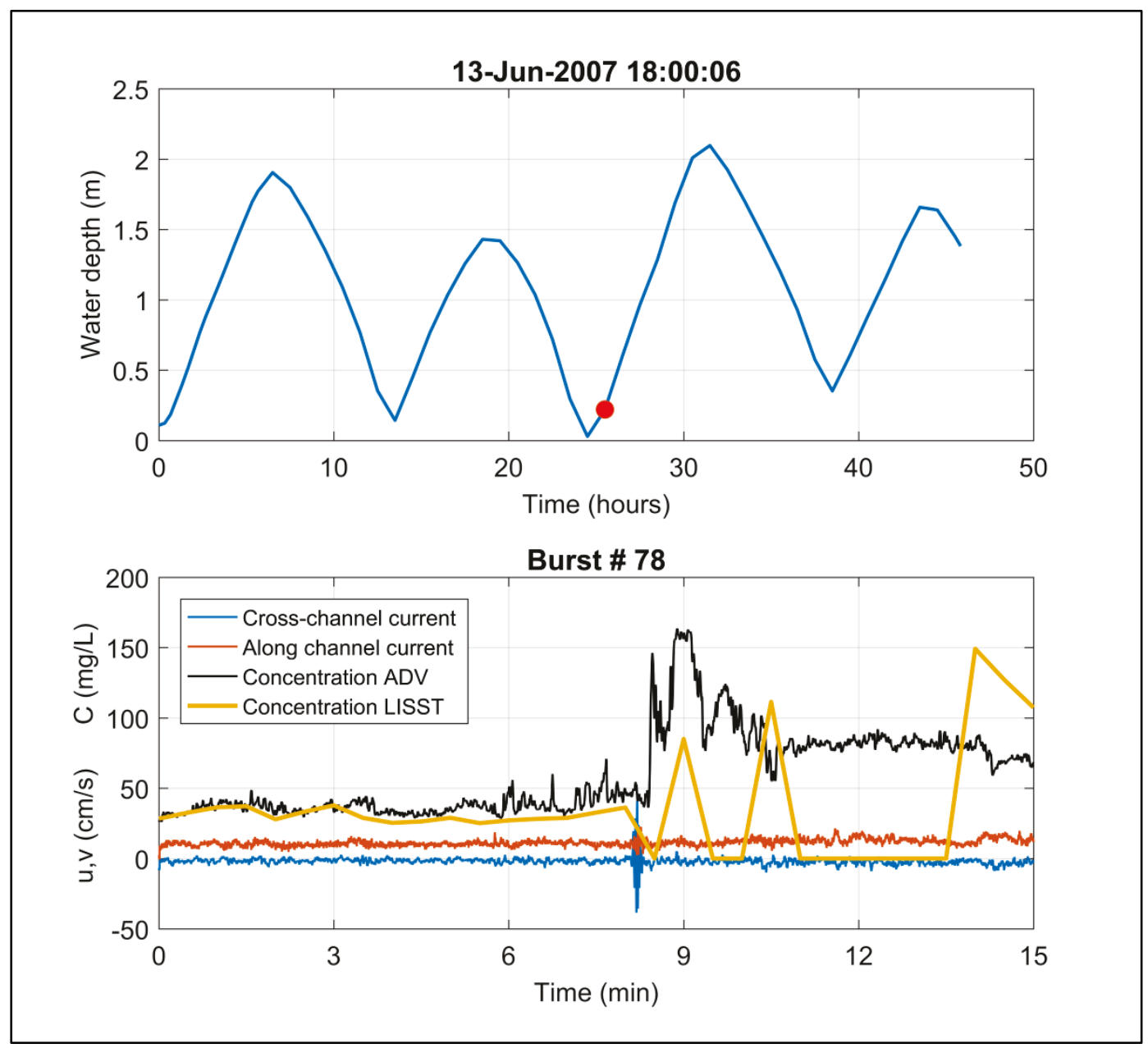


The eight burst with waves is depicted in Figure A.8. The vessel passage occurs near low tide, and this represents the second case over the mud bank. There is a clear increase in concentration following the vessel passage, with a corresponding drop in the LISST due to complete attenuation of the laser.

Figure A.8. Eighth burst with vessel wake.

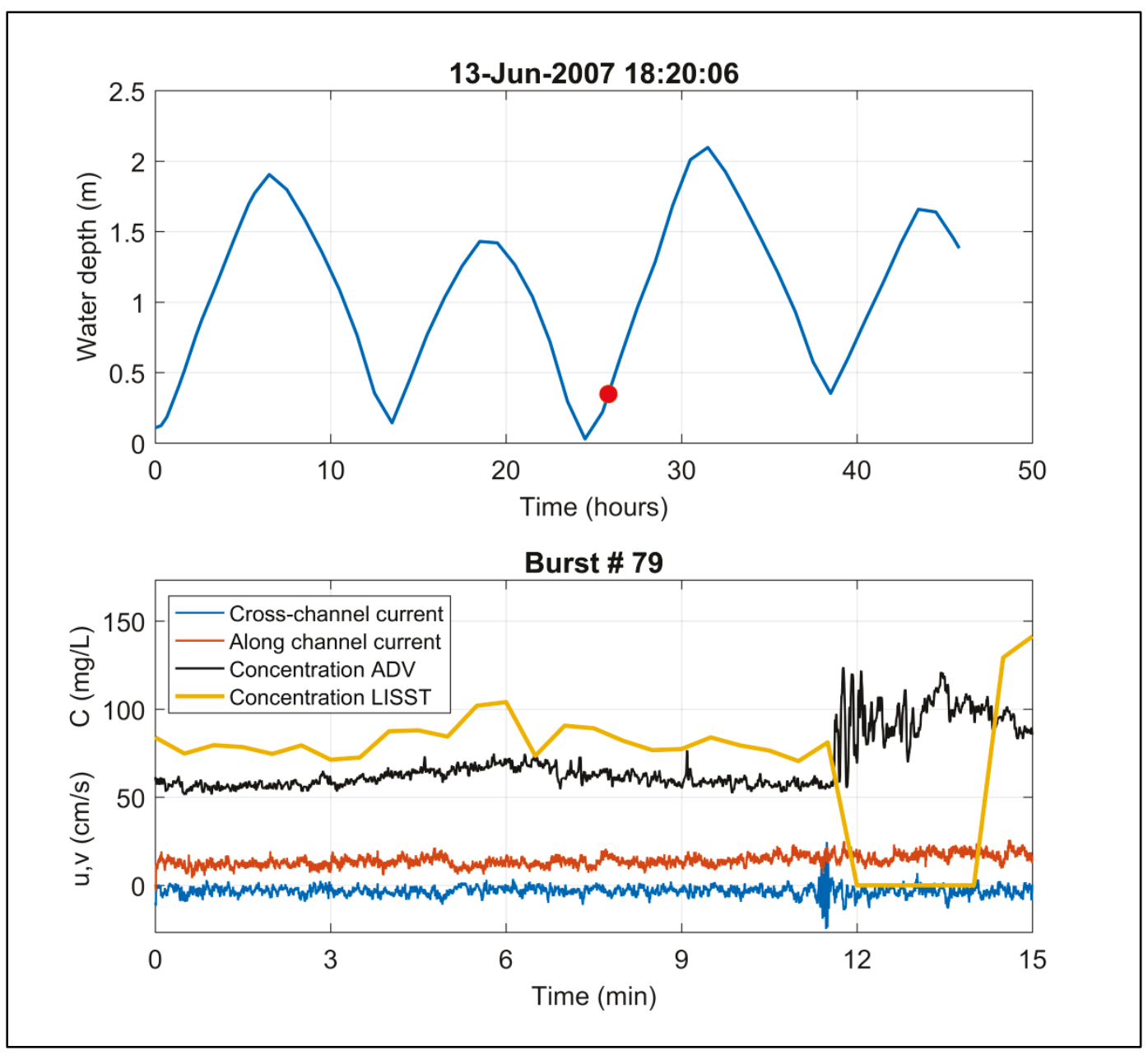


The ninth burst with waves is depicted in Figure A.9. The vessel passage occurs near low tide, and this represents the final case over the mud bank and the field study. There is some response to the vessel passage, but the concentrations are reduced. This is likely due to the fact that the water is getting deeper and the wave velocities are more attenuated at the bottom.

Figure A.9. Final burst with vessel wake.

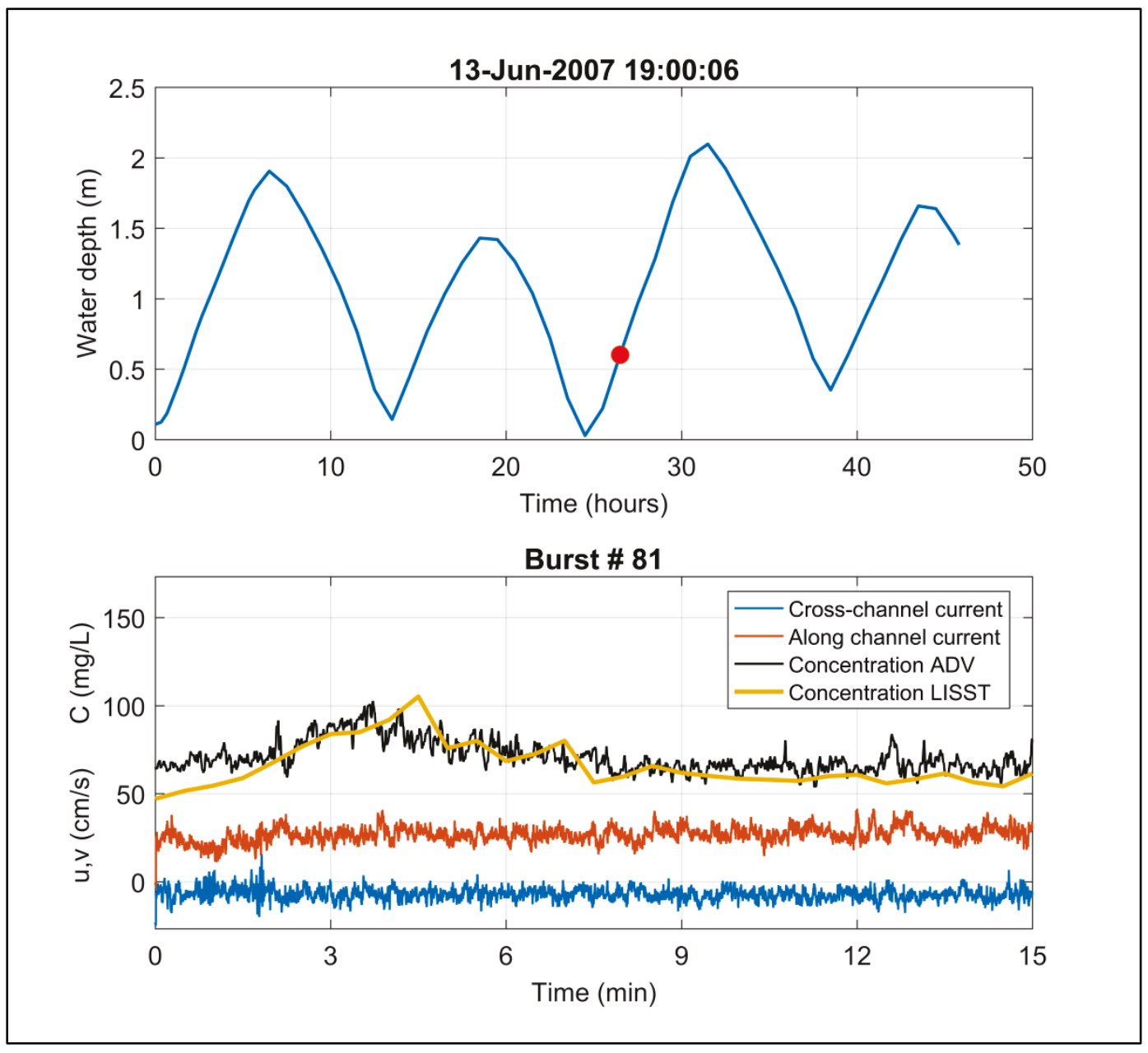




\section{Appendix B: Vessel Wake Parameters}

Vessel wake parameters are derived from N246 located $0.22 \mathrm{~m}$ above the bed. Time denotes the start of each burst measured from the beginning of the deployment. Depth is the mean water depth for each burst. Wave height $\left(H_{m x}\right)$ is computed using linear wave theory based on bottom orbital velocity $\left(U_{b}\right)$ and wave period $(T)$ measured by the ADV.

Table B.1. Measured vessel wake parameters.

\begin{tabular}{|c|c|c|c|c|c|c|}
\hline $\begin{array}{c}\text { Time } \\
\text { (hr) }\end{array}$ & Burst \# & $\begin{array}{c}\text { Depth } \\
\text { (m) }\end{array}$ & $\begin{array}{c}\mathrm{U}_{\mathrm{r}} \\
(\mathrm{cm} / \mathrm{s})\end{array}$ & $\begin{array}{c}T \\
\text { (s) }\end{array}$ & $\begin{array}{c}U_{\mathrm{b}} \\
(\mathrm{cm} / \mathrm{s})\end{array}$ & $\begin{array}{l}\mathrm{H}_{\mathrm{mx}} \\
(\mathrm{cm})\end{array}$ \\
\hline 2.67 & 9 & 0.56 & 16.61 & 1.97 & 47.31 & 23.76 \\
\hline 2.67 & 9 & 0.56 & 16.61 & 2.06 & 24.42 & 12.22 \\
\hline 3.00 & 10 & 0.67 & 18.35 & 1.94 & 23.33 & 13.65 \\
\hline 3.00 & 10 & 0.67 & 18.35 & 2.20 & 17.76 & 10.11 \\
\hline 3.01 & 10 & 0.67 & 18.35 & 2.09 & 27.06 & 15.56 \\
\hline 3.01 & 10 & 0.67 & 18.35 & 1.94 & 23.10 & 13.51 \\
\hline 4.00 & 13 & 0.92 & 22.42 & 2.00 & 26.05 & 20.61 \\
\hline 4.01 & 13 & 0.92 & 22.42 & 1.97 & 20.09 & 16.03 \\
\hline 4.01 & 13 & 0.92 & 22.42 & 1.89 & 12.39 & 10.17 \\
\hline 4.67 & 15 & 1.02 & 3.35 & 2.57 & 24.21 & 18.64 \\
\hline 4.67 & 15 & 1.02 & 3.35 & 2.35 & 29.07 & 23.27 \\
\hline 4.67 & 15 & 1.02 & 3.35 & 2.40 & 20.81 & 16.50 \\
\hline 4.67 & 15 & 1.02 & 3.35 & 1.96 & 14.49 & 12.97 \\
\hline 5.00 & 16 & 1.10 & 9.56 & 1.67 & 3.85 & 4.49 \\
\hline 5.00 & 16 & 1.10 & 9.56 & 1.83 & 6.30 & 6.58 \\
\hline 5.00 & 16 & 1.10 & 9.56 & 2.05 & 6.18 & 5.80 \\
\hline 5.00 & 16 & 1.10 & 9.56 & 2.80 & 10.26 & 8.10 \\
\hline 5.00 & 16 & 1.10 & 9.56 & 2.46 & 7.28 & 6.07 \\
\hline 5.01 & 16 & 1.10 & 9.56 & 2.07 & 7.39 & 6.88 \\
\hline 5.01 & 16 & 1.10 & 9.56 & 1.67 & 2.70 & 3.15 \\
\hline 21.84 & 67 & 0.75 & 12.81 & 1.89 & 24.85 & 16.37 \\
\hline 21.84 & 67 & 0.75 & 12.81 & 1.78 & 29.61 & 20.01 \\
\hline 21.84 & 67 & 0.75 & 12.81 & 1.68 & 14.03 & 9.75 \\
\hline 21.84 & 67 & 0.75 & 12.81 & 1.66 & 14.57 & 10.20 \\
\hline 25.51 & 78 & 0.33 & 11.51 & 1.88 & 31.35 & 9.79 \\
\hline 25.84 & 79 & 0.39 & 14.51 & 1.94 & 22.49 & 8.28 \\
\hline 26.50 & 81 & 0.53 & 27.74 & 2.08 & 11.54 & 5.47 \\
\hline 26.50 & 81 & 0.53 & 27.74 & 1.75 & 18.51 & 8.83 \\
\hline
\end{tabular}




\section{Appendix C: Wavelet Analysis}

Wavelet analysis for each burst with vessels is depicted in the next nine figures (C.1 - C.9). The top panel shows the wavelet spectrum and the bottom is the original high-pass filtered, cross-channel current time series to help identify individual wake events.

Figure C.1. Wavelet analysis for the first burst with vessels.

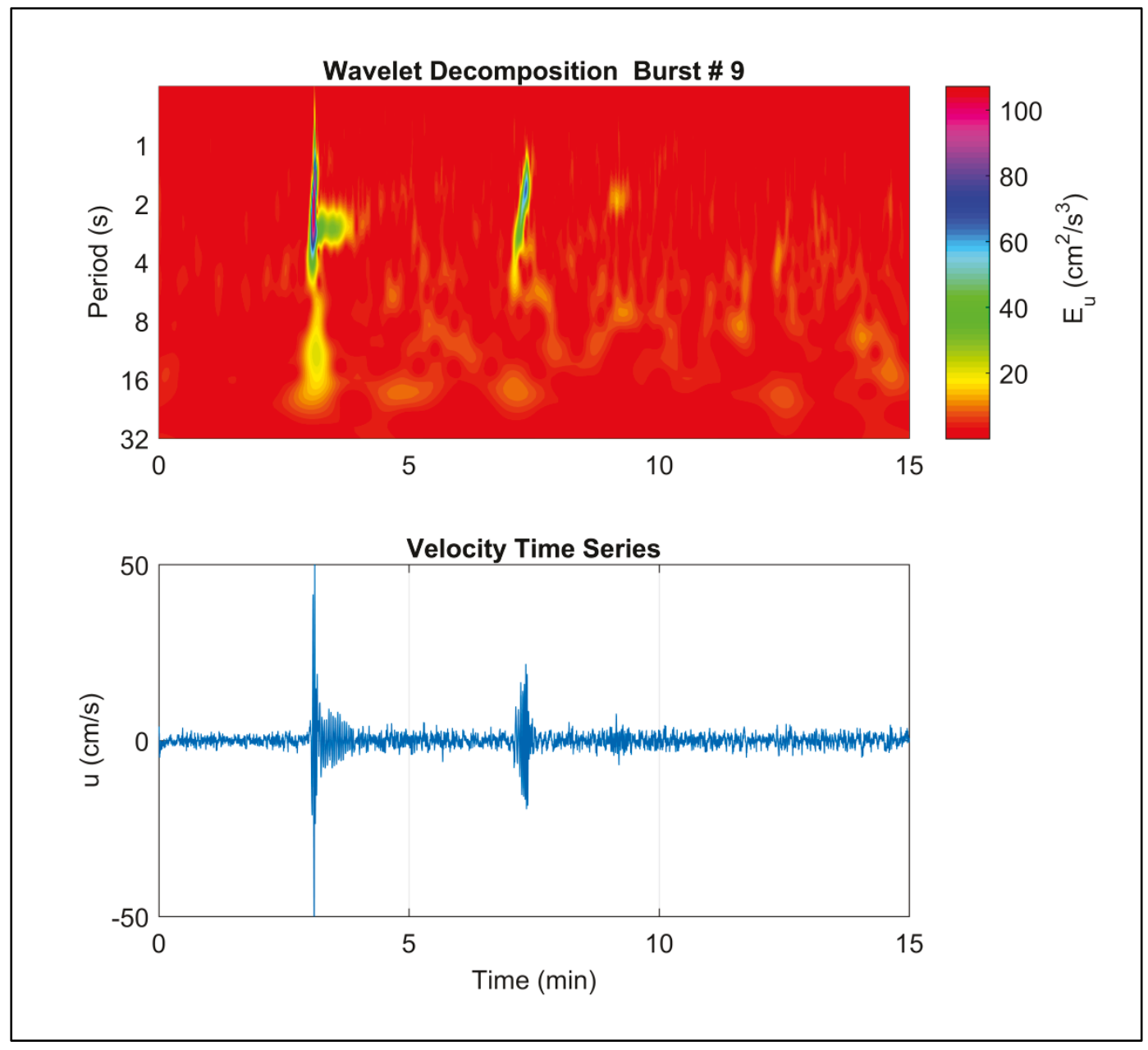


Figure C.2. Wavelet analysis for the second burst with waves. The second wave event indicates the largest shift in period.

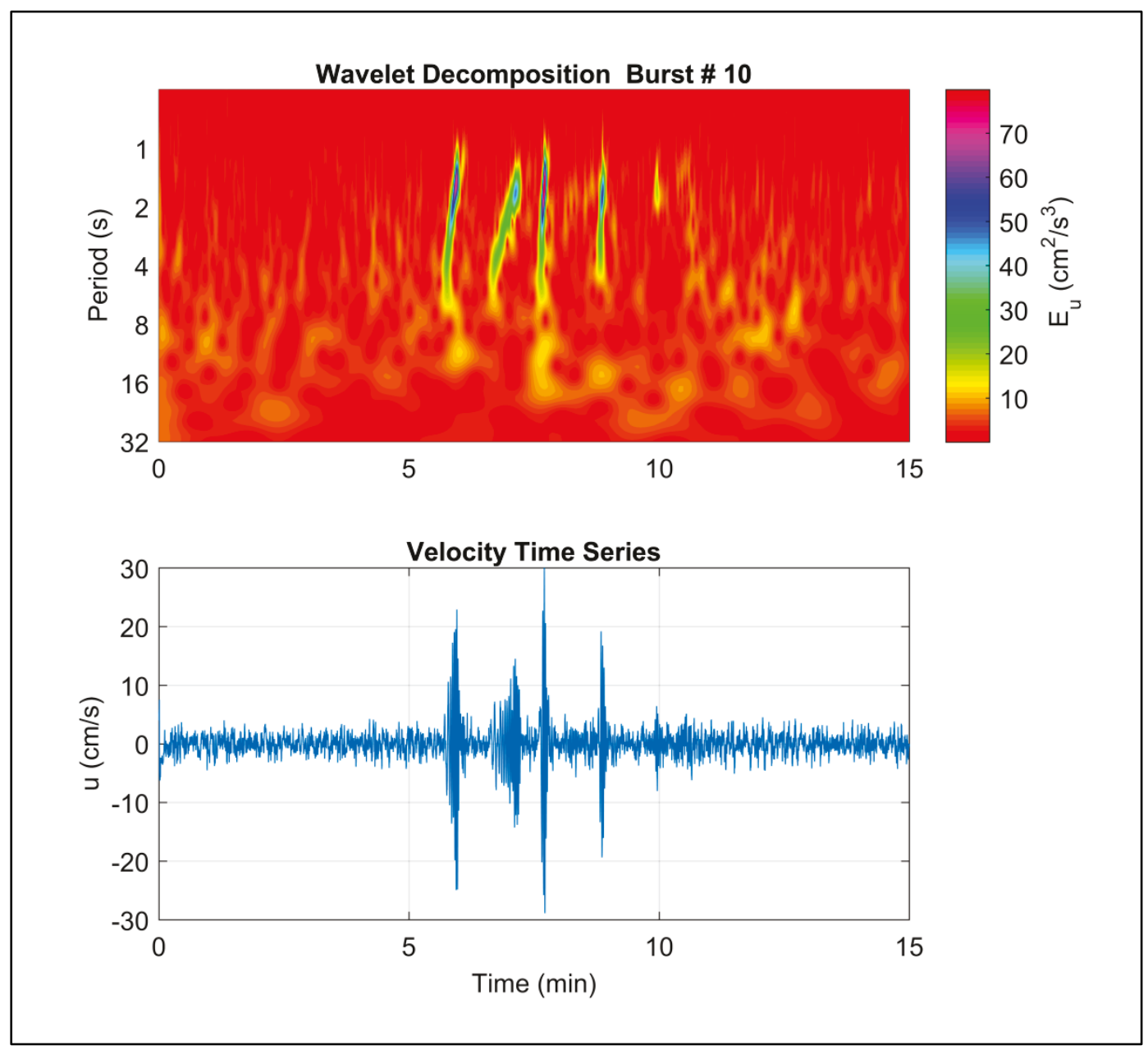


Figure C.3. Wavelet analysis for third burst with waves. The mean along channel current is the highest for the first two tidal cycles. The increase in energy density in the 4 to $16 \mathrm{~s}$ band is due to the added turbulence.

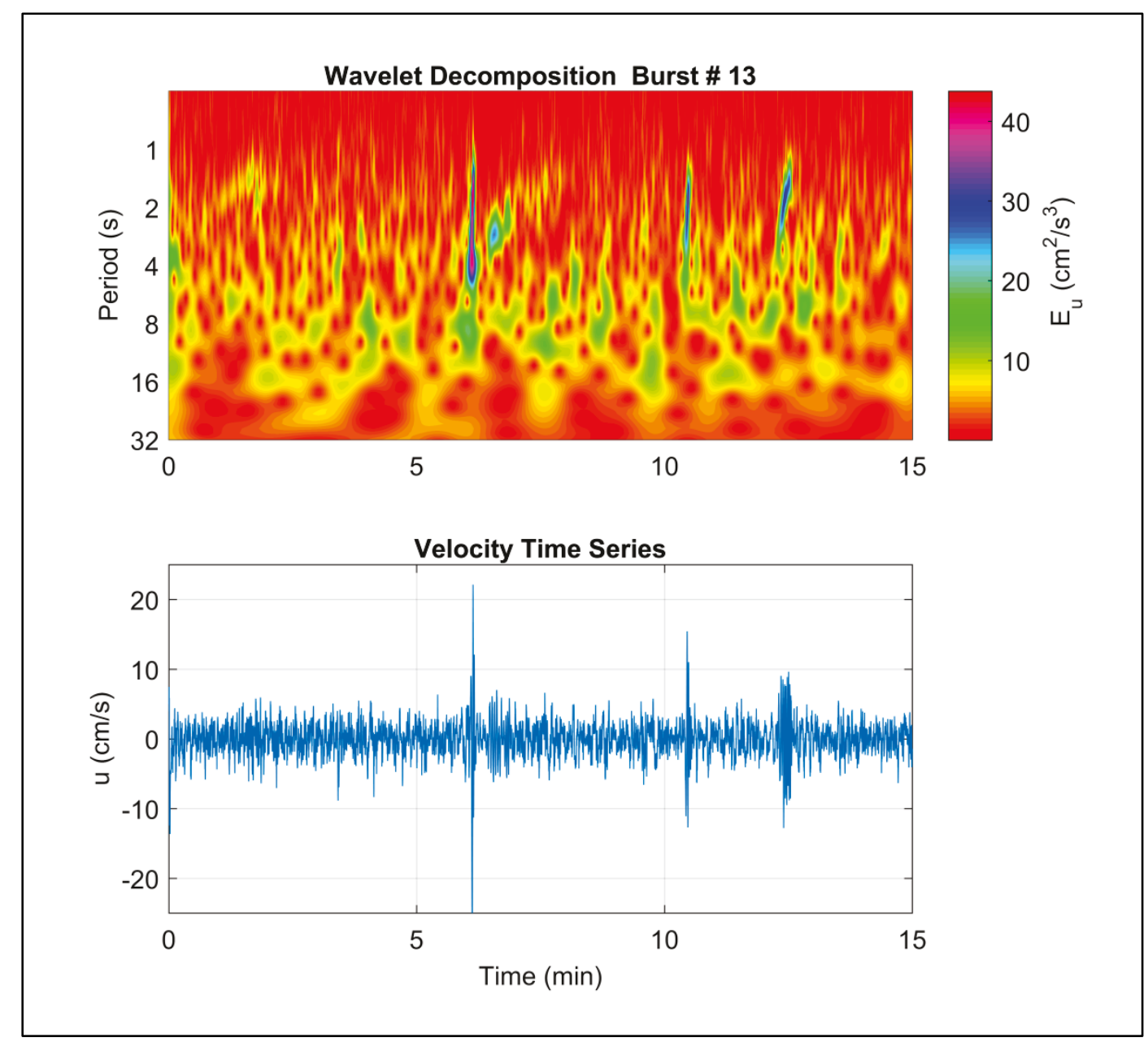


Figure C.4. Wavelet analysis for the fourth burst with waves. The mean along channel current is reduced, and there is a corresponding reduction in the energy density except for the vessel wake.

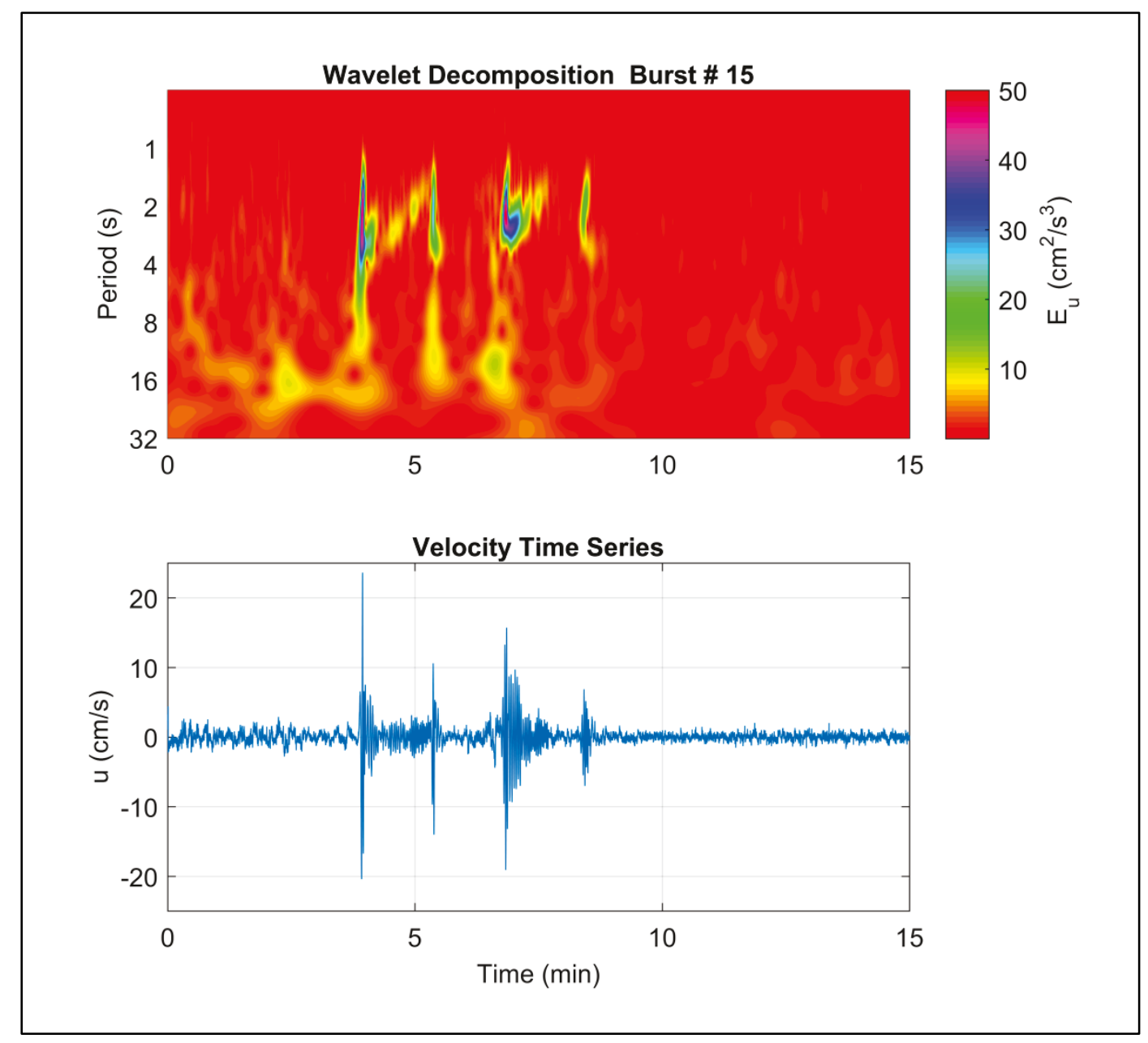


Figure C.5. Wavelet analysis for the fifth burst with waves. The multiple vessel wake events leads to residual energy bands in the 8 to $16 \mathrm{~s}$ range that persist after the last wake has passed. Maximum orbital velocities are relatively low $(5 \mathrm{~cm} / \mathrm{s})$, so the energy from waves is small.
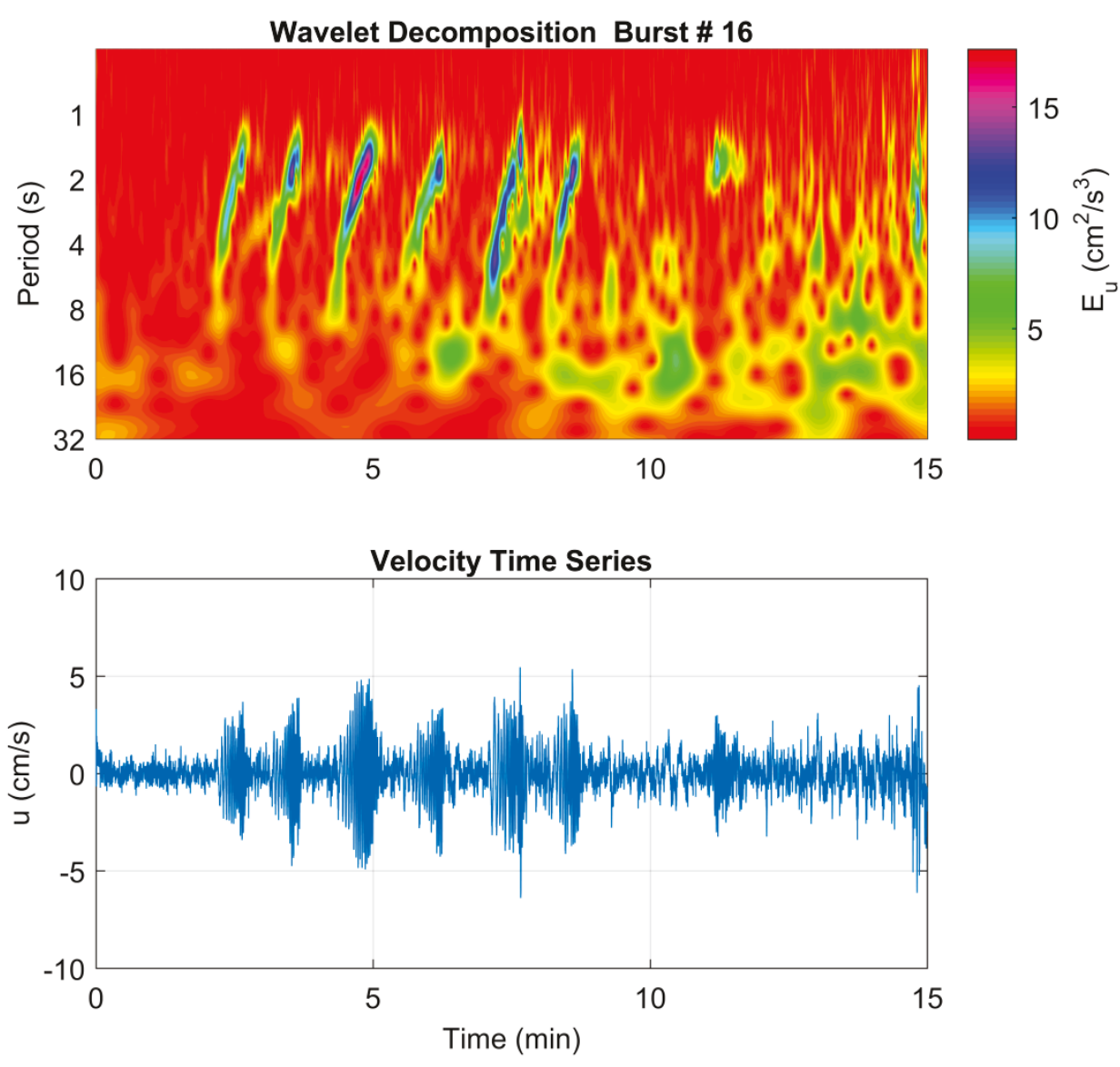
Figure C.6. Wavelet analysis for the sixth burst with waves. Maximum orbital velocities are relatively large. There are residual energy pockets at different frequencies that form in the wake of the last vessel passage. However, their energy is small compared to the vessel waves.

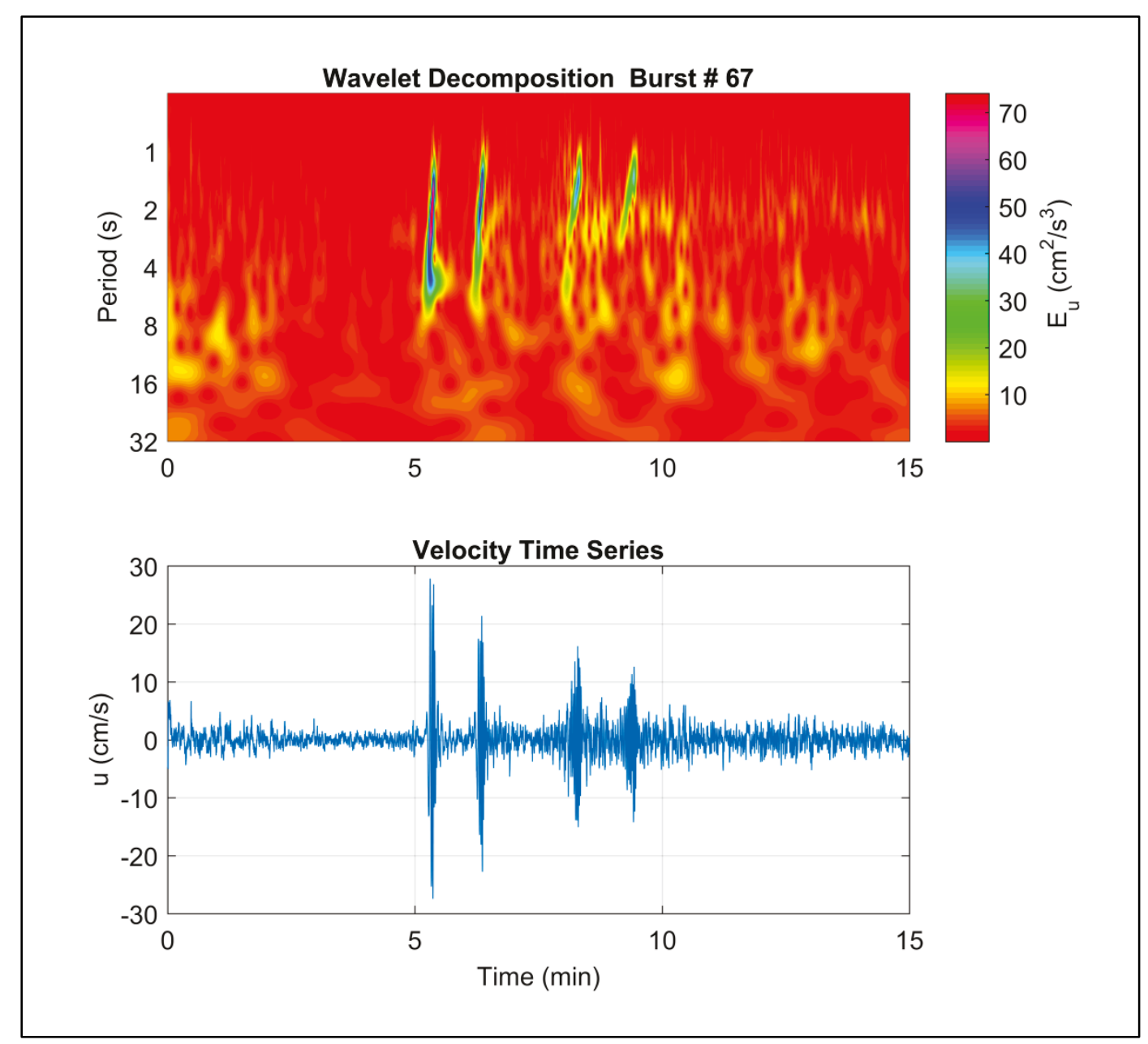


Figure C.7. Wavelet analysis for seventh burst with vessels. The energy density is narrow banded and confined to a small temporal window. There is very little residual energy pulse following the vessel wake event.

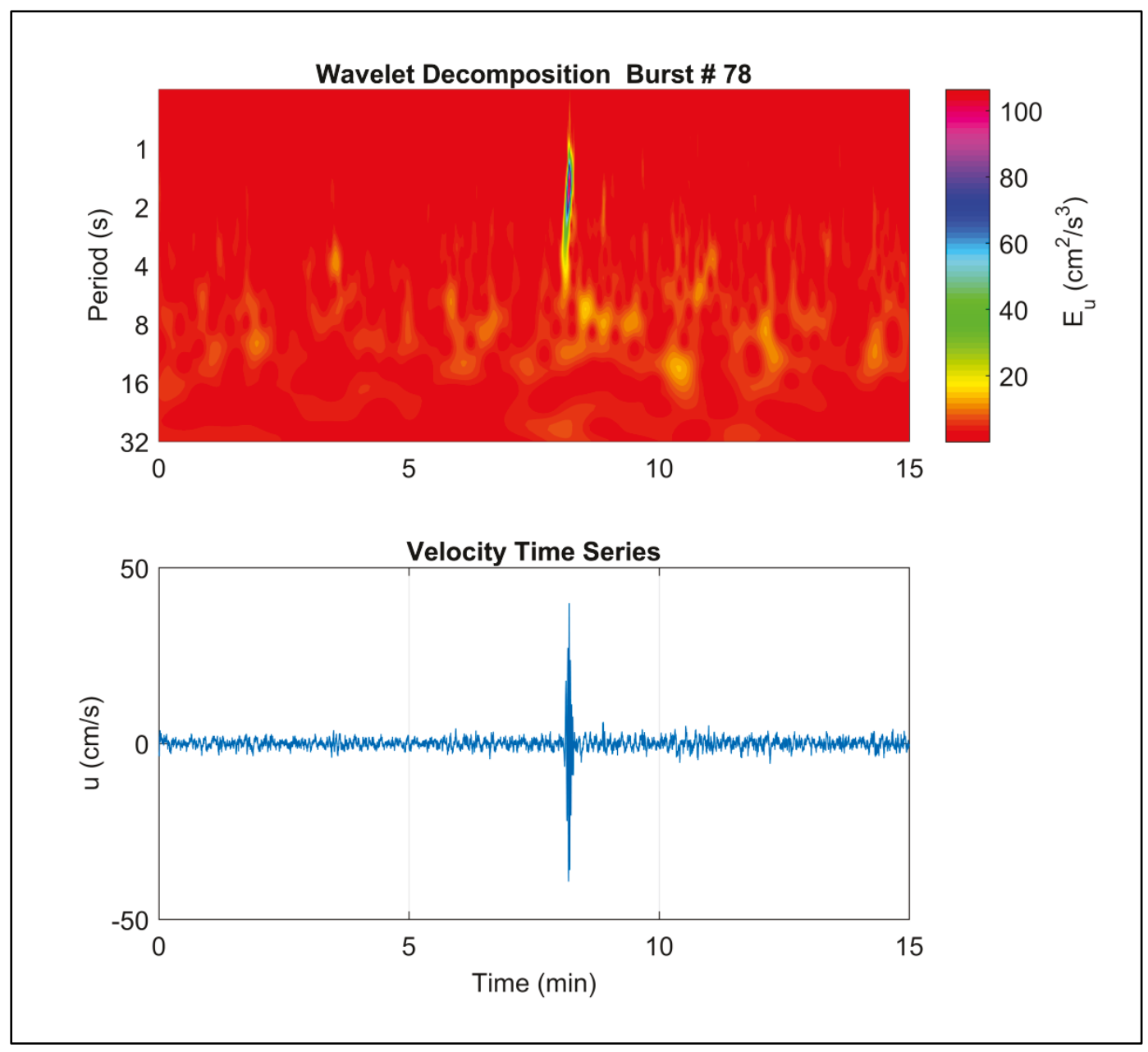


Figure C.8. Wavelet analysis for the eighth burst with vessels. Energy density is narrow banded with some residual energy pockets following the wake event.

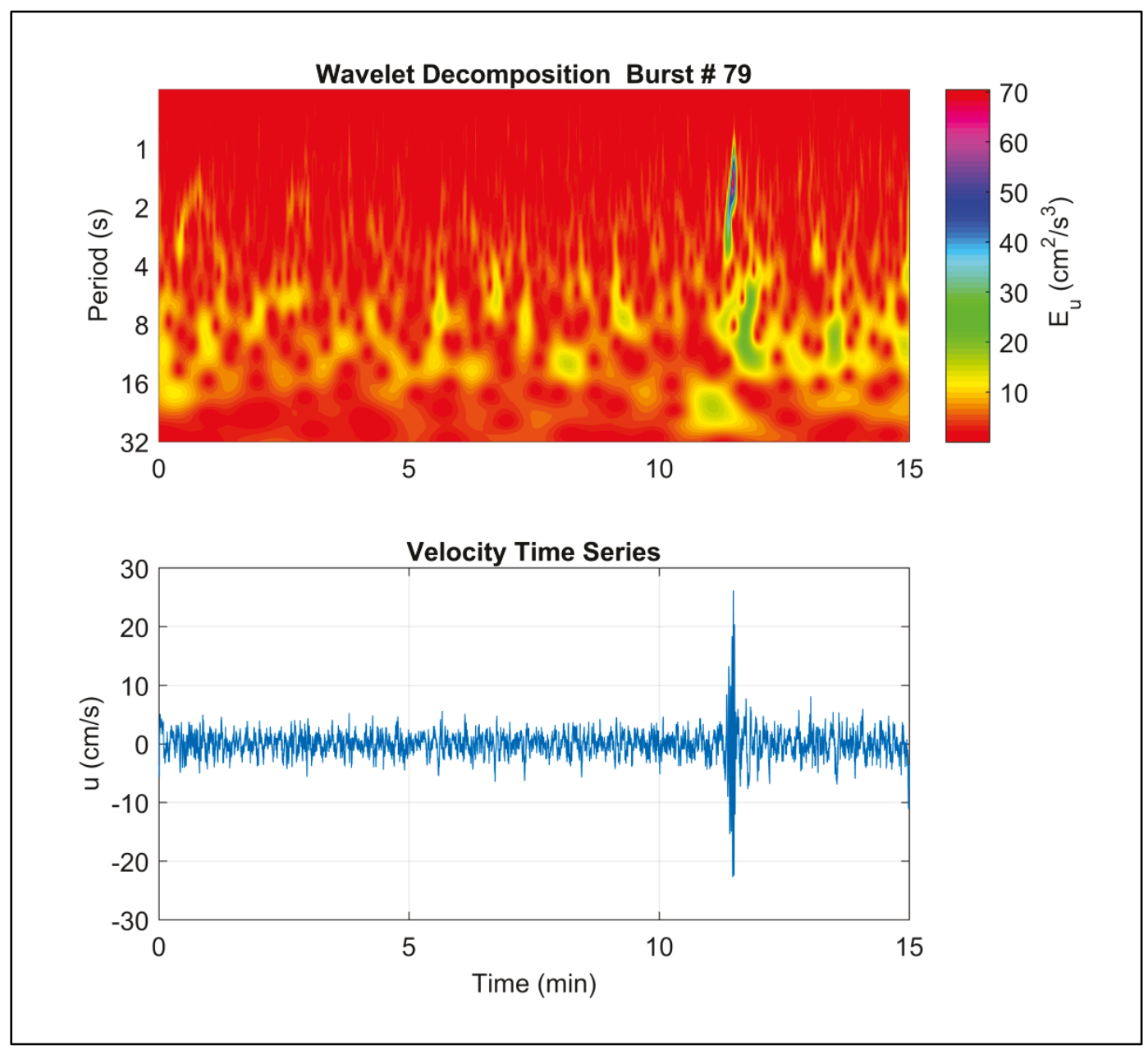


Figure C.9. Wavelet analysis for the ninth burst with vessels. This is the last burst with vessels. Mean along channel currents are the highest measured for the study, which is reflected in the wavelet analysis as lower-frequency energy density pockets and larger fluctuations in the time series.

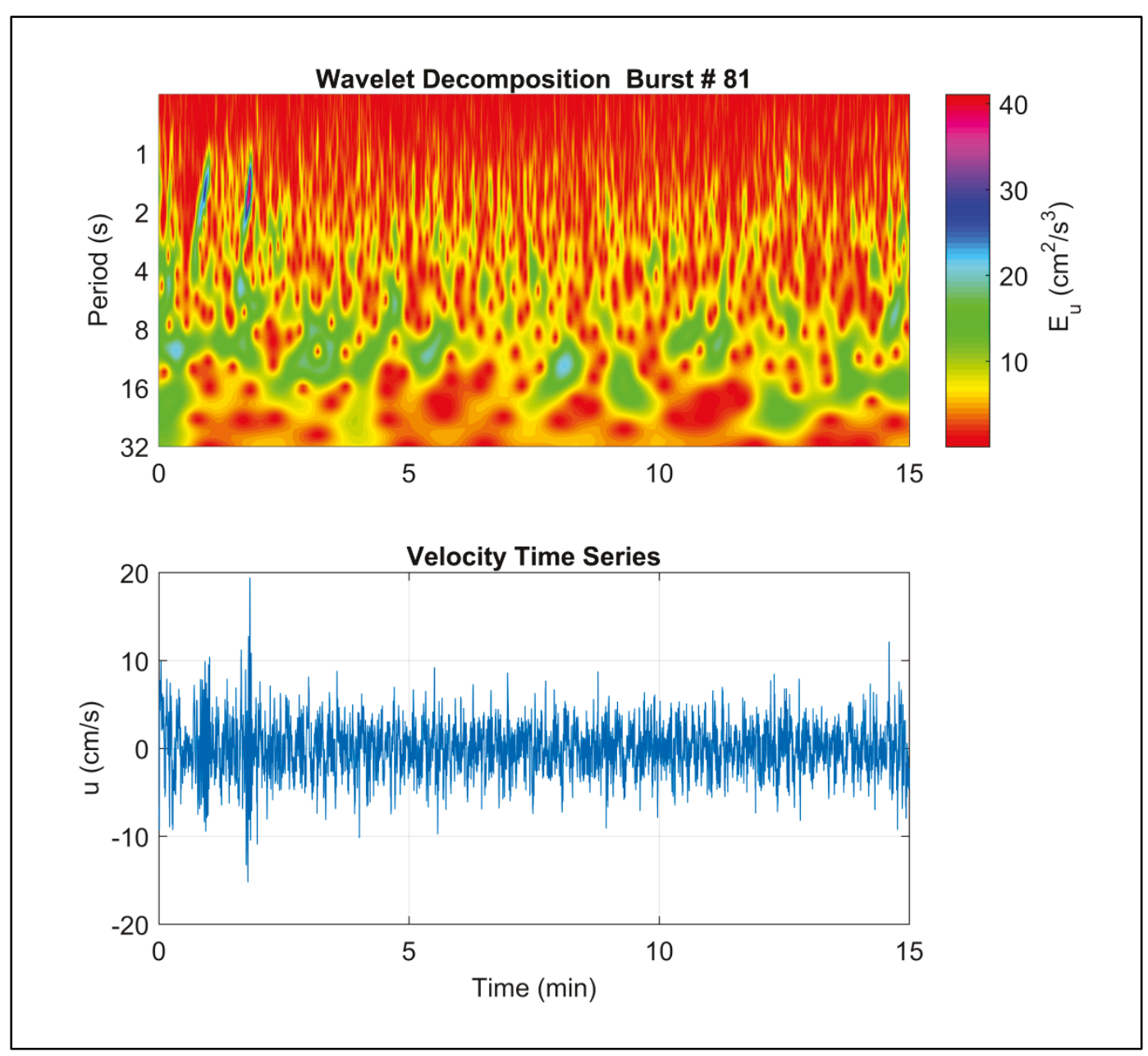




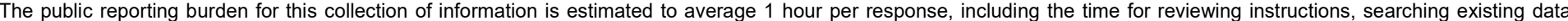

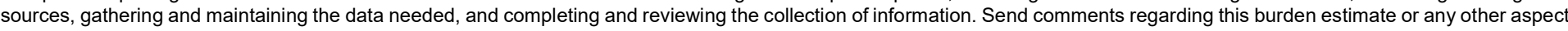

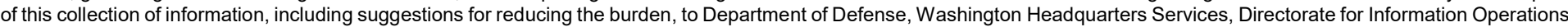

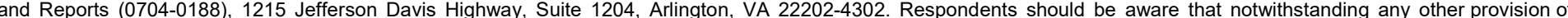
law, no person shall be subject to any penalty for failing to comply with a collection of information if it does not display a currently valid OMB control number.

PLEASE DO NOT RETURN YOUR FORM TO THE ABOVE ADDRESS.

\begin{tabular}{l|l|l}
$\begin{array}{l}\text { 1. REPORT DATE } \\
\text { May } 2018\end{array}$ & $\begin{array}{l}\text { 2. REPORT TYPE } \\
\text { Final Report }\end{array}$ & 3. DATES COVERED (From - To) \\
\hline
\end{tabular}

\section{TITLE AND SUBTITLE}

Final Report

Wave Characteristics and Sediment Resuspension by Recreational Vessels in Coastal Plain

Saltmarshes

5a. CONTRACT NUMBER

5b. GRANT NUMBER

5c. PROGRAM ELEMENT NUMBER

6. $\operatorname{AUTHOR(S)}$

Richard Styles and Michael A. Hartman

5d. PROJECT NUMBER

5e. TASK NUMBER

5f. WORK UNIT NUMBER

L85290

7. PERFORMING ORGANIZATION NAME(S) AND ADDRESS(ES) (see reverse)

Coastal and Hydraulics Laboratory

U.S. Army Engineer Research and Development Center

3909 Halls Ferry Road

8. PERFORMING ORGANIZATION REPORT NUMBER

Vicksburg MS 39180

ERDC/CHL TR-18-5

9. SPONSORING/MONITORING AGENCY NAME(S) AND ADDRESS(ES)

U.S. Army Corps of Engineers

Washington, DC 20314-1000

10. SPONSOR/MONITOR'S ACRONYM(S)

HQUSACE

\section{SPONSOR/MONITOR'S REPORT} NUMBER(S)

\section{DISTRIBUTION/AVAILABILITY STATEMENT}

Approved for public release; distribution is unlimited.

\section{SUPPLEMENTARY NOTES}

\section{ABSTRACT}

The characteristics of vessel-generated waves can form an integral component of navigation studies in coastal and inland waterways. In vulnerable areas, such as coastal wetlands, vessel wake can be a primary cause of shoreline erosion. While a few studies have investigated the role of commercial vessels operating in navigation channels adjacent to coastal marshes, little has been done regarding the role of recreational craft operating within the marsh interior. To gain further insight into the characteristics of vessel-generated waves, this study measured flow, turbulence, wave-generated currents, suspended sediment concentration, and particle size distribution in an inter-tidal salt marsh with significant tidal influence. The results are used to develop empirical equations of sediment concentration and settling velocity and to apply these equations to an existing vessel wake model. The utility of the model is demonstrated by calculating the energy dissipation due to vessels and comparing the result to the equivalent tidal energy. The cumulative energy dissipation due to vessels reveals how this information can be used to help manage recreational vessel traffic in tide-dominated coastal plain saltmarshes and extend the results of this study to a wider range of forcing conditions.

\section{SUBJECT TERMS}

Boats and boating_Environmental aspects, Hydrodynamics, Salt marshes_-Erosion, Suspended sediments, Water waves

16. SECURITY CLASSIFICATION OF:

a. REPORT

Unclassified

\section{b. ABSTRACT}

Unclassified

\section{c. THIS PAGE}

Unclassified
17. LIMITATION OF ABSTRACT

SAR
18. NUMBER OF PAGES

87 19a. NAME OF RESPONSIBLE PERSON

Richard Styles

19b. TELEPHONE NUMBER (Include area code) 601-634-4065 